\title{
Spatial decay of rotating waves in reaction diffusion systems
}

\author{
Wolf-Jürgen Beyn and Denny Otten
}

Communicated by Y. Charles Li, received February 17, 2016.

ABSTRACT. In this paper we study nonlinear problems for Ornstein-Uhlenbeck operators

$$
A \triangle v(x)+\langle S x, \nabla v(x)\rangle+f(v(x))=0, x \in \mathbb{R}^{d}, d \geqslant 2,
$$

where the matrix $A \in \mathbb{R}^{N, N}$ is diagonalizable and has eigenvalues with positive real part, the map $f: \mathbb{R}^{N} \rightarrow \mathbb{R}^{N}$ is sufficiently smooth and the matrix $S \in \mathbb{R}^{d, d}$ in the unbounded drift term is skew-symmetric. Nonlinear problems of this form appear as stationary equations for rotating waves in time-dependent reaction diffusion systems. We prove under appropriate conditions that every bounded classical solution $v_{\star}$ of the nonlinear problem, which falls below a certain threshold at infinity, already decays exponentially in space, in the sense that $v_{\star}$ belongs to an exponentially weighted Sobolev space $W_{\theta}^{1, p}\left(\mathbb{R}^{d}, \mathbb{R}^{N}\right)$. Several extensions of this basic result are presented: to complex-valued systems, to exponential decay in higher order Sobolev spaces and to pointwise estimates. We also prove that every bounded classical solution $v$ of the eigenvalue problem

$$
A \triangle v(x)+\langle S x, \nabla v(x)\rangle+D f\left(v_{\star}(x)\right) v(x)=\lambda v(x), x \in \mathbb{R}^{d}, d \geqslant 2,
$$

decays exponentially in space, provided $\operatorname{Re} \lambda$ lies to the right of the essential spectrum. As an application we analyze spinning soliton solutions which occur in the Ginzburg-Landau equation. Our results form the basis for investigating nonlinear stability of rotating waves in higher space dimensions and truncations to bounded domains.

\section{Contents}

1. Introduction

2. Assumptions and main result

3. Variable coefficient complex Ornstein-Uhlenbeck operators 204

4. Exponential decay of rotating nonlinear waves 214

5. Exponential decay of eigenfunctions

1991 Mathematics Subject Classification. Primary 35K57; Secondary 35B40, 47A55, 35Pxx, 35Q56, 47N40.

Key words and phrases. Rotating waves, spatial exponential decay, Ornstein-Uhlenbeck operator, exponentially weighted resolvent estimates, reaction-diffusion equations. 
6. Rotating waves in reaction diffusion systems:

The cubic-quintic complex Ginzburg-Landau equation

\section{Introduction}

In the present paper we study systems of reaction-diffusion equations

$$
\begin{aligned}
u_{t}(x, t) & =A \triangle u(x, t)+f(u(x, t)), t>0, x \in \mathbb{R}^{d}, d \geqslant 2, \\
u(x, 0) & =u_{0}(x) \quad, t=0, x \in \mathbb{R}^{d},
\end{aligned}
$$

where $A \in \mathbb{R}^{N, N}$ is a diffusion matrix, $f: \mathbb{R}^{N} \rightarrow \mathbb{R}^{N}$ is a sufficiently smooth nonlinearity, $u_{0}: \mathbb{R}^{d} \rightarrow \mathbb{R}^{N}$ are the initial data and $u: \mathbb{R}^{d} \times[0, \infty) \rightarrow \mathbb{R}^{N}$ denotes a vector-valued solution.

We are mainly interested in rotating wave solutions of (1.1) which are of the form

$$
u_{\star}(x, t)=v_{\star}\left(e^{-t S} x\right), t \geqslant 0, x \in \mathbb{R}^{d}, d \geqslant 2
$$

with space-dependent profile $v_{\star}: \mathbb{R}^{d} \rightarrow \mathbb{R}^{N}$ and skew-symmetric matrix $S \in \mathbb{R}^{d, d}$. The skew-symmetry of $S$ implies that $e^{-t S}$ describes a rotation in $\mathbb{R}^{d}$, and hence $u_{\star}$ is a solution rotating at constant velocity while maintaining its shape determined by $v_{\star}$. The profile $v_{\star}$ is called (exponentially) localized, if it tends (exponentially) to some constant vector $v_{\infty} \in \mathbb{R}^{N}$ as $|x| \rightarrow \infty$.

Transforming (1.1) via $u(x, t)=v\left(e^{-t S} x, t\right)$ into a co-rotating frame yields the evolution equation

$$
\begin{aligned}
& v_{t}(x, t)=A \triangle v(x, t)+\langle S x, \nabla v(x, t)\rangle+f(v(x, t)), t>0, x \in \mathbb{R}^{d}, d \geqslant 2, \\
& v(x, 0)=u_{0}(x) \quad, t=0, x \in \mathbb{R}^{d} .
\end{aligned}
$$

The diffusion and drift term are given by

$$
A \triangle v(x):=A \sum_{i=1}^{d} \frac{\partial^{2}}{\partial x_{i}^{2}} v(x) \quad \text { and } \quad\langle S x, \nabla v(x)\rangle:=\sum_{i=1}^{d} \sum_{j=1}^{d} S_{i j} x_{j} D_{i} v(x) .
$$

The pattern $v_{\star}$ itself appears as a stationary solution of (1.3), i.e. $v_{\star}$ solves the steady state problem

$$
A \triangle v_{\star}(x)+\left\langle S x, \nabla v_{\star}(x)\right\rangle+f\left(v_{\star}(x)\right)=0, x \in \mathbb{R}^{d}, d \geqslant 2 .
$$

We may write $(1.5)$ as $\left[\mathcal{L}_{0} v_{\star}\right](x)+f\left(v_{\star}(x)\right)=0$ by introducing the OrnsteinUhlenbeck operator

$$
\left[\mathcal{L}_{0} v\right](x):=A \triangle v(x)+\langle S x, \nabla v(x)\rangle, x \in \mathbb{R}^{d} .
$$

By the skew-symmetry of $S$ we can write the drift term in terms of angular derivatives as follows

$$
\langle S x, \nabla v(x)\rangle=\sum_{i=1}^{d-1} \sum_{j=i+1}^{d} S_{i j}\left(x_{j} \frac{\partial}{\partial x_{i}}-x_{i} \frac{\partial}{\partial x_{j}}\right) v(x) .
$$

The aim of this paper is to derive suitable conditions guaranteeing that every localized rotating wave of (1.1) is already exponentially localized. More precisely, the main theorem states the following: if the difference $v_{\star}-v_{\infty}$ of a rotating wave 
to its far field value falls below a certain threshold at infinity, then it decays exponentially in space. The decay is specified by showing that $v_{\star}-v_{\infty}$ belongs to some exponentially weighted Sobolev space $W_{\theta}^{1, p}\left(\mathbb{R}^{d}, \mathbb{R}^{N}\right), 1<p<\infty$. Our key assumption requires all eigenvalues of the Jacobian $D f\left(v_{\infty}\right)$ to have negative real part.

We extend this result to complex-valued systems and then apply it to prove exponential decay of localized spinning solitons arising in the cubic-quintic complex Ginzburg-Landau equation (QCGL), [14]. Figure 1(a) shows the real part of a spinning soliton $v_{\star}$ in two space dimensions, while Figure 1(b) shows the isosurfaces of the real part of a spinning soliton in three space dimensions. Both of these rotating waves are exponentially localized, as our results will show. Two nonlocalized rotating waves are illustrated in Figure 1(c)-(d). Figure 1(c) shows the real part of a spiral wave in two space dimensions and Figure 1(d) the isosurfaces of the real part of an untwisted scroll wave. In Section 6 below we will discuss this example in more detail.

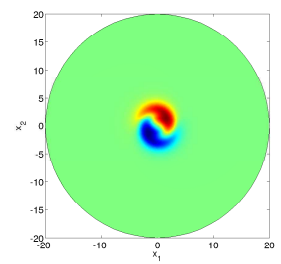

(a)

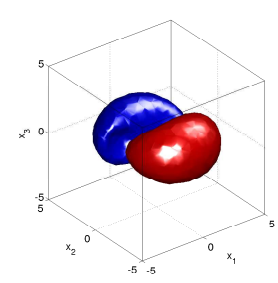

(b)

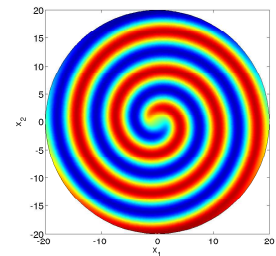

(c)

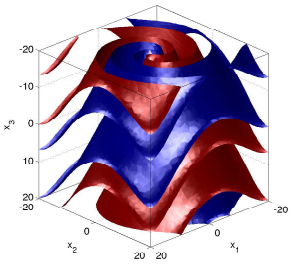

(d)

Figure 1. Rotating waves of QCGL (6.1). (a) Spinning solitons for $d=2$ with colorbar reaching from -1.6 (blue) to 1.6 (red), (b) spinning soliton for $d=3$ with isosurfaces at values -0.5 (blue) and 0.5 (red), (c) spiral wave for $d=2$ with colorbar reaching from -1.7 (blue) to 1.7 (red), and (d) scroll wave for $d=3$ with isosurfaces at values -0.5 (blue) and 0.5 (red)

An important issue is to investigate nonlinear stability of rotating waves (more precisely, stability with asymptotic phase) in reaction diffusion systems, see [5]. A well known task is to derive nonlinear stability from linear stability of the linearized operator

$$
[\mathcal{L} v](x):=\left[\mathcal{L}_{0} v\right](x)+D f\left(v_{\star}(x)\right) v(x), x \in \mathbb{R}^{d} .
$$

By linear stability (also called strong spectral stability) we mean that the essential spectrum and the isolated eigenvalues of $\mathcal{L}$ lie strictly to the left of the imaginary axis, except for those on the imaginary axis caused by Euclidean equivariance, [26, Ch.9]. This requires to study isolated eigenvalues $\lambda \in \mathbb{C}$ of the problem

$$
[(\lambda I-\mathcal{L}) v](x)=0, x \in \mathbb{R}^{d} .
$$

A further aim of this paper is to prove that every bounded eigenfunction $v$ of the linearized operator $\mathcal{L}$ decays exponentially in space, provided the real parts of the associated (isolated) eigenvalues $\lambda$ lie to the right of the essential spectrum. To be more precise, we show that for such values of $\lambda$, every bounded classical solution $v$ of the eigenvalue problem (1.9) belongs to some exponentially weighted 
Sobolev space $W_{\theta}^{1, p}\left(\mathbb{R}^{d}, \mathbb{R}^{N}\right)$ for some $1<p<\infty$. In particular, we prove that the eigenfunction $v(x)=\left\langle S x, \nabla v_{\star}(x)\right\rangle$ associated to the eigenvalue $\lambda=0$ decays exponentially in space.

A nonlinear stability result for two dimensional localized rotating patterns was proved by Beyn and Lorenz in [5]. Their proof requires three essential assumptions: The matrix $D f\left(v_{\infty}\right)$ is stable, meaning that all its eigenvalues have a negative real part. Moreover, strong spectral stability in the sense above is assumed. And finally, the profile $v_{\star}$ of the rotating wave and its derivatives up to order 2 decay to zero at infinity. Their analysis shows that the decay of the rotating wave itself and the spectrum of the linearization are both crucial for investigating nonlinear stability. A corresponding result on nonlinear stability of nonlocalized rotating waves, such as spiral waves and scroll waves, is still an open problem. The difficulty is related to the fact that the essential spectrum touches the imaginary axis at infinitely many points. The spectrum of the linearization at (nonlocalized) spiral waves is well-known and has been extensively studied by Sandstede, Scheel and Fiedler in $[16,31,32]$.

For numerical computations it is essential to truncate the equations (1.1), (1.3) and (1.9) to a bounded domain, so that standard approximations, e.g. with finite elements, apply. The truncation error arising in this process, depends on the boundary conditions. Assuming that a rotating wave is (exponentially) localized, we can expect the truncation error to be (exponentially) small as well. For this reason, the exponential decay of rotating waves plays a fundamental role when estimating errors caused by approximations of rotating waves on bounded domains.

We consider our results on the decay of rotating waves for (1.1) on the whole $\mathbb{R}^{d}$ as a first step in studying such truncation errors. Despite numerous numerical simulations of spiral behavior on bounded domains, a rigorous analysis of the errors caused by spatial truncation seems not to be available.

We emphasize that the results from Section 3-6 are extensions of the results from the $\mathrm{PhD}$ thesis [26]. One major improvement refers to the fact that our main result Theorem 2.8 avoids the additional assumption $v_{\star} \in L^{p}\left(\mathbb{R}^{d}, \mathbb{R}^{N}\right)$ from $[26$, Thm.1.8] by using ideas from the work [6].

\section{Assumptions and main result}

2.1. Assumptions and main result. Consider the steady state problem

$$
A \triangle v(x)+\langle S x, \nabla v(x)\rangle+f(v(x))=0, x \in \mathbb{R}^{d}, d \geqslant 2,
$$

with diffusion matrix $A \in \mathbb{K}^{N, N}$ and a function $f: \mathbb{K}^{N} \rightarrow \mathbb{K}^{N}$ for $\mathbb{K} \in\{\mathbb{R}, \mathbb{C}\}$. Recall the Ornstein-Uhlenbeck operator from (1.6) with drift and diffusion term specified in (1.4).

We define a rotating wave $u_{\star}$ as follows:

Definition 2.1. A function $u_{\star}: \mathbb{R}^{d} \times[0, \infty) \rightarrow \mathbb{K}^{N}$ is called a rotating wave (or rotating pattern) if it has the form

$$
u_{\star}(x, t)=v_{\star}\left(e^{-t S}\left(x-x_{\star}\right)\right), x \in \mathbb{R}^{d}, t \in[0, \infty),
$$

with profile (or pattern) $v_{\star}: \mathbb{R}^{d} \rightarrow \mathbb{K}^{N}$, a skew-symmetric matrix $0 \neq S \in \mathbb{R}^{d, d}$ and $x_{\star} \in \mathbb{R}^{d}$. A rotating wave $u_{\star}$ is called localized (exponentially localized with decay 
rate $\eta$ ) if it satisfies

$$
\lim _{|x| \rightarrow \infty} e^{\eta|x|}\left|v_{\star}(x)-v_{\infty}\right|=0 \text { for some } v_{\infty} \in \mathbb{K}^{N}
$$

and for $\eta=0(\eta>0)$. It is called nonlocalized, if it is not localized in the sense above.

The vector $x_{\star} \in \mathbb{R}^{d}$ can be considered as the center of rotation for $d=2$ and as the support vector of the axis of rotation for $d=3$. In case $d \in\{2,3\}, S$ can be considered as the angular velocity tensor associated to the angular velocity vector $\omega \in \mathbb{R}^{\frac{d(d-1)}{2}}$ containing $S_{i j}, i=1, \ldots, d-1, j=i+1, \ldots, d$. Some examples of rotating patterns are illustrated in Figure 1 and will be treated in Section 6 below.

In the following we will impose various restrictions on the matrix $A$ :

Assumption 2.2. For $A \in \mathbb{K}^{N, N}$ with $\mathbb{K} \in\{\mathbb{R}, \mathbb{C}\}$ and $1<p<\infty$ consider the conditions

(A1) $A$ is diagonalizable (over $\mathbb{C}$ ),

(A2) $\operatorname{Re} \sigma(A)>0$,

(A3) $\operatorname{Re}\langle w, A w\rangle \geqslant \beta_{A} \forall w \in \mathbb{K}^{N},|w|=1$ for some $\beta_{A}>0$,

(A4) There exists $\gamma_{A}>0$ such that for all $z, w \in \mathbb{K}^{N}$

$$
|z|^{2} \operatorname{Re}\langle w, A w\rangle+(p-2) \operatorname{Re}\langle w, z\rangle \operatorname{Re}\langle z, A w\rangle \geqslant \gamma_{A}|z|^{2}|w|^{2},
$$

(A5) $A$ is invertible and $\mu_{1}(A)>\frac{|p-2|}{p}$,

(to be read as $A=a>0$ in case $N=1, \mathbb{K}=\mathbb{R}$ ).

Assumption (A1) is a system condition and ensures that all results for scalar equations can be extended to system cases. This condition is independent of (A2)(A5) and is used in $[26,27]$ to derive an explicit formula for the heat kernel of $\mathcal{L}_{0}$. A typical case where (A1) holds, is a scalar complex-valued equation when transformed into a real-valued system of dimension 2 . The positivity condition (A2) guarantees that the diffusion part $A \triangle$ is an elliptic operator. All eigenvalues $\lambda \in \sigma(A)$ of $A$ lie in the open right half-plane $\{\lambda \in \mathbb{C} \mid \operatorname{Re} \lambda>0\}$. Condition (A2) guarantees that $A^{-1}$ exists and that $-A$ is a stable matrix. The strict accretivity condition (A3) is more restrictive than (A2). In (A3) we use $\langle u, v\rangle:=\bar{u}^{T} v$ to denote the standard inner product on $\mathbb{K}^{N}$. Recall that condition (A2) is satisfied iff there exists an inner product $[\cdot, \cdot]$ and some $\beta_{A}>0$ such that $\operatorname{Re}[w, A w] \geqslant \beta_{A}$ forall $w \in \mathbb{K}^{N}$ with $[w, w]=1$. Condition (A3) ensures that the differential operator $\mathcal{L}_{0}$ is closed on its (local) domain $\mathcal{D}_{\text {loc }}^{p}\left(\mathcal{L}_{0}\right)$, see Theorem 2.12 below. The $L^{p}$-dissipativity condition (A4) is more restrictive than (A3) and imposes additional requirements on the spectrum of $A$. This condition, which comes originally from $[\mathbf{1 1}, \mathbf{1 2}]$, is used in $[26,29]$ to prove $L^{p}$-resolvent estimates for $\mathcal{L}_{0}$. A geometrical meaning of (A4) can be given in terms of the antieigenvalues of the diffusion matrix $A$. In $[26,28]$, it is proved that condition (A4) is equivalent to the $L^{p}$-antieigenvalue condition (A5). Condition (A5) requires that the first antieigenvalue of $A$ (see $[17,18]$ ), defined by

$$
\mu_{1}(A):=\inf _{\substack{w \in \mathbb{K}^{N} \\ w \neq 0 \\ A w \neq 0}} \frac{\operatorname{Re}\langle w, A w\rangle}{|w||A w|}=\inf _{\substack{w \in \mathbb{K}^{N} \\|w|=1 \\ A w \neq 0}} \frac{\operatorname{Re}\langle w, A w\rangle}{|A w|},
$$


is bounded from below by a non-negative $p$-dependent constant. Condition (A5) is also equivalent to the following $p$-dependent upper bound for the (real) angle of $A$ (cf. [17]),

$$
\Phi_{\mathbb{R}}(A):=\cos ^{-1}\left(\mu_{1}(A)\right)<\cos ^{-1}\left(\frac{|p-2|}{p}\right) \in\left(0, \frac{\pi}{2}\right], \quad 1<p<\infty .
$$

Therefore, the first antieigenvalue $\mu_{1}(A)$ can be considered as the cosine of the maximal (real) turning angle of vectors mapped by the matrix $A$. Some special cases in which the first antieigenvalue can be given explicitly are treated in [28]. We summarize the relationship of (A2)-(A5):

$$
A \text { invertible } \Longleftarrow(\mathrm{A} 2) \Longleftarrow(\mathrm{A} 3) \Longleftarrow(\mathrm{A} 4) \Longleftarrow(\mathrm{A} 5) \text {. }
$$

We continue with the rotational condition (A6) and a smoothness condition (A7),

Assumption 2.3. The matrix $S \in \mathbb{R}^{d, d}$ satisfies

(A6) $S$ is skew-symmetric, i.e. $S=-S^{T}$.

Assumption 2.4. The function $f: \mathbb{R}^{N} \rightarrow \mathbb{R}^{N}$ satisfies (A7) $f \in C^{2}\left(\mathbb{R}^{N}, \mathbb{R}^{N}\right)$.

Later on we apply our results to complex-valued nonlinearities of the form

$$
f: \mathbb{C}^{N} \rightarrow \mathbb{C}^{N}, \quad f(u)=g\left(|u|^{2}\right) u,
$$

where $g: \mathbb{R} \rightarrow \mathbb{C}^{N, N}$ is a sufficiently smooth function. Such nonlinearities arise for example in Ginzburg-Landau equations, Schrödinger equations, $\lambda-\omega$ systems and many other equations from physical sciences, see Section 6 . Note, that in this case, the function $f$ is not holomorphic in $\mathbb{C}$, but its real-valued version in $\mathbb{R}^{2}$ satisfies (A7) if $g \in C^{2}$. For differentiable functions $f: \mathbb{R}^{N} \rightarrow \mathbb{R}^{N}$ we denote by $D f$ the Jacobian matrix in the real sense.

Assumption 2.5. For $v_{\infty} \in \mathbb{R}^{N}$ consider the following conditions:

(A8) $f\left(v_{\infty}\right)=0$,

(A9) $A, D f\left(v_{\infty}\right) \in \mathbb{R}^{N, N}$ are simultaneously diagonalizable (over $\mathbb{C}$ ),

$(\mathrm{A} 10) \operatorname{Re} \sigma\left(D f\left(v_{\infty}\right)\right)<0$,

(A11) There exists $\beta_{\infty}>0$ such that for all $w \in \mathbb{K}^{N}$ with $|w|=1$

$$
\operatorname{Re}\left\langle w,-D f\left(v_{\infty}\right) w\right\rangle \geqslant \beta_{\infty} .
$$

The constant asymptotic state condition (A8) requires $v_{\infty}$ to be a steady state of the nonlinear equation. The system condition (A9) is an extension of Assumption (A1), and the coercivity condition (A11) is again more restrictive than the spectral condition (A10).

Definition 2.6. A function $v_{\star}: \mathbb{R}^{d} \rightarrow \mathbb{K}^{N}$ is called a classical solution of $(2.1)$ if

$$
v_{\star} \in C^{2}\left(\mathbb{R}^{d}, \mathbb{K}^{N}\right)
$$

and $v_{\star}$ solves (2.1) pointwise. 
Later on, we will consider classical solutions $v_{\star}$ which are even bounded, i.e. $v_{\star} \in C_{\mathrm{b}}\left(\mathbb{R}^{d}, \mathbb{K}^{N}\right)$. For matrices $C \in \mathbb{K}^{N, N}$ with spectrum $\sigma(C)$ we denote by $\rho(C):=\max _{\lambda \in \sigma(C)}|\lambda|$ its spectral radius and by $s(C):=\max _{\lambda \in \sigma(C)} \operatorname{Re} \lambda$ its spectral abscissa (or spectral bound). With this notation, we define the following constants which appear in the linear theory from $[26,27,29]$ :

$$
\begin{aligned}
a_{\min } & :=\left(\rho\left(A^{-1}\right)\right)^{-1}, \quad a_{\max }:=\rho(A), \quad a_{0}:=-s(-A), \\
a_{1} & :=\left(\frac{a_{\max }^{2}}{a_{\min } a_{0}}\right)^{\frac{d}{2}}, \quad b_{0}:=-s\left(D f\left(v_{\infty}\right)\right) .
\end{aligned}
$$

Recall the relations $0<a_{0} \leq \beta_{A}$ and $0<b_{0} \leq \beta_{\infty}$ to the coercivity constants from (A3),(A11). Our main tool for investigating exponential decay in space are exponentially weighted function spaces. For the choice of weight function we follow [40, Def.3.1]:

Definition 2.7. (1) A function $\theta \in C\left(\mathbb{R}^{d}, \mathbb{R}\right)$ is called a weight function of exponential growth rate $\eta \geqslant 0$ provided that

(W1) $\theta(x)>0 \forall x \in \mathbb{R}^{d}$,

(W2) $\exists C_{\theta}>0: \theta(x+y) \leqslant C_{\theta} \theta(x) e^{\eta|y|} \forall x, y \in \mathbb{R}^{d}$.

(2) A weight function $\theta \in C\left(\mathbb{R}^{d}, \mathbb{R}\right)$ of exponential growth rate $\eta \geqslant 0$ is called radial if

(W3) $\exists \phi:[0, \infty) \rightarrow \mathbb{R}: \theta(x)=\phi(|x|) \forall x \in \mathbb{R}^{d}$.

(3) A radial weight function $\theta \in C\left(\mathbb{R}^{d}, \mathbb{R}\right)$ of exponential growth rate $\eta \geqslant 0$ is called nondecreasing (or monotonically increasing) provided that

(W4) $\theta(x) \leqslant \theta(y) \forall x, y \in \mathbb{R}^{d}$ with $|x| \leqslant|y|$.

Standard examples of radial weight functions are

$$
\theta_{1}(x)=\exp (\mu|x|) \quad \text { and } \quad \theta_{2}(x)=\cosh (\mu|x|),
$$

as well as their smooth analogs

$$
\theta_{3}(x)=\exp \left(\mu \sqrt{|x|^{2}+1}\right) \quad \text { and } \quad \theta_{4}(x)=\cosh \left(\mu \sqrt{|x|^{2}+1}\right)
$$

for $x \in \mathbb{R}^{d}$ and $\mu \in \mathbb{R}$. Obviously, all these functions are radial weight functions of exponential growth rate $\eta=|\mu|$ with $C_{\theta}=1$. Moreover, $\theta_{2}, \theta_{4}$ are nondecreasing for any $\mu \in \mathbb{R}$ and $\theta_{1}, \theta_{3}$ if $\mu \geqslant 0$.

With every weight function of exponential growth rate we associate exponentially weighted Lebesgue and Sobolev spaces

$$
\begin{aligned}
L_{\theta}^{p}\left(\mathbb{R}^{d}, \mathbb{K}^{N}\right) & :=\left\{u \in L_{\mathrm{loc}}^{1}\left(\mathbb{R}^{d}, \mathbb{K}^{N}\right) \mid\|\theta u\|_{L^{p}}<\infty\right\}, \\
W_{\theta}^{k, p}\left(\mathbb{R}^{d}, \mathbb{K}^{N}\right) & :=\left\{u \in L_{\theta}^{p}\left(\mathbb{R}^{d}, \mathbb{K}^{N}\right)\left|D^{\beta} u \in L_{\theta}^{p}\left(\mathbb{R}^{d}, \mathbb{K}^{N}\right) \forall\right| \beta \mid \leqslant k\right\},
\end{aligned}
$$

for every $1 \leqslant p \leqslant \infty$ and $k \in \mathbb{N}_{0}$.

With these preparations we can formulate the main result of our paper.

THEOREM 2.8 (Exponential decay of $v_{\star}$ ). Let the assumptions (A4), (A6)-(A9) and (A11) be satisfied for $\mathbb{K}=\mathbb{R}$ and for some $1<p<\infty$. Moreover, let $a_{\max }=\rho(A)$ denote the spectral radius of $A,-a_{0}=s(-A)$ the spectral bound of $-A$ and $-b_{0}=s\left(D f\left(v_{\infty}\right)\right)$ the spectral bound of $D f\left(v_{\infty}\right)$. Further, let 
$\theta(x)=\exp \left(\mu \sqrt{|x|^{2}+1}\right)$ denote a weight function for $\mu \in \mathbb{R}$. Then, for every $0<\varepsilon<1$ there is a constant $K_{1}=K_{1}\left(A, f, v_{\infty}, d, p, \varepsilon\right)>0$ with the following property: Every classical solution $v_{\star}$ of

$$
A \triangle v(x)+\langle S x, \nabla v(x)\rangle+f(v(x))=0, x \in \mathbb{R}^{d},
$$

such that

$$
\sup _{|x| \geqslant R_{0}}\left|v_{\star}(x)-v_{\infty}\right| \leqslant K_{1} \text { for some } R_{0}>0
$$

satisfies

$$
v_{\star}-v_{\infty} \in W_{\theta}^{1, p}\left(\mathbb{R}^{d}, \mathbb{R}^{N}\right)
$$

for every exponential decay rate

$$
0 \leqslant \mu \leqslant \varepsilon \frac{\sqrt{a_{0} b_{0}}}{a_{\max } p} .
$$

Roughly speaking, Theorem 2.8 states that every bounded classical solution $v_{\star}$ which is sufficiently close to the steady state $v_{\infty}$ at infinity, see (2.9), must decay exponentially in space. The exponential decay is expressed by the fact, that $v_{\star}-v_{\infty}$ belongs to an exponentially weighted Sobolev space. Moreover, the theorem gives an explicit bound for the exponential growth rate, that depends only on $p$, the spectral radius of $A$, and the spectral abscissas of $-A$ and $D f\left(v_{\infty}\right)$. The role of $\varepsilon$ becomes clear upon noting that $K_{1} \rightarrow 0$ as $\varepsilon \rightarrow 1$ whereas $K_{1} \rightarrow K_{1}^{0}>0$ as $\varepsilon \rightarrow 0$. The stronger the exponential rate, the closer the solution $v_{\star}$ has to approach $v_{\infty}$ at infinity.

\subsection{Outline of proof: Decomposition of linear differential operators.} In the following we explain the decomposition of differential operators that leads to the proof of Theorem 2.8 .

Far-Field Linearization. Consider the nonlinear problem

$$
A \triangle v_{\star}(x)+\left\langle S x, \nabla v_{\star}(x)\right\rangle+f\left(v_{\star}(x)\right)=0, x \in \mathbb{R}^{d}, d \geqslant 2 .
$$

Let $v_{\infty} \in \mathbb{R}^{N}$ be the constant asymptotic state satisfying (A8) and let $f \in C^{1}\left(\mathbb{R}^{N}, \mathbb{R}^{N}\right)$. By the Mean Value Theorem we can write

$$
f\left(v_{\star}(x)\right)=\underbrace{f\left(v_{\infty}\right)}_{=0}+\underbrace{\int_{0}^{1} D f\left(v_{\infty}+t\left(v_{\star}(x)-v_{\infty}\right)\right) d t}_{=: a(x)}\left(v_{\star}(x)-v_{\infty}\right), x \in \mathbb{R}^{d} .
$$

From $v_{\star} \in C_{\mathrm{b}}\left(\mathbb{R}^{d}, \mathbb{R}^{N}\right)$ we deduce $a \in C_{\mathrm{b}}\left(\mathbb{R}^{d}, \mathbb{R}^{N, N}\right)$. Moreover, since the classical solution $v_{\star}$ solves (2.11) pointwise and $v_{\infty} \in \mathbb{R}^{N}$ is constant, the difference $w_{\star}:=$ $v_{\star}-v_{\infty}$ belongs to $C^{2}\left(\mathbb{R}^{d}, \mathbb{R}^{N}\right) \cap C_{\mathrm{b}}\left(\mathbb{R}^{d}, \mathbb{R}^{N}\right)$ and satisfies the linearized equation

$$
\left[\mathcal{L} w_{\star}\right](x)=A \triangle w_{\star}(x)+\left\langle S x, \nabla w_{\star}(x)\right\rangle+a(x) w_{\star}(x)=0, x \in \mathbb{R}^{d} .
$$

In order to study the behavior of solutions to (2.11) as $|x| \rightarrow \infty$, we decompose the variable coefficient $a(x)$ in (2.12).

Decomposition of $a$. Let $a(x)=D f\left(v_{\infty}\right)+Q(x)$ with $Q$ defined by

$$
Q(x):=\int_{0}^{1} D f\left(v_{\infty}+t w_{\star}(x)\right)-D f\left(v_{\infty}\right) d t, x \in \mathbb{R}^{d} .
$$


This yields $Q \in C_{\mathrm{b}}\left(\mathbb{R}^{d}, \mathbb{R}^{N, N}\right)$ and (2.12) reads as

$$
A \triangle w_{\star}(x)+\left\langle S x, \nabla w_{\star}(x)\right\rangle+\left(D f\left(v_{\infty}\right)+Q(x)\right) w_{\star}(x)=0, x \in \mathbb{R}^{d} .
$$

Decomposition of $Q$. Let $Q(x)=Q_{\mathrm{s}}(x)+Q_{\mathrm{c}}(x)$, where $Q_{\mathrm{s}} \in C_{\mathrm{b}}\left(\mathbb{R}^{d}, \mathbb{R}^{N, N}\right)$ is small w.r.t. $\|\cdot\|_{\infty}$ and $Q_{\mathrm{c}} \in C_{\mathrm{b}}\left(\mathbb{R}^{d}, \mathbb{R}^{N, N}\right)$ is compactly supported on $\mathbb{R}^{d}$, see Figure 2. Then, we arrive at

$$
A \triangle w_{\star}(x)+\left\langle S x, \nabla w_{\star}(x)\right\rangle+\left(D f\left(v_{\infty}\right)+Q_{\mathrm{s}}(x)+Q_{\mathrm{c}}(x)\right) w_{\star}(x)=0
$$

for $x \in \mathbb{R}^{d}$. If we omit the term $Q_{\mathrm{s}}+Q_{\mathrm{c}}$ in (2.14), the equation (2.14) is called the far-field linearization.

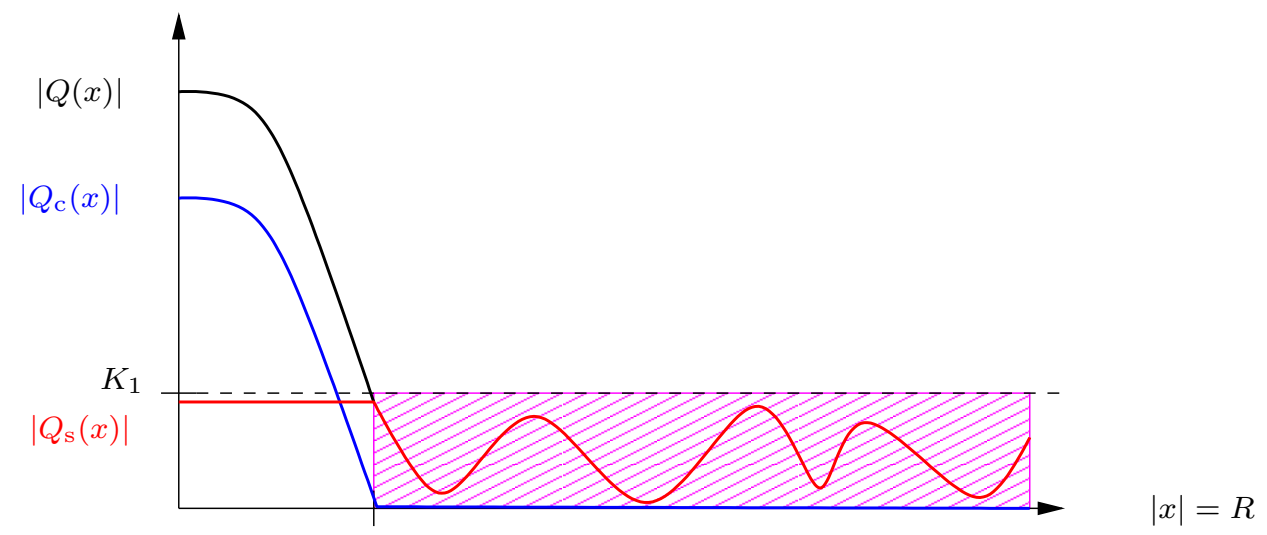

$R_{0}$

Figure 2. Decomposition of $Q$ with data $R_{0}$ and $K_{1}$ from Theorem 2.8

Perturbations of Ornstein-Uhlenbeck operator. In order to show exponential decay for the solution $v_{\star}$ of the nonlinear steady state problem (2.11), it is sufficient to analyze solutions of the linear system (2.14). Abbreviating $B_{\infty}:=-D f\left(v_{\infty}\right)$, we will study the following linear differential operators:

$$
\begin{aligned}
\mathcal{L}_{\mathrm{c}} v & =A \triangle v+\langle S x, \nabla v\rangle-B_{\infty} v+Q_{\mathrm{s}}(x) v+Q_{\mathrm{c}}(x) v, \\
\mathcal{L}_{\mathrm{s}} v & =A \triangle v+\langle S x, \nabla v\rangle-B_{\infty} v+Q_{\mathrm{s}}(x) v, \\
\mathcal{L}_{\infty} v & =A \triangle v+\langle S x, \nabla v\rangle-B_{\infty} v, \\
\mathcal{L}_{0} v & =A \triangle v+\langle S x, \nabla v\rangle .
\end{aligned}
$$

Recall that the drift term $\langle S x, \nabla v(x)\rangle, x \in \mathbb{R}^{d}$, in the Ornstein-Uhlenbeck operator $\mathcal{L}_{0}$ has unbounded coefficients and cannot be considered as a lower order term. Later on, it will be convenient to allow complex coefficients for the operators $\mathcal{L}_{0}$, $\mathcal{L}_{\infty}, \mathcal{L}_{\mathrm{s}}$ and $\mathcal{L}_{\mathrm{c}}$. Therefore, we rewrite the assumptions (A9)-(A11) as follows:

Assumption 2.9. For $B_{\infty} \in \mathbb{K}^{N, N}$ consider the conditions

$\left(\mathrm{A} 9_{B_{\infty}}\right) \quad A, B_{\infty} \in \mathbb{K}^{N, N}$ are simultaneously diagonalizable (over $\mathbb{C}$ ), i.e.

$\exists Y \in \mathbb{C}^{N, N}$ invertible : $Y^{-1} A Y=\Lambda_{A}$ and $Y^{-1} B_{\infty} Y=\Lambda_{B_{\infty}}$, with $\Lambda_{A}=\operatorname{diag}\left(\lambda_{1}^{A}, \ldots, \lambda_{N}^{A}\right), \Lambda_{B_{\infty}}=\operatorname{diag}\left(\lambda_{1}^{B \infty}, \ldots, \lambda_{N}^{B_{\infty}}\right) \in \mathbb{C}^{N, N}$, $\left(\mathrm{A} 10_{B_{\infty}}\right) \operatorname{Re} \sigma\left(B_{\infty}\right)>0$, 
$\left(\mathrm{A} 11_{B_{\infty}}\right)$ There exists $\beta_{\infty}>0$ such that for all $w \in \mathbb{K}^{N}$ with $|w|=1$

$$
\operatorname{Re}\left\langle w, B_{\infty} w\right\rangle \geqslant \beta_{\infty} \text {. }
$$

Similar comments as those following (A9)-(A11) apply. In addition to (2.7), we need the constants

$$
b_{0}:=-s\left(-B_{\infty}\right), \quad \kappa:=\operatorname{cond}(Y) \quad\left(\text { with } Y \text { from }\left(\mathrm{A} 9_{B_{\infty}}\right)\right),
$$

where $\operatorname{cond}(Y):=\left\|Y^{-1}\right\|\|Y\|$ denotes the condition number of $Y$.

2.3. Constant coefficient perturbations of Ornstein-Uhlenbeck operators. In the first step we review and collect results from $[\mathbf{2 6}, \mathbf{2 7}, \mathbf{2 9}, \mathbf{2 8}]$ for the complex-valued Ornstein-Uhlenbeck operator $\mathcal{L}_{0}$ in $L^{p}\left(\mathbb{R}^{d}, \mathbb{C}^{N}\right)$ and its constant coefficient perturbation $\mathcal{L}_{\infty}$.

Assuming (A2), (A6), $\left(\mathrm{A} 9_{B_{\infty}}\right)$ for $\mathbb{K}=\mathbb{C}$ it is shown in [26, Thm.4.2-4.4], [27, Thm.3.1] that the function $H_{\infty}: \mathbb{R}^{d} \times \mathbb{R}^{d} \times(0, \infty) \rightarrow \mathbb{C}^{N, N}$ defined by

$$
H_{\infty}(x, \xi, t)=(4 \pi t A)^{-\frac{d}{2}} \exp \left(-B_{\infty} t-(4 t A)^{-1}\left|e^{t S} x-\xi\right|^{2}\right),
$$

is a heat kernel of the perturbed Ornstein-Uhlenbeck operator $\mathcal{L}_{\infty}$ from (2.15). Under the same assumptions it is proved in [27, Thm.5.3] that the family of mappings

$$
\left[T_{\infty}(t) v\right](x):=\left\{\begin{array}{ll}
\int_{\mathbb{R}^{d}} H_{\infty}(x, \xi, t) v(\xi) d \xi & , t>0 \\
v(x) & , t=0
\end{array} \quad, x \in \mathbb{R}^{d},\right.
$$

generates a strongly continuous semigroup $T_{\infty}(t): L^{p}\left(\mathbb{R}^{d}, \mathbb{C}^{N}\right) \rightarrow L^{p}\left(\mathbb{R}^{d}, \mathbb{C}^{N}\right)$, $t \geqslant 0$, for each $1 \leqslant p<\infty$, which satisfies the following estimate (see $(2.7),(2.16)$ for the constants)

$$
\left\|T_{\infty}(t) v\right\|_{L^{p}} \leqslant \kappa a_{1} e^{-b_{0} t}\|v\|_{L^{p}} \forall t \geqslant 0 .
$$

The semigroup $\left(T_{\infty}(t)\right)_{t \geqslant 0}$ is called the Ornstein-Uhlenbeck semigroup if $B_{\infty}=0$. Otherwise, $\left(T_{\infty}(t)\right)_{t \geqslant 0}$ is a perturbed Ornstein-Uhlenbeck semigroup. The strong continuity of the semigroup justifies to introduce its infinitesimal generator $\mathcal{A}_{p}: L^{p}\left(\mathbb{R}^{d}, \mathbb{C}^{N}\right) \supseteq \mathcal{D}\left(\mathcal{A}_{p}\right) \rightarrow L^{p}\left(\mathbb{R}^{d}, \mathbb{C}^{N}\right)$ via

$$
\mathcal{D}\left(\mathcal{A}_{p}\right):=\left\{v \in L^{p}\left(\mathbb{R}^{d}, \mathbb{C}^{N}\right) \mid \mathcal{A}_{p} v:=\lim _{t \downarrow 0} \frac{T_{\infty}(t) v-v}{t} \text { exists in } L^{p}\left(\mathbb{R}^{d}, \mathbb{C}^{N}\right)\right\} .
$$

An application of abstract semigroup theory yields the unique solvability of the resolvent equation

$$
\left(\lambda I-\mathcal{A}_{p}\right) v=g, \text { for all } g \in L^{p}\left(\mathbb{R}^{d}, \mathbb{C}^{N}\right), \lambda \in \mathbb{C}, \operatorname{Re} \lambda>-b_{0}=s\left(-B_{\infty}\right)
$$

in $L^{p}\left(\mathbb{R}^{d}, \mathbb{C}^{N}\right)$ for $1 \leqslant p<\infty$, [26, Cor.6.7], [27, Cor.5.5]. Combining (2.18) with the representation $\left(\lambda I-\mathcal{A}_{p}\right)^{-1} g=\int_{0}^{\infty} e^{-\lambda s} T_{\infty}(s) g d s$, then the solution $v \in \mathcal{D}\left(\mathcal{A}_{p}\right)$ of (2.20) satisfies

$$
v=\left(\lambda I-\mathcal{A}_{p}\right)^{-1} g=\int_{0}^{\infty} \int_{\mathbb{R}^{d}} e^{-\lambda s} H_{\infty}(\cdot, \xi, s) g(\xi) d \xi d s .
$$

The following a-priori estimate in exponentially weighted $L^{p}$-spaces is based on the integral expression (2.21) and is taken from [27, Thm.5.7]. 
Theorem 2.10 (Existence and uniqueness in weighted $W^{1, p}$-spaces). Let the assumptions (A2), (A6) and $\left(\mathrm{A} 9_{B_{\infty}}\right)$ be satisfied for $\mathbb{K}=\mathbb{C}, 1 \leqslant p<\infty$, and let $0<\varepsilon<1$ and $\lambda \in \mathbb{C}$ with $\operatorname{Re} \lambda>-b_{0}$ be given. Moreover, let $\theta \in C\left(\mathbb{R}^{d}, \mathbb{R}\right)$ be a radially nondecreasing weight function of exponential growth rate $\eta \geqslant 0$ with

$$
0 \leqslant \eta^{2} \leqslant \varepsilon \frac{a_{0}\left(\operatorname{Re} \lambda+b_{0}\right)}{a_{\max }^{2} p^{2}} .
$$

Then, for every $g \in L_{\theta}^{p}\left(\mathbb{R}^{d}, \mathbb{C}^{N}\right)$ there exists a unique solution $v \in \mathcal{D}\left(\mathcal{A}_{p}\right)$ of the resolvent equation

$$
\left(\lambda I-\mathcal{A}_{p}\right) v=g, \quad \text { in } L^{p}\left(\mathbb{R}^{d}, \mathbb{C}^{N}\right) .
$$

The solution satisfies $v \in W_{\theta}^{1, p}\left(\mathbb{R}^{d}, \mathbb{C}^{N}\right)$ and the following estimates

$$
\begin{aligned}
\|v\|_{L_{\theta}^{p}} & \leqslant \frac{C_{0, \varepsilon}}{\operatorname{Re} \lambda+b_{0}}\|g\|_{L_{\theta}^{p}}, \\
\left\|D_{i} v\right\|_{L_{\theta}^{p}} & \leqslant \frac{C_{1, \varepsilon}}{\left(\operatorname{Re} \lambda+b_{0}\right)^{\frac{1}{2}}}\|g\|_{L_{\theta}^{p}}, i=1, \ldots, d,
\end{aligned}
$$

where the $\lambda$-independent constants $C_{0, \varepsilon}, C_{1, \varepsilon}$ are given by

$$
\begin{aligned}
C_{0, \varepsilon} & =C_{\theta} \kappa a_{1}\left(\frac{\Gamma\left(\frac{d+1}{2}\right)}{\Gamma\left(\frac{d}{2}\right)}(\pi \varepsilon)^{\frac{1}{2}}(1-\varepsilon)^{-\frac{d+1}{2}}+{ }_{2} F_{1}\left(\frac{d}{2}, 1 ; \frac{1}{2} ; \varepsilon\right)\right)^{\frac{1}{p}}, \\
C_{1, \varepsilon} & =C_{\theta} \kappa \frac{a_{1}^{\frac{d+1}{d}} \pi^{\frac{1}{2}}}{a_{\text {min }}^{\frac{1}{2}}}\left(\frac{\Gamma\left(\frac{d+1}{2}\right)}{\Gamma\left(\frac{d}{2}\right)}(1-\varepsilon)^{-\frac{d+1}{2}}+\frac{d \varepsilon^{\frac{1}{2}}}{\pi^{\frac{1}{2}}}{ }_{2} F_{1}\left(\frac{d+1}{2}, 1 ; \frac{3}{2} ; \varepsilon\right)\right)^{\frac{1}{p}},
\end{aligned}
$$

with constants $a_{0}, a_{1}, a_{\min }, a_{\max }$ from (2.7), $b_{0}, \kappa$ from (2.16) and $C_{\theta}$ from (W2).

REMARK 2.11. Above we used the hypergeometric function ${ }_{2} F_{1}$, see $[25,15.4]$. Moreover, we modified the original constants from [27, Thm.5.7] by using ${ }_{2} F_{1}(a, b ; b ; z)=$ $(1-z)^{-a}$ from $[25,(15.4 .6)]$ and the Pfaff transformation ${ }_{2} F_{1}(a, b ; c, z)=(1-$ $z)^{-b}{ }_{2} F_{1}\left(c-a, b ; c ; \frac{z}{z-1}\right)$ for $z \in \mathbb{C} \backslash[1, \infty)$. Note that both quantities ${ }_{2} F_{1}\left(\frac{d}{2}, 1 ; \frac{1}{2} ; \varepsilon\right)$ and ${ }_{2} F_{1}\left(\frac{d+1}{2}, 1 ; \frac{3}{2} ; \varepsilon\right)$ behave like $(1-\varepsilon)^{-\frac{d+1}{2}}$ as $\varepsilon \rightarrow 1([25,(15.4 .23)])$, which then also determines the behavior of the constants $C_{0, \varepsilon}$ and $C_{1, \varepsilon}$.

So far, we neither have an explicit representation for the maximal domain $\mathcal{D}\left(\mathcal{A}_{p}\right)$ in terms of Sobolev spaces, nor do we have the relation between the generator $\mathcal{A}_{p}$ and the differential operator $\mathcal{L}_{\infty}$. For this purpose, one has to solve the identification problem, which has been done in [29]. Assuming (A2), (A6) and (A $\left.9_{B_{\infty}}\right)$ for $\mathbb{K}=$ $\mathbb{C}$, it is proved in [29, Thm.3.2] that the Schwartz space $\mathcal{S}\left(\mathbb{R}^{d}, \mathbb{C}^{N}\right)$ is a core of the infinitesimal generator $\left(\mathcal{A}_{p}, \mathcal{D}\left(\mathcal{A}_{p}\right)\right)$ for any $1 \leqslant p<\infty$. Next, one considers $\mathcal{L}_{\infty}: L^{p}\left(\mathbb{R}^{d}, \mathbb{C}^{N}\right) \supseteq \mathcal{D}_{\text {loc }}^{p}\left(\mathcal{L}_{0}\right) \rightarrow L^{p}\left(\mathbb{R}^{d}, \mathbb{C}^{N}\right)$ on its domain

$$
\mathcal{D}_{\text {loc }}^{p}\left(\mathcal{L}_{0}\right):=\left\{v \in W_{\text {loc }}^{2, p}\left(\mathbb{R}^{d}, \mathbb{C}^{N}\right) \cap L^{p}\left(\mathbb{R}^{d}, \mathbb{C}^{N}\right) \mid \mathcal{L}_{0} v \in L^{p}\left(\mathbb{R}^{d}, \mathbb{C}^{N}\right)\right\} .
$$

Under the assumption $(\mathrm{A} 3)$ for $\mathbb{K}=\mathbb{C}$, it is shown in $\left[29\right.$, Lem.4.1] that $\left(\mathcal{L}_{\infty}, \mathcal{D}_{\text {loc }}^{p}\left(\mathcal{L}_{0}\right)\right)$ is a closed operator in $L^{p}\left(\mathbb{R}^{d}, \mathbb{C}^{N}\right)$ for any $1<p<\infty$. Then the $L^{p}$-dissipativity condition (A4) is the key assumption which leads to an energy estimate for the resolvent with respect to the $L^{p}$-norm, see [29, Thm.4.4]. The same argument reappears in Theorem 3.4 below which is an extension of [29, Thm.4.4]. As a direct consequence, the operator $\mathcal{L}_{\infty}$ is dissipative in $L^{p}\left(\mathbb{R}^{d}, \mathbb{C}^{N}\right)$, provided $\beta_{\infty}$ from 
Assumption $\left(\mathrm{A} 11_{B_{\infty}}\right)$ satisfies $\beta_{\infty} \leqslant 0,[29$, Cor.4.6]. Combining these results one can solve the identification problem for $\mathcal{L}_{\infty}$ as follows (see [29, Thm.5.1]).

Theorem 2.12 (Maximal domain, local version). Let the assumptions (A4), (A6) and $\left(\mathrm{A} 9_{B_{\infty}}\right)$ be satisfied for $\mathbb{K}=\mathbb{C}$ and for some $1<p<\infty$, then

$$
\mathcal{D}\left(\mathcal{A}_{p}\right)=\mathcal{D}_{\text {loc }}^{p}\left(\mathcal{L}_{0}\right)
$$

is the maximal domain of $\mathcal{A}_{p}$, where $\mathcal{D}_{\text {loc }}^{p}\left(\mathcal{L}_{0}\right)$ is defined by (2.24). In particular, $\mathcal{A}_{p}$ is the maximal realization of $\mathcal{L}_{\infty}$ in $L^{p}\left(\mathbb{R}^{d}, \mathbb{C}^{N}\right)$, i.e.

$$
\mathcal{A}_{p} v=\mathcal{L}_{\infty} v \quad \forall v \in \mathcal{D}\left(\mathcal{A}_{p}\right) .
$$

Theorem 2.12 shows that, if we restrict $1<p<\infty$ and replace (A2) by the stronger assumption (A4) in Theorem 2.10, we can write $\mathcal{L}_{\infty}$ and $\mathcal{D}_{\text {loc }}^{p}\left(\mathcal{L}_{0}\right)$ instead of $\mathcal{A}_{p}$ and $\mathcal{D}\left(\mathcal{A}_{p}\right)$. This will be crucial in the proof of Theorem 3.2 below. Moreover, we stress again that it is this theorem into which the $L^{p}$-dissipativity condition (A4) enters, see the comments following Assumption 2.2.

2.4. Bootstrapping and regularity. In Section 3 we study the variable coefficient operator

$$
\left[\mathcal{L}_{Q} v\right](x)=A \triangle v(x)+\langle S x, \nabla v(x)\rangle-B_{\infty} v(x)+Q(x) v(x), x \in \mathbb{R}^{d},
$$

and its resolvent equation

$$
\left(\lambda I-\mathcal{L}_{Q}\right) v=g, \quad \text { in } L^{p}\left(\mathbb{R}^{d}, \mathbb{C}^{N}\right)
$$

for $1<p<\infty$ and for different choices of $Q \in L^{\infty}\left(\mathbb{R}^{d}, \mathbb{C}^{N, N}\right)$. In Section 3.1, we first derive an existence and uniqueness result for the resolvent equation (2.26) in $L^{p}\left(\mathbb{R}^{d}, \mathbb{C}^{N}\right)$ for general $Q$ (Theorem 3.1). The proof uses the standard bounded perturbation theorem from abstract semigroup theory as well as Theorem 2.12. In Section 3.2, we then analyze the resolvent equation (2.26) for perturbations $Q=Q_{\mathrm{s}}$ which are small w.r.t. $\|\cdot\|_{L^{\infty}}$. We prove that the unique solution of $(2.26)$ in $L^{p}\left(\mathbb{R}^{d}, \mathbb{C}^{N}\right)$ decays exponentially if the inhomogeneity $g$ does (Theorem 3.2 ). The proof is based on a fixed point argument and uses the results from Theorem 3.1 and Theorem 2.10. In Section 3.3, we study differential operators of the form

$$
\left[\mathcal{L}_{B} v\right](x)=A \triangle v(x)+\langle S x, \nabla v(x)\rangle-B(x) v(x), x \in \mathbb{R}^{d},
$$

where the matrix-valued function $B \in L^{\infty}\left(\mathbb{R}^{d}, \mathbb{C}^{N, N}\right)$ satisfies

$$
\operatorname{Re}\langle w, B(x) w\rangle \geqslant c_{B}|w|^{2} \forall x \in \mathbb{R}^{d} \forall w \in \mathbb{C}^{N}
$$

for some constant $c_{B} \in \mathbb{R}$. We consider two different weight functions $\theta_{1}, \theta_{2}$ satisfying $\theta_{1} \leq C \theta_{2}$, so that $L_{\theta_{2}}^{p}\left(\mathbb{R}^{d}, \mathbb{C}^{N}\right) \subseteq L_{\theta_{1}}^{p}\left(\mathbb{R}^{d}, \mathbb{C}^{N}\right)$, e.g. $\theta_{2}$ may grow while $\theta_{1}$ decays. Then we prove uniqueness of solutions $v$ of $\left(\lambda I-\mathcal{L}_{B}\right) v=g$ in the large space $W_{\text {loc }}^{2, p}\left(\mathbb{R}^{d}, \mathbb{C}^{N}\right) \cap L_{\theta_{1}}^{p}\left(\mathbb{R}^{d}, \mathbb{C}^{N}\right)$ if $g$ is in the small space $L_{\theta_{2}}^{p}\left(\mathbb{R}^{d}, \mathbb{C}^{N}\right)$, and we derive resolvent estimates (Theorem 3.4). The proof generalizes the approach from [26, Thm.5.13] to variable coefficient perturbations and weighted spaces. In Section 3.4 we study the resolvent equation (2.26) for asymptotically small variable coefficient matrices $Q$. We prove that if $|Q(x)|$ falls below a certain threshold at infinity, then every solution $v \in W_{\text {loc }}^{2, p}\left(\mathbb{R}^{d}, \mathbb{C}^{N}\right) \cap L_{\theta_{1}}^{p}\left(\mathbb{R}^{d}, \mathbb{C}^{N}\right)$ of $(2.26)$ in $L_{\text {loc }}^{p}\left(\mathbb{R}^{d}, \mathbb{C}^{N}\right)$ already belongs to the small space $W_{\theta_{2}}^{1, p}\left(\mathbb{R}^{d}, \mathbb{C}^{N}\right)$ if $g \in L_{\theta_{2}}^{p}\left(\mathbb{R}^{d}, \mathbb{C}^{N}\right)$, and $\operatorname{Re} \lambda>-\beta_{\infty}$ (Theorem 3.5). The idea of the proof is to decompose $Q$ into $Q=Q_{\mathrm{s}}+Q_{\mathrm{c}}$, where $Q_{\mathrm{s}} \in L^{\infty}\left(\mathbb{R}^{d}, \mathbb{C}^{N}\right)$ is small w.r.t. $\|\cdot\|_{L^{\infty}}$ and $Q_{\mathrm{c}}$ is compactly supported on 
$\mathbb{R}^{d}$. Then, Theorem 3.2 implies the existence of a solution in the smaller space $W_{\text {loc }}^{2, p}\left(\mathbb{R}^{d}, \mathbb{C}^{N}\right) \cap L_{\theta_{2}}^{p}\left(\mathbb{R}^{d}, \mathbb{C}^{N}\right)$ and Theorem 3.4 yields the uniqueness in the larger space $W_{\text {loc }}^{2, p}\left(\mathbb{R}^{d}, \mathbb{C}^{N}\right) \cap L_{\theta_{1}}^{p}\left(\mathbb{R}^{d}, \mathbb{C}^{N}\right)$. Note that Theorem 3.5 is the core theorem which allows us to analyze exponential decay for both, solutions of the nonlinear problem and solutions of the eigenvalue problem for $\mathcal{L}$.

In Section 4 we prove spatial exponential decay for bounded solutions of the nonlinear problem (2.11) by employing a bootstrapping argument to the linear equation (2.12). Shifting the term with the compactly supported coefficient to the right-hand side, we obtain an inhomogeneity which lies in any weighted $L^{p_{-}}$ space. Applying the previous linear theory then provides exponential decay in space provided the difference $\left|v_{\star}(x)-v_{\infty}\right|$ falls below a certain threshold at infinity. In a second step, assuming additional regularity of the nonlinearity $f$ and the solution $v_{\star}$, we show that the higher order derivatives also decay exponentially in space (Corollary 4.1, Remark 4.2)

$$
v_{\star}-v_{\infty} \in W_{\theta}^{k, p}\left(\mathbb{R}^{d}, \mathbb{R}^{N}\right), \quad \text { if } f \in C^{\max \{2, k-1\}}\left(\mathbb{R}^{N}, \mathbb{R}^{N}\right), v_{\star} \in C^{k+1}\left(\mathbb{R}^{d}, \mathbb{R}^{N}\right) .
$$

This holds for $k \in \mathbb{N}$ and $p \geqslant \frac{d}{2}$ in case $k \geqslant 3$, where $p$ is from (A4). In Section 4.3 we combine this result with Sobolev embeddings to deduce that $v_{\star}-v_{\infty}$ satisfies exponentially weighted pointwise estimates (Corollary 4.3)

$$
\left|D^{\alpha}\left(v_{\star}(x)-v_{\infty}\right)\right| \leqslant C \exp \left(-\mu \sqrt{|x|^{2}+1}\right) \quad \forall x \in \mathbb{R}^{d}, 0 \leqslant \mu \leqslant \varepsilon \frac{\sqrt{a_{0} b_{0}}}{a_{\max } p}
$$

and for every multi-index $\alpha \in \mathbb{N}_{0}^{d}$ with $d<(k-|\alpha|) p$. In Section 4.4 we extend our main result from Theorem 2.8, Corollary 4.1 and Corollary 4.3 to complex-valued systems with $f$ as in (2.5) (Corollary 4.5).

In Section 5 we study spatial exponential decay for solutions of the eigenvalue problem

$$
A \triangle v(x)+\langle S x, \nabla v(x)\rangle+D f\left(v_{\star}(x)\right) v(x)=\lambda v(x), x \in \mathbb{R}^{d}, d \geqslant 2 .
$$

In Section 5.1 we show that every bounded classical solution $v$ of (2.29) decays exponentially in space, in the sense that $v$ belongs to $W_{\theta}^{1, p}\left(\mathbb{R}^{d}, \mathbb{R}^{N}\right)$, provided that its associated eigenvalue $\lambda \in \mathbb{C}$ satisfies $\operatorname{Re} \lambda>-\beta_{\infty}$. In Section 5.2 we apply our result from Section 5.1 to those eigenfunctions which belong to eigenvalues on the imaginary axis. These eigenfunctions are due to equivariance with respect to the action of the Euclidean group and can be calculated explicitly in terms of the profile $v_{\star}$, see Theorem 5.4. In particular, this yields exponential decay of the eigenfunction $v(x)=\left\langle S x, \nabla v_{\star}(x)\right\rangle, x \in \mathbb{R}^{d}$ associated with the eigenvalue $\lambda=0$. As in the nonlinear case we proceed with proving exponential decay of derivatives of eigenfunctions, first in Sobolev spaces and then in a pointwise sense as in (2.28), see Theorem 5.1.

In Section 6 we apply the theory to so called spinning solitons of the cubicquintic complex Ginzburg-Laundau equation (QCGL)

$$
u_{t}=\alpha \Delta u+u\left(\delta+\beta|u|^{2}+\gamma|u|^{4}\right) u,
$$

where $u: \mathbb{R}^{d} \times[0, \infty) \rightarrow \mathbb{C}, d \in\{2,3\}$ and $\alpha, \beta, \gamma, \delta \in \mathbb{C}$ with $\operatorname{Re} \alpha>0$. We derive suitable conditions on the parameters $\alpha, \beta, \gamma, \delta \in \mathbb{C}$ such that Theorem 2.10 and Corollary 4.5 apply. In Section 6.1 we compute the profile and (angular) speed of the spinning solitons. In Section 6.2 we compute spectra and eigenfunctions of 
the associated eigenvalue problem. In Section 6.3 we compare in a final step the theoretical decay rates with numerical rates obtained from numerical data on a large ball. It turns out that the theoretical bounds are surprisingly close to the values found from numerical computations.

\section{Variable coefficient complex Ornstein-Uhlenbeck operators}

In this section we analyze the resolvent equation of the differential operator

$$
\left[\mathcal{L}_{Q} v\right](x)=A \triangle v(x)+\langle S x, \nabla v(x)\rangle-B_{\infty} v(x)+Q(x) v(x), x \in \mathbb{R}^{d},
$$

in $L^{p}\left(\mathbb{R}^{d}, \mathbb{C}^{N}\right)$ for $1<p<\infty$ and for different choices of $Q \in L^{\infty}\left(\mathbb{R}^{d}, \mathbb{C}^{N, N}\right)$.

3.1. Solvability and uniqueness of the resolvent equation. Let us assume $(\mathrm{A} 2),(\mathrm{A} 6)$ and $\left(\mathrm{A} 9_{B_{\infty}}\right)$ for $\mathbb{K}=\mathbb{C}$ and let $\left(\mathcal{A}_{p}, \mathcal{D}\left(\mathcal{A}_{p}\right)\right)$ denote the generator of the strongly continuous semigroup $\left(T_{\infty}(t)\right)_{t \geqslant 0}$ from Section 2.3 on $L^{p}\left(\mathbb{R}^{d}, \mathbb{C}^{N}\right)$ for some $1 \leqslant p<\infty$. Let us introduce the bounded operator

$$
\mathcal{Q}_{p}: L^{p}\left(\mathbb{R}^{d}, \mathbb{C}^{N}\right) \rightarrow L^{p}\left(\mathbb{R}^{d}, \mathbb{C}^{N}\right) \quad \text { with } \quad\left[\mathcal{Q}_{p} v\right](x):=Q(x) v(x), x \in \mathbb{R}^{d} .
$$

Then the bounded perturbation theorem [15, III.1.3] implies that

$$
\mathcal{B}_{p}:=\mathcal{A}_{p}+\mathcal{Q}_{p} \quad \text { with } \quad \mathcal{D}\left(\mathcal{B}_{p}\right):=\mathcal{D}\left(\mathcal{A}_{p}\right)
$$

generates a strongly continuous semigroup $\left(T_{Q}(t)\right)_{t \geqslant 0}$ in $L^{p}\left(\mathbb{R}^{d}, \mathbb{C}^{N}\right)$ satisfying

$$
\left\|T_{Q}(t) v\right\|_{L^{p}} \leqslant \kappa a_{1} e^{\left(-b_{0}+\kappa a_{1}\left\|\mathcal{Q}_{p}\right\|\right) t}\|v\|_{L^{p}} \leqslant \kappa a_{1} e^{\left(-b_{0}+\kappa a_{1}\|Q\|_{\left.L^{\infty}\right) t}\right.}\|v\|_{L^{p}} \forall t \geqslant 0,
$$

where we used (2.19) and the estimate $\left\|\mathcal{Q}_{p}\right\| \leqslant\|Q\|_{L^{\infty}}$ of the $L^{p}$-operator norm. Then an application of [15, II.1.10] yields that the resolvent equation

$$
\left(\lambda I-\mathcal{B}_{p}\right) v=g, \text { in } L^{p}\left(\mathbb{R}^{d}, \mathbb{C}^{N}\right)
$$

for $\lambda \in \mathbb{C}$ with $\operatorname{Re} \lambda>-b_{0}+\kappa a_{1}\|Q\|_{L^{\infty}}$ and $g \in L^{p}\left(\mathbb{R}^{d}, \mathbb{C}^{N}\right)$ admits a unique solution $v \in \mathcal{D}\left(\mathcal{A}_{p}\right)$ which satisfies the resolvent estimate

$$
\|v\|_{L^{p}} \leqslant \frac{\kappa a_{1}}{\operatorname{Re} \lambda-\left(-b_{0}+\kappa a_{1}\|Q\|_{L^{\infty}}\right)}\|g\|_{L^{p}} .
$$

If we restrict $1<p<\infty$ and assume the stronger assumption (A4) (or equivalently (A5)) instead of (A2), an application of Theorem 2.12 yields that $\mathcal{D}\left(\mathcal{B}_{p}\right):=\mathcal{D}\left(\mathcal{A}_{p}\right)=\mathcal{D}_{\text {loc }}^{p}\left(\mathcal{L}_{0}\right)$ and $\mathcal{B}_{p} v:=\mathcal{A}_{p} v+\mathcal{Q}_{p} v=\mathcal{L}_{\infty} v+Q v=\mathcal{L}_{Q}$ for all $v \in \mathcal{D}\left(\mathcal{B}_{p}\right)$. Therefore we can write in the following $\mathcal{L}_{Q}$ and $\mathcal{D}_{\text {loc }}^{p}\left(\mathcal{L}_{0}\right)$ instead of $\mathcal{B}_{p}$ and $\mathcal{D}\left(\mathcal{B}_{p}\right)$. Summarizing, we obtain the following result.

TheOREM 3.1 (Existence and uniqueness in weighted $L^{p}$-spaces). Let the assumptions (A4), (A6), (A $\left.9_{B_{\infty}}\right)$ and $Q \in L^{\infty}\left(\mathbb{R}^{d}, \mathbb{C}^{N, N}\right)$ be satisfied for $\mathbb{K}=\mathbb{C}$ and for some $1<p<\infty$. Moreover, with constants $a_{1}$ from (2.7), $b_{0}, \kappa$ from (2.16), let $\omega:=-b_{0}+\kappa a_{1}\|Q\|_{L^{\infty}}$ and $\lambda \in \mathbb{C}$ with $\operatorname{Re} \lambda>\omega$ be given. Then, for every $g \in L^{p}\left(\mathbb{R}^{d}, \mathbb{C}^{N}\right)$ the resolvent equation

$$
\left(\lambda I-\mathcal{L}_{Q}\right) v=g
$$

admits a unique solution $v \in \mathcal{D}_{\text {loc }}^{p}\left(\mathcal{L}_{0}\right)$. Moreover, the following resolvent estimate holds:

$$
\|v\|_{L^{p}} \leqslant \frac{\kappa a_{1}}{\operatorname{Re} \lambda-\omega}\|g\|_{L^{p}}
$$


3.2. Exponential decay for small perturbations. In the following we use the constants $a_{0}, a_{1}, a_{\max }, a_{\min }$ from $(2.7), b_{0}, \kappa$ from (2.16) and $C_{0, \varepsilon}, C_{1, \varepsilon}$ from Theorem 2.10 without further reference.

TheOrem 3.2 (Existence and uniqueness in weighted $W^{1, p}$-spaces). Let the assumptions (A4), (A6), $\left(\mathrm{A} 9_{B_{\infty}}\right)$ and $\left(\mathrm{A} 10_{B_{\infty}}\right)$ be satisfied for $\mathbb{K}=\mathbb{C}$ and for some $1<p<\infty$. Moreover, let $0<\varepsilon<1, \theta \in C\left(\mathbb{R}^{d}, \mathbb{R}\right)$ be a radially nondecreasing weight function of exponential growth rate

$$
0 \leqslant \eta \leqslant \varepsilon \frac{\sqrt{a_{0} b_{0}}}{a_{\max } p}
$$

and let $Q_{\mathrm{s}} \in L^{\infty}\left(\mathbb{R}^{d}, \mathbb{C}^{N, N}\right)$ satisfy

$$
\left\|Q_{\mathrm{s}}\right\|_{L^{\infty}} \leqslant \frac{\varepsilon b_{0}}{2} \min \left\{\frac{1}{\kappa a_{1}}, \frac{1}{C_{0, \varepsilon}}\right\} .
$$

Further, let $\lambda \in \mathbb{C}$ with $\operatorname{Re} \lambda \geqslant-(1-\varepsilon) b_{0}$ and $g \in L_{\theta}^{p}\left(\mathbb{R}^{d}, \mathbb{C}^{N}\right)$.

Then there exists a unique solution $v \in \mathcal{D}_{\text {loc }}^{p}\left(\mathcal{L}_{0}\right)$ of the resolvent equation

$$
\left(\lambda I-\mathcal{L}_{\mathrm{s}}\right) v=g, \quad \text { in } L^{p}\left(\mathbb{R}^{d}, \mathbb{C}^{N}\right),
$$

which satisfies $v \in W_{\theta}^{1, p}\left(\mathbb{R}^{d}, \mathbb{C}^{N}\right)$. Moreover, the following estimates hold:

$$
\begin{aligned}
\|v\|_{L_{\theta}^{p}} & \leqslant \frac{2 C_{0, \varepsilon}}{\operatorname{Re} \lambda+b_{0}}\|g\|_{L_{\theta}^{p}}, \\
\left\|D_{i} v\right\|_{L_{\theta}^{p}} & \leqslant \frac{2 C_{1, \varepsilon}}{\left(\operatorname{Re} \lambda+b_{0}\right)^{\frac{1}{2}}}\|g\|_{L_{\theta}^{p}}, i=1, \ldots, d .
\end{aligned}
$$

Proof. Our proof proceeds in three steps.

1. Existence and uniqueness in $L^{p}\left(\mathbb{R}^{d}, \mathbb{C}^{N}\right)$ (by Theorem 3.1): Since $\theta$ is nondecreasing we have $g \in L_{\theta}^{p}\left(\mathbb{R}^{d}, \mathbb{C}^{N}\right) \subseteq L^{p}\left(\mathbb{R}^{d}, \mathbb{C}^{N}\right)$, and due to $\operatorname{Re} \lambda \geqslant-(1-\varepsilon) b_{0}$ and (3.4) we have

$$
\operatorname{Re} \lambda \geqslant-(1-\varepsilon) b_{0} \geqslant-b_{0}+\frac{\varepsilon}{2} b_{0}+\kappa a_{1}\left\|Q_{\mathrm{s}}\right\|_{L^{\infty}}>-b_{0}+\kappa a_{1}\left\|Q_{\mathrm{s}}\right\|_{L^{\infty}} .
$$

Thus, an application of Theorem 3.1 implies that there exists a unique solution $v_{1} \in \mathcal{D}_{\text {loc }}^{p}\left(\mathcal{L}_{0}\right)$ of $\left(\lambda I-\mathcal{L}_{\mathrm{s}}\right) v=g$ in $L^{p}\left(\mathbb{R}^{d}, \mathbb{C}^{N}\right)$. In order to verify that $v_{1}$ belongs to $W_{\theta}^{1, p}\left(\mathbb{R}^{d}, \mathbb{C}^{N}\right)$ and satisfies the inequalities (3.5) and (3.6) we must analyze $\left(\lambda I-\mathcal{L}_{\mathrm{s}}\right) v=g$ in $L_{\theta}^{p}\left(\mathbb{R}^{d}, \mathbb{C}^{N}\right)$.

2. Existence in $L_{\theta}^{p}\left(\mathbb{R}^{d}, \mathbb{C}^{N}\right)$ (by a fixed point argument): Our aim is to show that the equation

$$
v=\left(\lambda I-\mathcal{L}_{\infty}\right)^{-1} g+\left(\lambda I-\mathcal{L}_{\infty}\right)^{-1} Q_{\mathrm{s}} v=: F v
$$

in $L_{\theta}^{p}\left(\mathbb{R}^{d}, \mathbb{C}^{N}\right)$ has a unique fixed point $v_{2} \in L_{\theta}^{p}\left(\mathbb{R}^{d}, \mathbb{C}^{N}\right)$ which even belongs to $\mathcal{D}_{\text {loc }}^{p}\left(\mathcal{L}_{0}\right)$ and agrees with $v_{1}$. For this purpose, consider in $L^{p}\left(\mathbb{R}^{d}, \mathbb{C}^{N}\right)$ the equation

$$
\left(\lambda I-\mathcal{L}_{\infty}\right) u=g+Q_{\mathrm{s}} v, \quad \text { given } \quad v \in L_{\theta}^{p}\left(\mathbb{R}^{d}, \mathbb{C}^{N}\right) .
$$

First note, that the assumptions of Theorem 2.12 are satisfied. This allows us to write $\mathcal{L}_{\infty}$ and $\mathcal{D}_{\text {loc }}^{p}\left(\mathcal{L}_{0}\right)$ instead of $\mathcal{A}_{p}$ and $\mathcal{D}\left(\mathcal{A}_{p}\right)$ in Theorem 2.10. Further, $\operatorname{Re} \lambda \geqslant-(1-\varepsilon) b_{0}$ and equation (3.3) imply

$$
0 \leqslant \eta^{2} \leqslant \varepsilon^{2} \frac{a_{0} b_{0}}{a_{\max }^{2} p^{2}} \leqslant \varepsilon \frac{a_{0}\left(\operatorname{Re} \lambda+b_{0}\right)}{a_{\max }^{2} p^{2}} .
$$


Then Theorem 2.10 yields a unique solution $u \in \mathcal{D}_{\text {loc }}^{p}\left(\mathcal{L}_{0}\right)$ of $(3.8)$ which satisfies $u \in L_{\theta}^{p}\left(\mathbb{R}^{d}, \mathbb{C}^{N}\right)$. This shows that $F$ maps $L_{\theta}^{p}\left(\mathbb{R}^{d}, \mathbb{C}^{N}\right)$ into itself and satisfies $F v \in \mathcal{D}_{\text {loc }}^{p}\left(\mathcal{L}_{0}\right)$ for every $v \in L_{\theta}^{p}\left(\mathbb{R}^{d}, \mathbb{C}^{N}\right)$. Applying $\left(\lambda I-\mathcal{L}_{\infty}\right)^{-1}$ to both sides in (3.8) shows $u=F v$ with $F$ defined in (3.7). Moreover, $F v \in \mathcal{D}_{\text {loc }}^{p}\left(\mathcal{L}_{0}\right) \cap$ $L_{\theta}^{p}\left(\mathbb{R}^{d}, \mathbb{C}^{N}\right)$. The linear part of $F$ is a contraction due to $(2.22)$ and $(3.4)$

$$
\left\|\left(\lambda I-\mathcal{L}_{\infty}\right)^{-1} Q_{\mathrm{s}} v\right\|_{L_{\theta}^{p}} \leqslant q\|v\|_{L_{\theta}^{p}} \quad \forall v \in L_{\theta}^{p}\left(\mathbb{R}^{d}, \mathbb{C}^{N}\right)
$$

with Lipschitz constant

$$
0 \leqslant q:=\frac{C_{0, \varepsilon}}{\operatorname{Re} \lambda+b_{0}}\left\|Q_{\mathrm{s}}\right\|_{L^{\infty}} \leqslant \frac{C_{0, \varepsilon}}{\varepsilon b_{0}}\left\|Q_{\mathrm{s}}\right\|_{L^{\infty}} \leqslant \frac{1}{2}<1 .
$$

Consequently, $F$ is a contraction in $L_{\theta}^{p}\left(\mathbb{R}^{d}, \mathbb{C}^{N}\right)$. Thus, $F$ has a unique fixed point $v_{2} \in L_{\theta}^{p}\left(\mathbb{R}^{d}, \mathbb{C}^{N}\right)$ satisfying $v_{2}=F v_{2} \in \mathcal{D}_{\text {loc }}^{p}\left(\mathcal{L}_{0}\right)$. Since $L_{\theta}^{p}\left(\mathbb{R}^{d}, \mathbb{C}^{N}\right) \subseteq$ $L^{p}\left(\mathbb{R}^{d}, \mathbb{C}^{N}\right)$, the equality $F v_{2}=v_{2}$ holds in $L^{p}\left(\mathbb{R}^{d}, \mathbb{C}^{N}\right)$ as well, and applying $\left(\lambda I-\mathcal{L}_{\infty}\right)$ to both sides yields $\left(\lambda I-\mathcal{L}_{\mathrm{s}}\right) v_{2}=g$ in $L^{p}\left(\mathbb{R}^{d}, \mathbb{C}^{N}\right)$. By the unique solvability of this equation we conclude $v:=v_{1}=v_{2} \in L_{\theta}^{p}\left(\mathbb{R}^{d}, \mathbb{C}^{N}\right)$.

3. $L_{\theta^{-}}^{p}$ and $W_{\theta}^{1, p}$-estimates (by contraction mapping principle and bootstrapping): The $L_{\theta}^{p}$-estimate follows from the contraction mapping principle and the estimates $(2.22),(3.9)$

$$
\|v\|_{L_{\theta}^{p}} \leqslant \frac{1}{1-q}\|F 0\| \leqslant \frac{2 C_{0, \varepsilon}}{\operatorname{Re} \lambda+b_{0}}\|g\|_{L_{\theta}^{p}} .
$$

Finally, the $W_{\theta}^{1, p}$-estimate is proved by bootstrapping using the $L_{\theta}^{p}$-estimate (3.5), the smallness condition (3.4) and (2.23) for every $i=1, \ldots, d$

$$
\begin{aligned}
\left\|D_{i} v\right\|_{L_{\theta}^{p}} & \leqslant \frac{C_{1, \varepsilon}}{\left(\operatorname{Re} \lambda+b_{0}\right)^{\frac{1}{2}}}\left(\|g\|_{L_{\theta}^{p}}+\left\|Q_{\mathrm{s}}\right\|_{L^{\infty}}\|v\|_{L_{\theta}^{p}}\right) \\
& \leqslant \frac{C_{1, \varepsilon}}{\left(\operatorname{Re} \lambda+b_{0}\right)^{\frac{1}{2}}}(1+2 q)\|g\|_{L_{\theta}^{p}} \leqslant \frac{2 C_{1, \varepsilon}}{\left(\operatorname{Re} \lambda+b_{0}\right)^{\frac{1}{2}}}\|g\|_{L_{\theta}^{p}} .
\end{aligned}
$$

3.3. Exponentially weighted resolvent estimates for variable coefficient operators. Consider the differential operator

$$
\left[\mathcal{L}_{B} v\right](x):=A \triangle v(x)+\langle S x, \nabla v(x)\rangle-B(x) v(x), x \in \mathbb{R}^{d} .
$$

The following Lemma 3.3 is crucial to derive energy estimates for $\mathcal{L}_{B}$ in exponentially weighted $L^{p}$-spaces, see Theorem 3.4 below. The result is proved in [29, Lem.4.2], [26, Lem.5.12], it is a vector-valued and complex-valued version of [20, Lem.2.1].

Lemma 3.3. Let the assumption (A3) be satisfied for $\mathbb{K}=\mathbb{C}$. Moreover, let $\Omega \subset \mathbb{R}^{d}$ be a bounded domain with a $C^{2}$-boundary or $\Omega=\mathbb{R}^{d}, 1<p<\infty, v \in$ 
$W^{2, p}\left(\Omega, \mathbb{C}^{N}\right) \cap W_{0}^{1, p}\left(\Omega, \mathbb{C}^{N}\right)$ and $\eta \in C_{b}^{1}(\Omega, \mathbb{R})$ be nonnegative, then

$$
\begin{aligned}
-\operatorname{Re} \int_{\Omega} \eta \bar{v}^{T}|v|^{p-2} A \triangle v & \geqslant \operatorname{Re} \int_{\Omega} \eta|v|^{p-2} \sum_{j=1}^{d}{\overline{D_{j}}}^{T} A D_{j} v \quad\{v \neq 0\} \\
& +\operatorname{Re} \int_{\Omega} \bar{v}^{T}|v|^{p-2} \sum_{j=1}^{d} D_{j} \eta A D_{j} v \\
& +(p-2) \operatorname{Re} \int_{\Omega} \eta|v|^{p-4} \sum_{j=1}^{d} \operatorname{Re}\left({\overline{D_{j} v}}^{T} v\right) \bar{v}^{T} A D_{j} v \quad\{v \neq 0\}
\end{aligned}
$$

Some care has to be taken when using this estimate. By a slight abuse of notation, the term $|v|^{q} \quad\{v \neq 0\}$ in the integrands should be read for powers $q<0$ as follows

$$
\left[|v|^{q} \quad\{v \neq 0\}\right](x)= \begin{cases}|v(x)|^{q}, & |v(x)|>0 \\ 0, & v(x)=0 .\end{cases}
$$

The proof of Lemma 3.3 shows by using Lebesgue's dominated convergence and Fatou's lemma that the integrals involving $\{v \neq 0\}$ exist for $1<p<\infty$, which is nontrivial in case $1<p<2$.

In the following theorem we prove resolvent estimates for $\mathcal{L}_{B}$ in exponentially weighted $L^{p}$-spaces. The theorem extends [26, Thm.5.13] to variable coefficient perturbations of $\mathcal{L}_{0}$ and to weighted $L^{p}$-spaces. Later on, in Theorem 3.5 we apply Theorem 3.4 to $B(x)=B_{\infty}-Q_{\mathrm{s}}(x)$, so that $\mathcal{L}_{B}$ agrees with $\mathcal{L}_{s}$ from (2.15).

ThEOREM 3.4 (Resolvent estimates in weighted $L^{p}$-spaces). Let the assumptions (A4) and (A6) be satisfied for $\mathbb{K}=\mathbb{C}$ and for some $1<p<\infty$. Moreover, let $B \in L^{\infty}\left(\mathbb{R}^{d}, \mathbb{C}^{N, N}\right)$ satisfy the strict accretivity condition

$$
\operatorname{Re}\langle w, B(x) w\rangle \geqslant c_{B}|w|^{2} \forall x \in \mathbb{R}^{d} \forall w \in \mathbb{C}^{N} \text {, for some } c_{B} \in \mathbb{R},
$$

let $\lambda \in \mathbb{C}$ with $\operatorname{Re} \lambda+c_{B}>0$ be given, and let $\theta_{1}, \theta_{2} \in C\left(\mathbb{R}^{d}, \mathbb{R}\right)$ be positive weight functions satisfying

$$
\theta_{1}(x)=\exp \left(-\mu_{1} \sqrt{|x|^{2}+1}\right) \quad \text { with } \quad 0 \leqslant\left|\mu_{1}\right| \leqslant \sqrt{\frac{\left(\operatorname{Re} \lambda+c_{B}\right) \gamma_{A}}{d|A|^{2}}},
$$

and

$$
\theta_{1}(x) \leqslant C \theta_{2}(x) \forall x \in \mathbb{R}^{d} \text { for some } C>0 .
$$

Finally, let $g \in L_{\theta_{2}}^{p}\left(\mathbb{R}^{d}, \mathbb{C}^{N}\right)$ and let $v \in W_{\text {loc }}^{2, p}\left(\mathbb{R}^{d}, \mathbb{C}^{N}\right) \cap L_{\theta_{1}}^{p}\left(\mathbb{R}^{d}, \mathbb{C}^{N}\right)$ be a solution of

$$
\left(\lambda I-\mathcal{L}_{B}\right) v=g \quad \text { in } L_{\mathrm{loc}}^{p}\left(\mathbb{R}^{d}, \mathbb{C}^{N}\right) .
$$

Then, $v$ is the unique solution of $(3.13)$ in $W_{\text {loc }}^{2, p}\left(\mathbb{R}^{d}, \mathbb{C}^{N}\right) \cap L_{\theta_{1}}^{p}\left(\mathbb{R}^{d}, \mathbb{C}^{N}\right)$ and satisfies the estimate

$$
\|v\|_{L_{\theta_{1}}^{p}} \leqslant \frac{2 C^{\frac{1}{p}}}{\operatorname{Re} \lambda+c_{B}}\|g\|_{L_{\theta_{2}}^{p}} .
$$

In addition, for $1<p \leqslant 2$ the following gradient estimate holds

$$
\left\|D_{i} v\right\|_{L_{\theta_{1}}^{p}} \leqslant \frac{2 C^{\frac{1}{p}} \gamma_{A}^{-\frac{1}{2}}}{\left(\operatorname{Re} \lambda+c_{B}\right)^{\frac{1}{2}}}\|g\|_{L_{\theta_{2}}^{p}}, \quad i=1, \ldots, d,
$$


with $C$ from (3.12), $\gamma_{A}$ from (A4) and $c_{B}$ from (3.10).

Proof. Consider $v \in W_{\text {loc }}^{2, p}\left(\mathbb{R}^{d}, \mathbb{C}^{N}\right) \cap L_{\theta_{1}}^{p}\left(\mathbb{R}^{d}, \mathbb{C}^{N}\right)$ which satisfies (3.13) for some $g \in L_{\theta_{2}}^{p}\left(\mathbb{R}^{d}, \mathbb{C}^{N}\right)$. For $n \in \mathbb{R}$ with $n>0$ let us define the cut-off functions

$$
\chi_{n}(x)=\chi_{1}\left(\frac{x}{n}\right), \chi_{1} \in C_{\mathrm{c}}^{\infty}\left(\mathbb{R}^{d}, \mathbb{R}\right), \chi_{1}(x)= \begin{cases}1 & ,|x| \leqslant 1 \\ \in[0,1], \text { smooth } & , 1<|x|<2 . \\ 0 & ,|x| \geqslant 2\end{cases}
$$

1. We multiply (3.13) from left by $\chi_{n}^{2} \theta_{1} \bar{v}^{T}|v|^{p-2}, n \in \mathbb{N}$, integrate over $\mathbb{R}^{d}$ and take real parts,

$$
\begin{aligned}
& \operatorname{Re} \int_{\mathbb{R}^{d}} \chi_{n}^{2} \theta_{1}|v|^{p-2} \bar{v}^{T} g \\
= & (\operatorname{Re} \lambda) \int_{\mathbb{R}^{d}} \chi_{n}^{2} \theta_{1}|v|^{p}-\operatorname{Re} \int_{\mathbb{R}^{d}} \chi_{n}^{2} \theta_{1} \bar{v}^{T}|v|^{p-2} A \triangle v \\
& -\operatorname{Re} \int_{\mathbb{R}^{d}} \chi_{n}^{2} \theta_{1} \bar{v}^{T}|v|^{p-2} \sum_{j=1}^{d}(S x)_{j} D_{j} v+\operatorname{Re} \int_{\mathbb{R}^{d}} \chi_{n}^{2} \theta_{1} \bar{v}^{T}|v|^{p-2} B v .
\end{aligned}
$$

2. Let us rewrite the third term on the right-hand side by using the formula

$$
D_{j}\left(|v|^{p}\right)=p|v|^{p-2} \operatorname{Re}\left({\overline{D_{j} v}}^{T} v\right)
$$

and the following identity obtained from (A6) and integration by parts,

$$
\begin{aligned}
0= & \frac{1}{p} \int_{\mathbb{R}^{d}} \chi_{n}^{2} \theta_{1}\left(\sum_{j=1}^{d} S_{j j}\right)|v|^{p}=\frac{1}{p} \sum_{j=1}^{d} \int_{\mathbb{R}^{d}} \chi_{n}^{2} D_{j}\left((S x)_{j}\right) \theta_{1}|v|^{p} \\
= & -\frac{2}{p} \sum_{j=1}^{d} \int_{\mathbb{R}^{d}} \chi_{n}\left(D_{j} \chi_{n}\right)(S x)_{j} \theta_{1}|v|^{p}-\sum_{j=1}^{d} \int_{\mathbb{R}^{d}} \chi_{n}^{2} \theta_{1}(S x)_{j} \operatorname{Re}\left(\bar{D}_{j}{ }^{T} v\right)|v|^{p-2} \\
& -\frac{1}{p} \sum_{j=1}^{d} \int_{\mathbb{R}^{d}} \chi_{n}^{2}(S x)_{j}\left(D_{j} \theta_{1}\right)|v|^{p} \\
= & -\frac{2}{p} \int_{\mathbb{R}^{d}} \chi_{n} \theta_{1}|v|^{p} \sum_{j=1}^{d}\left(D_{j} \chi_{n}\right)(S x)_{j}-\operatorname{Re} \int_{\mathbb{R}^{d}} \chi_{n}^{2} \theta_{1} \bar{v}^{T}|v|^{p-2} \sum_{j=1}^{d}(S x)_{j} D_{j} v \\
& -\frac{1}{p} \int_{\mathbb{R}^{d}} \chi_{n}^{2}|v|^{p} \sum_{j=1}^{d}(S x)_{j}\left(D_{j} \theta_{1}\right) .
\end{aligned}
$$

We insert this into (3.17) and apply Lemma 3.3 to the second term with $\Omega=$ $B_{2 n}(0), \eta=\chi_{n}^{2} \theta_{1}$

$$
\begin{aligned}
& \operatorname{Re} \int_{\mathbb{R}^{d}} \chi_{n}^{2} \theta_{1}|v|^{p-2} \bar{v}^{T} g=(\operatorname{Re} \lambda) \int_{\mathbb{R}^{d}} \chi_{n}^{2} \theta_{1}|v|^{p}-\operatorname{Re} \int_{\mathbb{R}^{d}} \chi_{n}^{2} \theta_{1} \bar{v}^{T}|v|^{p-2} A \triangle v \\
& +\frac{2}{p} \int_{\mathbb{R}^{d}} \chi_{n} \theta_{1}|v|^{p} \sum_{j=1}^{d}\left(D_{j} \chi_{n}\right)(S x)_{j}+\frac{1}{p} \int_{\mathbb{R}^{d}} \chi_{n}^{2}|v|^{p} \sum_{j=1}^{d}\left(D_{j} \theta_{1}\right)(S x)_{j} \\
& +\operatorname{Re} \int_{\mathbb{R}^{d}} \chi_{n}^{2} \theta_{1} \bar{v}^{T}|v|^{p-2} B v
\end{aligned}
$$




$$
\begin{aligned}
& \geqslant(\operatorname{Re} \lambda) \int_{\mathbb{R}^{d}} \chi_{n}^{2} \theta_{1}|v|^{p}+\frac{2}{p} \int_{\mathbb{R}^{d}} \chi_{n} \theta_{1}|v|^{p} \sum_{j=1}^{d}\left(D_{j} \chi_{n}\right)(S x)_{j} \\
& +\frac{1}{p} \int_{\mathbb{R}^{d}} \chi_{n}^{2}|v|^{p} \sum_{j=1}^{d}\left(D_{j} \theta_{1}\right)(S x)_{j}+\operatorname{Re} \int_{\mathbb{R}^{d}} 2 \chi_{n} \theta_{1} \bar{v}^{T}|v|^{p-2} \sum_{j=1}^{d} D_{j} \chi_{n} A D_{j} v \\
& +\operatorname{Re} \int_{\mathbb{R}^{d}} \chi_{n}^{2} \bar{v}^{T}|v|^{p-2} \sum_{j=1}^{d}\left(D_{j} \theta_{1}\right) A D_{j} v \\
& +(p-2) \operatorname{Re} \int_{\mathbb{R}^{d}} \chi_{n}^{2} \theta_{1}|v|^{p-4} \sum_{j=1}^{d} \operatorname{Re}\left({\overline{D_{j} v}}^{T} v\right) \bar{v}^{T} A D_{j} v \quad\{v \neq 0\} \\
& +\operatorname{Re} \int_{\mathbb{R}^{d}} \chi_{n}^{2} \theta_{1}|v|^{p-2} \sum_{j=1}^{d}{\overline{D_{j}}}^{T} A D_{j} v \quad{ }_{\{v \neq 0\}}+\operatorname{Re} \int_{\mathbb{R}^{d}} \chi_{n}^{2} \theta_{1} \bar{v}^{T}|v|^{p-2} B v .
\end{aligned}
$$

3. Subtracting the 2nd, 3rd, 4th and 5th term of the right hand side, yields the upper bound

$$
\begin{aligned}
& (\operatorname{Re} \lambda) \int_{\mathbb{R}^{d}} \chi_{n}^{2} \theta_{1}|v|^{p}+\operatorname{Re} \int_{\mathbb{R}^{d}} \chi_{n}^{2} \theta_{1}|v|^{p-2} \sum_{j=1}^{d}{\overline{D_{j} v}}^{T} A D_{j} v \quad\{v \neq 0\} \\
& +(p-2) \operatorname{Re} \int_{\mathbb{R}^{d}} \chi_{n}^{2} \theta_{1}|v|^{p-4} \sum_{j=1}^{d} \operatorname{Re}\left(\overline{D_{j} v}{ }^{T} v\right) \bar{v}^{T} A D_{j} v \quad\{v \neq 0\} \\
& +\operatorname{Re} \int_{\mathbb{R}^{d}} \chi_{n}^{2} \theta_{1} \bar{v}^{T}|v|^{p-2} B v \\
& \leqslant \operatorname{Re} \int_{\mathbb{R}^{d}} \chi_{n}^{2} \theta_{1}|v|^{p-2} \bar{v}^{T} g-\operatorname{Re} \int_{\mathbb{R}^{d}} 2 \chi_{n} \theta_{1} \bar{v}^{T}|v|^{p-2} \sum_{j=1}^{d} D_{j} \chi_{n} A D_{j} v \\
& -\frac{2}{p} \int_{\mathbb{R}^{d}} \chi_{n} \theta_{1}|v|^{p} \sum_{j=1}^{d}\left(D_{j} \chi_{n}\right)(S x)_{j}-\frac{1}{p} \int_{\mathbb{R}^{d}} \chi_{n}^{2}|v|^{p} \sum_{j=1}^{d}\left(D_{j} \theta_{1}\right)(S x)_{j} \\
& -\operatorname{Re} \int_{\mathbb{R}^{d}} \chi_{n}^{2} \bar{v}^{T}|v|^{p-2} \sum_{j=1}^{d}\left(D_{j} \theta_{1}\right) A D_{j} v=: T_{1}+T_{2}+T_{3}+T_{4}+T_{5} .
\end{aligned}
$$

We estimate the terms successively. Using Re $z \leqslant|z|$ and (3.12), Hölder's inequality yields

$$
\begin{aligned}
T_{1} & =\int_{\mathbb{R}^{d}} \chi_{n}^{2} \theta_{1}|v|^{p-2} \operatorname{Re}\left(\bar{v}^{T} g\right) \leqslant \int_{\mathbb{R}^{d}} \chi_{n}^{2} \theta_{1}|v|^{p-1}|g| \\
& \leqslant\left(\int_{\mathbb{R}^{d}}\left(\chi_{n}^{\frac{2(p-1)}{p}} \theta_{1}^{\frac{p-1}{p}}|v|^{p-1}\right)^{\frac{p}{p-1}}\right)^{\frac{p-1}{p}}\left(\int_{\mathbb{R}^{d}}\left(\chi_{n}^{\frac{2}{p}} \theta_{1}^{\frac{1}{p}}|g|\right)^{p}\right)^{\frac{1}{p}} \\
& \leqslant C^{\frac{1}{p}}\left(\int_{\mathbb{R}^{d}} \chi_{n}^{2} \theta_{1}|v|^{p}\right)^{\frac{p-1}{p}}\left(\int_{\mathbb{R}^{d}} \chi_{n}^{2} \theta_{2}|g|^{p}\right)^{\frac{1}{p}} .
\end{aligned}
$$


For the 2nd term we use Hölder's inequality with $p=q=2$ and Young's inequality with $\delta>0$

$$
\begin{aligned}
T_{2} & \leqslant 2|A| \int_{\mathbb{R}^{d}} \chi_{n} \theta_{1}|v|^{p-1} \sum_{j=1}^{d}\left|D_{j} \chi_{n}\right|\left|D_{j} v\right| \\
& \leqslant \frac{2|A|\left\|\chi_{1}\right\|_{1, \infty}}{n} \sum_{j=1}^{d} \int_{\mathbb{R}^{d}} \chi_{n} \theta_{1}\left|D_{j} v\right||v|^{p-1} \\
& \leqslant \frac{2|A|\left\|\chi_{1}\right\|_{1, \infty}}{n} \sum_{j=1}^{d}\left(\int_{\mathbb{R}^{d}} \chi_{n}^{2} \theta_{1}\left|D_{j} v\right|^{2}|v|^{p-2}\{v \neq 0\}\right)^{\frac{1}{2}}\left(\int_{\mathbb{R}^{d}} \theta_{1}|v|^{p}\right)^{\frac{1}{2}} \\
& \leqslant \frac{2|A|\left\|\chi_{1}\right\|_{1, \infty}}{n}\left[\delta \sum_{j=1}^{d} \int_{\mathbb{R}^{d}} \chi_{n}^{2} \theta_{1}\left|D_{j} v\right|^{2}|v|^{p-2} \quad\{v \neq 0\}+\frac{d}{4 \delta} \int_{\mathbb{R}^{d}} \theta_{1}|v|^{p}\right] .
\end{aligned}
$$

Here we used that for every $x \in \mathbb{R}^{d}$ and $j=1, \ldots, d$

$$
\left|D_{j} \chi_{n}(x)\right|=\left|D_{j}\left(\chi_{1}\left(\frac{x}{n}\right)\right)\right| \leqslant \frac{1}{n} \max _{j=1, \ldots, d} \max _{y \in \mathbb{R}^{d}}\left|D_{j} \chi_{1}(y)\right|=\frac{\left\|\chi_{1}\right\|_{1, \infty}}{n} .
$$

For the 3 rd term we use $\chi_{n}(x)=0$ for $|x| \geqslant 2 n$ and $D_{j} \chi_{n}(x)=0$ for $|x| \leqslant n$ to obtain

$$
\begin{aligned}
T_{3} & \leqslant \frac{2}{p} \sum_{j=1}^{d} \int_{\mathbb{R}^{d}} \chi_{n} \theta_{1}|v|^{p}\left|(S x)_{j}\right|\left|D_{j} \chi_{n}\right| \\
& =\frac{2}{p} \sum_{j=1}^{d} \int_{n \leqslant|x| \leqslant 2 n} \chi_{n} \theta_{1}|v|^{p}\left|(S x)_{j}\right|\left|D_{j} \chi_{n}\right| \leqslant \frac{4 d|S|\left\|\chi_{1}\right\|_{1, \infty}}{p} \int_{n \leqslant|x| \leqslant 2 n} \theta_{1}|v|^{p} .
\end{aligned}
$$

For the last estimate note that $\chi_{n}(x) \leqslant 1$ and

$$
\begin{aligned}
& \left|(S x)_{j}\right|\left|D_{j} \chi_{n}(x)\right|=\frac{1}{n}\left|(S x)_{j}\right|\left|\left(D_{j} \chi_{1}\right)\left(\frac{x}{n}\right)\right| \leqslant \frac{1}{n}|S||x|\left|\left(D_{j} \chi_{1}\right)\left(\frac{x}{n}\right)\right| \\
\leqslant & \frac{|S|}{n}\left(\sup _{n \leqslant|\xi| \leqslant 2 n}|\xi|\right) \max _{j=1, \ldots, d} \max _{y \in \mathbb{R}^{d}}\left|D_{j} \chi_{1}(y)\right|=2|S|\left\|\chi_{1}\right\|_{1, \infty} .
\end{aligned}
$$

The 4 th term vanishes, as follows from (3.11) and (A6),

$$
T_{4}=-\frac{1}{p} \int_{\mathbb{R}^{d}} \chi_{n}^{2} \frac{-\mu_{1}}{\sqrt{|x|^{2}+1}} \theta_{1}|v|^{p} \sum_{j=1}^{d} x_{j}(S x)_{j}=0 .
$$

For the 5th term we use again $\operatorname{Re} z \leqslant|z|$, Hölder's inequality with $p=q=2$ and Young's inequality with some $\rho>0,(3.11)$ and $\left|\mu_{1}\right| \leqslant \mu_{0}$ for some $\mu_{0} \geqslant 0$ that will be specified below

$$
\begin{aligned}
T_{5} & \leqslant \int_{\mathbb{R}^{d}} \chi_{n}^{2}|v|^{p-1} \sum_{j=1}^{d}\left|\frac{-\mu_{1} x_{j}}{\sqrt{|x|^{2}+1}}\right| \theta_{1}|A|\left|D_{j} v\right| \leqslant\left|\mu_{1}\right||A| \sum_{j=1}^{d} \int_{\mathbb{R}^{d}} \chi_{n}^{2} \theta_{1}|v|^{p-1}\left|D_{j} v\right| \\
& \leqslant\left|\mu_{1}\right||A| \sum_{j=1}^{d}\left(\int_{\mathbb{R}^{d}} \chi_{n}^{2} \theta_{1}|v|^{p-2}\left|D_{j} v\right|^{2} \quad\{v \neq 0\}\right)^{\frac{1}{2}}\left(\int_{\mathbb{R}^{d}} \chi_{n}^{2} \theta_{1}|v|^{p}\right)^{\frac{1}{2}} \\
& \leqslant \frac{\mu_{0}|A|}{4 \rho} \sum_{j=1}^{d} \int_{\mathbb{R}^{d}} \chi_{n}^{2} \theta_{1}|v|^{p-2}\left|D_{j} v\right|^{2} \quad\{v \neq 0\}+\mu_{0}|A| \rho d \int_{\mathbb{R}^{d}} \chi_{n}^{2} \theta_{1}|v|^{p} .
\end{aligned}
$$


Summarizing, we arrive at the following estimate

$$
\begin{aligned}
& (\operatorname{Re} \lambda) \int_{\mathbb{R}^{d}} \chi_{n}^{2} \theta_{1}|v|^{p}+\int_{\mathbb{R}^{d}} \chi_{n}^{2} \theta_{1}|v|^{p-2} \operatorname{Re}\langle v, B v\rangle+\int_{\mathbb{R}^{d}} \chi_{n}^{2} \theta_{1}|v|^{p-4}\{v \neq 0\} \\
\times & \sum_{j=1}^{d}\left[|v|^{2} \operatorname{Re}\left\langle D_{j} v, A D_{j} v\right\rangle+(p-2) \operatorname{Re}\left\langle D_{j} v, v\right\rangle \operatorname{Re}\left\langle v, A D_{j} v\right\rangle\right] \\
\leqslant & C^{\frac{1}{p}}\left(\int_{\mathbb{R}^{d}} \chi_{n}^{2} \theta_{1}|v|^{p}\right)^{\frac{p-1}{p}}\left(\int_{\mathbb{R}^{d}} \chi_{n}^{2} \theta_{2}|g|^{p}\right)^{\frac{1}{p}}+\frac{2 d|A|\left\|\chi_{1}\right\|_{1, \infty}}{4 n \delta} \int_{\mathbb{R}^{d}} \theta_{1}|v|^{p} \\
+ & 2\left\|\chi_{1}\right\|_{1, \infty}\left[\frac{2 d|S|}{p} \int_{n \leqslant|x| \leqslant 2 n} \theta_{1}|v|^{p}+\frac{|A| \delta}{n} \sum_{j=1}^{d} \int_{\mathbb{R}^{d}} \chi_{n}^{2} \theta_{1}\left|D_{j} v\right|^{2}|v|^{p-2}\{v \neq 0\}\right] \\
+ & \frac{\mu_{0}|A|}{4 \rho} \sum_{j=1}^{d} \int_{\mathbb{R}^{d}} \chi_{n}^{2} \theta_{1}|v|^{p-2}\left|D_{j} v\right|^{2} \quad\{v \neq 0\}+\mu_{0}|A| \rho d \int_{\mathbb{R}^{d}} \chi_{n}^{2} \theta_{1}|v|^{p} .
\end{aligned}
$$

4. The $L^{p}$-dissipativity assumption (A4) guarantees positivity of the term appearing in brackets $[\cdots]$ and (3.10) provides a lower bound for $\operatorname{Re}\langle v, B v\rangle$. Therefore, putting the last 3 terms from the right-hand to the left-hand side in the last inequality from step 3 , we obtain

$$
\begin{aligned}
& \quad\left(\gamma_{A}-\frac{\mu_{0}|A|}{4 \rho}-\frac{2|A|\left\|\chi_{1}\right\|_{1, \infty} \delta}{n}\right) \sum_{j=1}^{d} \int_{\mathbb{R}^{d}} \chi_{n}^{2} \theta_{1}\left|D_{j} v\right|^{2}|v|^{p-2}\{v \neq 0\} \\
& +\left(\operatorname{Re} \lambda+c_{B}-\mu_{0}|A| \rho d\right) \int_{\mathbb{R}^{d}} \chi_{n}^{2} \theta_{1}|v|^{p} \\
& \leqslant C^{\frac{1}{p}}\left(\int_{\mathbb{R}^{d}} \chi_{n}^{2} \theta_{1}|v|^{p}\right)^{\frac{p-1}{p}}\left(\int_{\mathbb{R}^{d}} \chi_{n}^{2} \theta_{2}|g|^{p}\right)^{\frac{1}{p}}+\frac{2 d|A|\left\|\chi_{1}\right\|_{1, \infty}}{4 n \delta} \int_{\mathbb{R}^{d}} \theta_{1}|v|^{p} \\
& +\frac{4 d|S|\left\|\chi_{1}\right\|_{1, \infty}}{p} \int_{n \leqslant|x| \leqslant 2 n} \theta_{1}|v|^{p} .
\end{aligned}
$$

Now, we choose $\rho=\sqrt{\frac{\operatorname{Re} \lambda+c_{B}}{4 d \gamma_{A}}}, \mu_{0}=\sqrt{\frac{\left(\operatorname{Re} \lambda+c_{B}\right) \gamma_{A}}{d|A|^{2}}}$ so that

$$
\operatorname{Re} \lambda+c_{B}-\mu_{0}|A| \rho d=\frac{\operatorname{Re} \lambda+c_{B}}{2} \text { and } \quad \gamma_{A}-\frac{\mu_{0}|A|}{4 \rho}=\frac{\gamma_{A}}{2} .
$$

Then our estimate reads

$$
\begin{aligned}
& \left(\frac{\gamma_{A}}{2}-\frac{2|A|\left\|\chi_{1}\right\|_{1, \infty} \delta}{n}\right) \sum_{j=1}^{d} \int_{\mathbb{R}^{d}} \chi_{n}^{2} \theta_{1}\left|D_{j} v\right|^{2}|v|^{p-2}\{v \neq 0\} \\
& +\frac{\operatorname{Re} \lambda+c_{B}}{2} \int_{\mathbb{R}^{d}} \chi_{n}^{2} \theta_{1}|v|^{p} \\
\leqslant & C^{\frac{1}{p}}\left(\int_{\mathbb{R}^{d}} \chi_{n}^{2} \theta_{1}|v|^{p}\right)^{\frac{p-1}{p}}\left(\int_{\mathbb{R}^{d}} \chi_{n}^{2} \theta_{2}|g|^{p}\right)^{\frac{1}{p}}+\frac{2 d|A|\left\|\chi_{1}\right\|_{1, \infty}}{4 n \delta} \int_{\mathbb{R}^{d}} \theta_{1}|v|^{p} \\
& +\frac{4 d|S|\left\|\chi_{1}\right\|_{1, \infty}}{p} \int_{n \leqslant|x| \leqslant 2 n} \theta_{1}|v|^{p} .
\end{aligned}
$$

5. Let us choose $\delta>0$ such that $\frac{\gamma_{A}}{2}-2|A|\left\|\chi_{1}\right\|_{1, \infty} \delta>0$. Then we apply Fatou's Lemma to (3.18) and take the limit inferior $n \rightarrow \infty$. First observe that the terms 
$\chi_{n}^{2} \theta_{1}|v|^{p}$ and $\chi_{n}^{2} \theta_{1}\left(\frac{\gamma_{A}}{2}-\frac{2|A|\|\eta\|_{1, \infty} \delta}{n}\right)\left|D_{j} v\right|^{2}|v|^{p-2} \quad\{v \neq 0\}$ on the left-hand side are positive functions in $L^{1}\left(\mathbb{R}^{d}, \mathbb{R}\right)$ and converge pointwise. The convergence of the integrals on the right-hand side of (3.18) is justified by Lebesgue's dominated convergence theorem. We have the pointwise convergence $\chi_{n}^{2} \theta_{1}|v|^{p} \rightarrow \theta_{1}|v|^{p}$, $\chi_{n}^{2} \theta_{2}|g|^{p} \rightarrow \theta_{2}|g|^{p}, \frac{1}{n} \theta_{1}|v|^{p} \rightarrow 0$ and $\theta_{1}|v|^{p} \quad\{n \leqslant|x| \leqslant 2 n\} \rightarrow 0$ for almost every $x \in$ $\mathbb{R}^{d}$ as $n \rightarrow \infty$. They are dominated by $\left.\left.\left|\chi_{n}^{2} \theta_{1}\right| v\right|^{p}\left|\leqslant \theta_{1}\right| v\right|^{p},\left.\left.\left|\chi_{n}^{2} \theta_{2}\right| g\right|^{p}\left|\leqslant \theta_{2}\right| g\right|^{p}$, $\frac{1}{n} \theta_{1}|v|^{p} \leqslant \theta_{1}|v|^{p}, \theta_{1}|v|^{p} \quad\{n \leqslant|x| \leqslant 2 n\} \leqslant \theta_{1}|v|^{p}$, and the bounds belong to $L^{1}\left(\mathbb{R}^{d}, \mathbb{R}\right)$ since

$v \in L_{\theta_{1}}^{p}\left(\mathbb{R}^{d}, \mathbb{C}^{N}\right)$ and $g \in L_{\theta_{2}}^{p}\left(\mathbb{R}^{d}, \mathbb{C}^{N}\right)$. Thus we arrive at

$$
\begin{aligned}
\frac{\operatorname{Re} \lambda+c_{B}}{2}\|v\|_{L_{\theta_{1}}^{p}}^{p} & \leqslant \frac{\operatorname{Re} \lambda+c_{B}}{2} \int_{\mathbb{R}^{d}} \theta_{1}|v|^{p}+\frac{\gamma_{A}}{2} \sum_{j=1}^{d} \int_{\mathbb{R}^{d}} \theta_{1}\left|D_{j} v\right|^{2}|v|^{p-2}\{v \neq 0\} \\
& \leqslant C^{\frac{1}{p}}\left(\int_{\mathbb{R}^{d}} \theta_{1}|v|^{p}\right)^{\frac{p-1}{p}}\left(\int_{\mathbb{R}^{d}} \theta_{2}|g|^{p}\right)^{\frac{1}{p}}=C^{\frac{1}{p}}\|v\|_{L_{\theta_{1}}^{p}}^{p-1}\|g\|_{L_{\theta_{2}}^{p}} .
\end{aligned}
$$

The $L_{\theta_{1}}^{p}$-resolvent estimate (3.14) follows by dividing both sides by $\frac{\operatorname{Re} \lambda+c_{B}}{2}$ and $\|v\|_{L_{\theta_{1}}^{p}}^{p-1}$.

6. The unique solvability of the resolvent equation $\left(\lambda I-\mathcal{L}_{B}\right) v=g$ in $W_{\text {loc }}^{2, p}\left(\mathbb{R}^{d}, \mathbb{C}^{N}\right) \cap L_{\theta_{1}}^{p}\left(\mathbb{R}^{d}, \mathbb{C}^{N}\right)$ clearly follows from the resolvent estimate (3.14). From step 5 we obtain for every $j=1, \ldots, N$

$$
\int_{\mathbb{R}^{d}} \theta_{1}\left|D_{j} v\right|^{2}|v|^{p-2} \quad\{v \neq 0\} \leqslant \frac{2 C^{\frac{1}{p}}}{\gamma_{A}}\|v\|_{L_{\theta_{1}}^{p}}^{p-1}\|g\|_{L_{\theta_{2}}^{p}} .
$$

We take into account that $\left|D_{j} v\right|=\left|D_{j} v\right|_{\{v \neq 0\}}$ a.e. (see e.g. [41, Cor.2.1.8]) and use the $L^{p}$-resolvent estimate (3.14) to deduce from Hölder's inequality for $1<p \leqslant 2$

$$
\begin{aligned}
\left\|D_{j} v\right\|_{L_{\theta_{1}}^{p}}^{p} & =\int_{\mathbb{R}^{d}} \theta_{1}^{\frac{p}{2}}\left|D_{j} v\right|^{p}|v|^{-\frac{p(2-p)}{2}}\{v \neq 0\} \theta_{1}^{\frac{2-p}{2}}|v|^{\frac{p(2-p)}{2}} \\
& \leqslant\left(\int_{\mathbb{R}^{d}} \theta_{1}\left|D_{j} v\right|^{2}|v|^{p-2}\{v \neq 0\}\right)^{\frac{p}{2}}\left(\int_{\mathbb{R}^{d}} \theta_{1}|v|^{p}\right)^{\frac{2-p}{2}} \\
& \leqslant\left(\frac{4 C^{\frac{2}{p}}}{\left(\operatorname{Re} \lambda+c_{B}\right) \gamma_{A}}\right)^{\frac{p}{2}}\|g\|_{L_{\theta_{2}}^{p}}^{p}
\end{aligned}
$$

3.4. Exponential decay for asymptotically small perturbations. In this section we combine the results of Theorems 3.2 and 3.4 to obtain exponential apriori estimates of solutions to variable coefficient equations when the coefficients become small at infinity.

ThEOREM 3.5 (A-priori estimates in weighted $L^{p}$-spaces). Let the assumptions (A4), (A6), $\left(\mathrm{A} 9_{B_{\infty}}\right)$ and $\left(\mathrm{A} 11_{B_{\infty}}\right)$ be satisfied for $\mathbb{K}=\mathbb{C}$ and some $1<p<\infty$. Consider the radial weight functions

$$
\theta_{j}(x)=\exp \left(\mu_{j} \sqrt{|x|^{2}+1}\right), x \in \mathbb{R}^{d}, j=1,2,
$$


with $\mu_{1}, \mu_{2} \in \mathbb{R}$,

$$
-\sqrt{\varepsilon \frac{\gamma_{A} \beta_{\infty}}{2 d|A|^{2}}} \leqslant \mu_{1} \leqslant 0 \leqslant \mu_{2} \leqslant \varepsilon \frac{\sqrt{a_{0} b_{0}}}{a_{\max } p}
$$

for some $0<\varepsilon<1$. Moreover, let $Q \in L^{\infty}\left(\mathbb{R}^{d}, \mathbb{C}^{N, N}\right)$ with

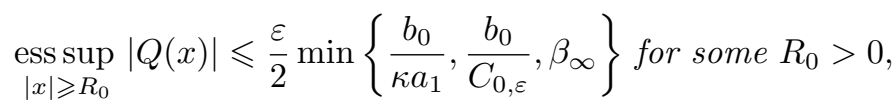

let $g \in L_{\theta_{2}}^{p}\left(\mathbb{R}^{d}, \mathbb{C}^{N}\right)$, and let $\lambda \in \mathbb{C}$ with $\operatorname{Re} \lambda \geqslant-(1-\varepsilon) \beta_{\infty}$ be given.

Then every solution $v \in W_{\mathrm{loc}}^{2, p}\left(\mathbb{R}^{d}, \mathbb{C}^{N}\right) \cap L_{\theta_{1}}^{p}\left(\mathbb{R}^{d}, \mathbb{C}^{N}\right)$ of the resolvent equation

$$
\left(\lambda I-\mathcal{L}_{Q}\right) v=g, \quad \text { in } L_{\text {loc }}^{p}\left(\mathbb{R}^{d}, \mathbb{C}^{N}\right)
$$

satisfies $v \in W_{\theta_{2}}^{1, p}\left(\mathbb{R}^{d}, \mathbb{C}^{N}\right)$. Moreover, the following estimates hold:

$$
\begin{aligned}
\|v\|_{L_{\theta_{2}}^{p}} & \leqslant \frac{2 C_{0, \varepsilon}}{\operatorname{Re} \lambda+b_{0}}\left(C\|v\|_{L_{\theta_{1}}^{p}}+\|g\|_{L_{\theta_{2}}^{p}}\right), \\
\left\|D_{i} v\right\|_{L_{\theta_{2}}^{p}} & \leqslant \frac{2 C_{1, \varepsilon}}{\left(\operatorname{Re} \lambda+b_{0}\right)^{\frac{1}{2}}}\left(C\|v\|_{L_{\theta_{1}}^{p}}+\|g\|_{L_{\theta_{2}}^{p}}\right), \quad i=1, \ldots, d,
\end{aligned}
$$

with constants $a_{0}, a_{1}, a_{\max }$ from (2.7), $\gamma_{A}$ from (A4), $b_{0}, \kappa$ from $(2.16), \beta_{\infty}$ from $\left(\mathrm{A} 11_{B_{\infty}}\right), C:=\exp \left(\left(\mu_{2}-\mu_{1}\right)\left(4 R_{0}^{2}+1\right)^{\frac{1}{2}}\right)\|Q\|_{L^{\infty}}$, and $C_{0, \varepsilon}, C_{1, \varepsilon}$ from Theorem 2.10 (with $C_{\theta}=1$ ).

REMARK 3.6. Note that the exponential decay rate $\mu_{2}$ in (3.20) depends on the spectral data $a_{0}, b_{0}, a_{\max }$, while the growth rate $\theta_{1}$ allowing uniqueness, depends on the norm and accretivity data $\gamma_{A}, \beta_{\infty},|A|$.

Proof. The proof is structured as follows: First we decompose of $Q$ into the sum of $Q_{\mathrm{s}}$ and $Q_{\mathrm{c}}$, where $Q_{\mathrm{s}}$ is small according to (3.21) and $Q_{\mathrm{c}}$ is compactly supported on $\mathbb{R}^{d}$ (step 1$)$. We then consider $\left(\lambda I-\mathcal{L}_{\mathrm{s}}\right) u=Q_{\mathrm{c}} v+g$ and prove existence of a solution in the smaller space $W_{\text {loc }}^{2, p}\left(\mathbb{R}^{d}, \mathbb{C}^{N}\right) \cap L_{\theta_{2}}^{p}\left(\mathbb{R}^{d}, \mathbb{C}^{N}\right)$ by an application of Theorem 3.2 (step 2) and uniqueness in the larger space $W_{\text {loc }}^{2, p}\left(\mathbb{R}^{d}, \mathbb{C}^{N}\right) \cap L_{\theta_{1}}^{p}\left(\mathbb{R}^{d}, \mathbb{C}^{N}\right)$ by an application of Theorem 3.4 (step 3).

1. Decomposition of $Q$ : With the cut-off function $\chi_{R_{0}}$ from (3.16) and $R_{0}$ from (3.21) let us write

$$
Q(x)=Q_{\mathrm{s}}(x)+Q_{\mathrm{c}}(x), \quad Q_{\mathrm{s}}(x):=\left(1-\chi_{R_{0}}(x)\right) Q(x), \quad Q_{\mathrm{c}}(x):=\chi_{R_{0}}(x) Q(x) .
$$

Then $Q_{\mathrm{c}}$ is compactly supported and $Q_{\mathrm{s}}$ satisfies due to (3.21)

$$
\left\|Q_{\mathrm{s}}\right\|_{L^{\infty}} \leqslant\left\|1-\chi_{R_{0}}\right\|_{\infty}\|Q\|_{L^{\infty}\left(\mathbb{R}^{d} \backslash B_{R_{0}}, \mathbb{C}^{N, N}\right)} \leqslant \frac{\varepsilon}{2} \min \left\{\frac{b_{0}}{\kappa a_{1}}, \frac{b_{0}}{C_{0, \varepsilon}}, \beta_{\infty}\right\} .
$$

Let $v \in W_{\text {loc }}^{2, p}\left(\mathbb{R}^{d}, \mathbb{C}^{N}\right) \cap L_{\theta_{1}}^{p}\left(\mathbb{R}^{d}, \mathbb{C}^{N}\right)$ be a solution of $\left(\lambda I-\mathcal{L}_{Q}\right) v=g$ with $\operatorname{Re} \lambda \geqslant$ $-(1-\varepsilon) \beta_{\infty}$ and $g \in L_{\theta_{2}}^{p}\left(\mathbb{R}^{d}, \mathbb{C}^{N}\right)$. Then $v$ satisfies the equation $\left(\lambda I-\mathcal{L}_{\mathrm{s}}\right) v=$ $Q_{\mathrm{c}} v+g$ in $L_{\text {loc }}^{p}\left(\mathbb{R}^{d}, \mathbb{C}^{N}\right)$. Therefore, we consider the problem

$$
\left(\lambda I-\mathcal{L}_{\mathrm{s}}\right) u=Q_{\mathrm{c}} v+g \text {, in } L_{\theta_{1}}^{p}\left(\mathbb{R}^{d}, \mathbb{C}^{N}\right) \text { and in } L_{\theta_{2}}^{p}\left(\mathbb{R}^{d}, \mathbb{C}^{N}\right) .
$$


2. Existence in $W_{\text {loc }}^{2, p}\left(\mathbb{R}^{d}, \mathbb{C}^{N}\right) \cap L_{\theta_{2}}^{p}\left(\mathbb{R}^{d}, \mathbb{C}^{N}\right)$ : Let us apply Theorem 3.2 to (3.24) with $\theta=\theta_{2}, \eta=\left|\mu_{2}\right|$ and $Q_{\mathrm{c}} v+g$ instead of $g$. Note that $Q_{\mathrm{c}} v+g$ belongs to $L_{\theta_{2}}^{p}\left(\mathbb{R}^{d}, \mathbb{C}^{N}\right)$ since

$$
\begin{aligned}
& \left\|Q_{\mathrm{c}} v+g\right\|_{L_{\theta_{2}}^{p}} \leqslant\left\|\theta_{2} Q_{\mathrm{c}} v\right\|_{L^{p}}+\|g\|_{L_{\theta_{2}}^{p}} \\
\leqslant & \left\|\theta_{2} \theta_{1}^{-1}\right\|_{L^{\infty}\left(B_{2 R_{0}}, \mathbb{R}\right)}\|Q\|_{L^{\infty}}\|v\|_{L_{\theta_{1}}^{p}}+\|g\|_{L_{\theta_{2}}^{p}}=C\|v\|_{L_{\theta_{1}}^{p}}+\|g\|_{L_{\theta_{2}}^{p}},
\end{aligned}
$$

with $C:=\exp \left(\left(\mu_{2}-\mu_{1}\right)\left(4 R_{0}^{2}+1\right)^{\frac{1}{2}}\right)\|Q\|_{L^{\infty}}$. Further, $\left(\mathrm{A} 10_{B_{\infty}}\right)$ follows from $\left(\mathrm{A} 11_{B_{\infty}}\right), \beta_{\infty} \leqslant b_{0}$ and $\varepsilon<1$ imply $\operatorname{Re} \lambda \geqslant-(1-\varepsilon) b_{0}$ and $\theta_{2}$ is radially nondecreasing since $\mu_{2} \geqslant 0$. Theorem 3.2 yields that there exists a (unique) $u \in \mathcal{D}_{\text {loc }}^{p}\left(\mathcal{L}_{0}\right) \subset W_{\text {loc }}^{2, p}\left(\mathbb{R}^{d}, \mathbb{C}^{N}\right) \cap L^{p}\left(\mathbb{R}^{d}, \mathbb{C}^{N}\right)$ which solves $(3.24)$ in $L^{p}\left(\mathbb{R}^{d}, \mathbb{C}^{N}\right)$. Moreover, Theorem 3.2 assures $u \in W_{\theta_{2}}^{1, p}\left(\mathbb{R}^{d}, \mathbb{C}^{N}\right)$ as well as the estimates (3.5) and (3.6).

3. Uniqueness in $W_{\text {loc }}^{2, p}\left(\mathbb{R}^{d}, \mathbb{C}^{N}\right) \cap L_{\theta_{1}}^{p}\left(\mathbb{R}^{d}, \mathbb{C}^{N}\right)$ : Consider $(3.24)$ in $L_{\theta_{1}}^{p}\left(\mathbb{R}^{d}, \mathbb{C}^{N}\right)$. We apply Theorem 3.4 with $B(x)=B_{\infty}-Q_{\mathrm{s}}(x)$ and $Q_{\mathrm{c}} v+g$ instead of $g$. First, $B \in L^{\infty}\left(\mathbb{R}^{d}, \mathbb{C}^{N, N}\right)$ follows from $B_{\infty} \in \mathbb{C}^{N, N}$ and $Q_{\mathrm{s}} \in L^{\infty}\left(\mathbb{R}^{d}, \mathbb{C}^{N, N}\right)$. Then, strict accretivity $(3.10)$ with $c_{B}=\left(1-\frac{\varepsilon}{2}\right) \beta_{\infty}$ is a consequence of $\left(\mathrm{A} 11_{B_{\infty}}\right)$ and $\left\|Q_{\mathrm{s}}\right\|_{L^{\infty}} \leqslant \varepsilon \frac{\beta_{\infty}}{2}$,

$\operatorname{Re}\langle w, B(x) w\rangle \geqslant\left(\beta_{\infty}-\left\|Q_{\mathrm{s}}\right\|_{L^{\infty}}\right)|w|^{2} \geqslant\left(1-\frac{\varepsilon}{2}\right) \beta_{\infty}|w|^{2} \quad \forall w \in \mathbb{C}^{N} \forall x \in \mathbb{R}^{d}$.

Moreover, we have $\operatorname{Re} \lambda \geqslant-(1-\varepsilon) \beta_{\infty} \geqslant-\left(1-\frac{\varepsilon}{2}\right) \beta_{\infty}=-c_{B}$. The growth bound in (3.11) is implied by (3.20) and $\operatorname{Re} \lambda+c_{B} \geqslant \frac{\varepsilon}{2} \beta_{\infty}$,

$$
0 \leqslant\left|\mu_{1}\right| \leqslant \sqrt{\varepsilon \frac{\beta_{\infty} \gamma_{A}}{2 d|A|^{2}}} \leqslant \sqrt{\frac{\left(\operatorname{Re} \lambda+c_{B}\right) \gamma_{A}}{d|A|^{2}}} .
$$

Finally, inequality (3.12) is obvious with $C=1$, since $\mu_{1} \leqslant 0 \leqslant \mu_{2}$. Let $u \in$ $W_{\text {loc }}^{2, p}\left(\mathbb{R}^{d}, \mathbb{C}^{N}\right) \cap L_{\theta_{2}}^{p}\left(\mathbb{R}^{d}, \mathbb{C}^{N}\right) \subseteq W_{\text {loc }}^{2, p}\left(\mathbb{R}^{d}, \mathbb{C}^{N}\right) \cap L_{\theta_{1}}^{p}\left(\mathbb{R}^{d}, \mathbb{C}^{N}\right)$ denote the solution from step 2 of the equation $\left(\lambda I-\mathcal{L}_{\mathrm{s}}\right) u=Q_{\mathrm{c}} v+g$ in $L_{\text {loc }}^{p}\left(\mathbb{R}^{d}, \mathbb{C}^{N}\right)$. Since the given $v \in W_{\text {loc }}^{2, p}\left(\mathbb{R}^{d}, \mathbb{C}^{N}\right) \cap L_{\theta_{1}}^{p}\left(\mathbb{R}^{d}, \mathbb{C}^{N}\right)$ solves the same equation, the difference $w=u-v$ solves the homogeneous equation $\left(\lambda I-\mathcal{L}_{\mathrm{c}}\right) w=0$ in $L_{\theta_{1}}^{p}\left(\mathbb{R}^{d}, \mathbb{C}^{N}\right)$, and Theorem 3.4 implies $\|w\|_{L_{\theta_{1}}^{p}}=0$. Therefore, we obtain $v=u \in W_{\theta_{2}}^{1, p}\left(\mathbb{R}^{d}, \mathbb{C}^{N}\right)$.

4. $L_{\theta_{2}}^{p}$ - and $W_{\theta_{2}}^{1, p}$-estimates: The $L_{\theta_{2}}^{p}$-estimate follows from (3.5) and (3.25)

$\|v\|_{L_{\theta_{2}}^{p}}=\|u\|_{L_{\theta_{2}}^{p}} \leqslant \frac{2 C_{0, \varepsilon}}{\operatorname{Re} \lambda+b_{0}}\left\|Q_{\mathrm{c}} v+g\right\|_{L_{\theta_{2}}^{p}} \leqslant \frac{2 C_{0, \varepsilon}}{\operatorname{Re} \lambda+b_{0}}\left(C\|v\|_{L_{\theta_{1}}^{p}}+\|g\|_{L_{\theta_{2}}^{p}}\right)$.

Analogously, the $W_{\theta_{2}}^{1, p}$-estimate follows from (3.6) and (3.25).

\section{Exponential decay of rotating nonlinear waves}

\subsection{Proof of main result.}

Proof (of Theorem 2.8). The proof is structured as follows: First we decompose the nonlinearity $f\left(v_{\star}(x)\right)$ and derive an equation $\mathcal{L}_{Q} w_{\star}=0$ solved by $w_{\star}=v_{\star}-v_{\infty}$ (step 1). Then we apply Theorem 3.5 (step 2) and check its assumptions (steps 3,4). 
1. Let $v_{\star}$ be a classical solution of (2.8) satisfying (2.9). Note that this implies $v_{\star} \in C_{\mathrm{b}}\left(\mathbb{R}^{d}, \mathbb{R}^{N}\right)$. Using (A7) and (A8) we obtain from the mean value theorem

$$
\begin{aligned}
f\left(v_{\star}(x)\right)= & f\left(v_{\infty}\right)+D f\left(v_{\infty}\right)\left(v_{\star}(x)-v_{\infty}\right) \\
& +\int_{0}^{1}\left(D f\left(v_{\infty}+t\left(v_{\star}(x)-v_{\infty}\right)\right)-D f\left(v_{\infty}\right)\right) d t\left(v_{\star}(x)-v_{\infty}\right) \\
= & -B_{\infty}\left(v_{\star}(x)-v_{\infty}\right)+Q(x)\left(v_{\star}(x)-v_{\infty}\right), x \in \mathbb{R}^{d}
\end{aligned}
$$

with

$$
B_{\infty}:=-D f\left(v_{\infty}\right), Q(x):=\int_{0}^{1}\left(D f\left(v_{\infty}+t\left(v_{\star}(x)-v_{\infty}\right)\right)-D f\left(v_{\infty}\right)\right) d t .
$$

For $w_{\star}:=v_{\star}-v_{\infty}$, we have $w_{\star} \in C^{2}\left(\mathbb{R}^{d}, \mathbb{R}^{N}\right) \cap C_{\mathrm{b}}\left(\mathbb{R}^{d}, \mathbb{R}^{N}\right)$ and

$$
\begin{aligned}
0= & A \triangle v_{\star}(x)+\left\langle S x, \nabla v_{\star}(x)\right\rangle+f\left(v_{\star}(x)\right) \\
= & A \triangle\left(v_{\star}(x)-v_{\infty}\right)+\left\langle S x, \nabla\left(v_{\star}(x)-v_{\infty}\right)\right\rangle \\
& -B_{\infty}\left(v_{\star}(x)-v_{\infty}\right)+Q(x)\left(v_{\star}(x)-v_{\infty}\right) \\
= & A \triangle w_{\star}(x)+\left\langle S x, \nabla w_{\star}(x)\right\rangle-B_{\infty} w_{\star}(x)+Q(x) w_{\star}(x) \\
= & {\left[\mathcal{L}_{Q} w_{\star}\right](x), x \in \mathbb{R}^{d} . }
\end{aligned}
$$

2. Let us apply Theorem 3.5 with $B_{\infty}, Q$ from (4.1), $\theta_{2}=\theta, \mu_{2}=\mu, \mu_{1}<0, \lambda=0$ and $g=0$. For this purpose, we have to check the assumptions: Assumptions (A4) and (A6) are directly satisfied, $\left(\mathrm{A} 9_{B_{\infty}}\right)$ follows from (A9), and $\left(\mathrm{A} 11_{B_{\infty}}\right)$ from (A11), using the relation $B_{\infty}=-D f\left(v_{\infty}\right)$. In the following let $0<\varepsilon<1$ be fixed and let $\theta_{1}, \theta_{2} \in C\left(\mathbb{R}^{d}, \mathbb{R}\right)$ be given by (3.19) satisfying $\mu_{1}<0$ and (3.20). First, note that $w_{\star} \in W_{\text {loc }}^{2, p}\left(\mathbb{R}^{d}, \mathbb{C}^{N}\right) \cap L_{\theta_{1}}^{p}\left(\mathbb{R}^{d}, \mathbb{C}^{N}\right)$ follows from $w_{\star} \in$ $C^{2}\left(\mathbb{R}^{d}, \mathbb{R}^{N}\right) \cap C_{\mathrm{b}}\left(\mathbb{R}^{d}, \mathbb{R}^{N}\right)$ and $C_{\mathrm{b}}\left(\mathbb{R}^{d}, \mathbb{R}^{N}\right) \subset L_{\theta_{1}}^{p}\left(\mathbb{R}^{d}, \mathbb{C}^{N}\right)$ due to $\mu_{1}<0$. It remains to verify that $Q \in L^{\infty}\left(\mathbb{R}^{d}, \mathbb{C}^{N, N}\right)$ (step 3) and that (3.21) is satisfied.

3. Since $w_{\star} \in C_{\mathrm{b}}\left(\mathbb{R}^{d}, \mathbb{R}^{N}\right)$ we obtain

$$
\left|v_{\infty}+t w_{\star}(x)\right| \leqslant\left|v_{\infty}\right|+t\left|w_{\star}(x)\right| \leqslant\left|v_{\infty}\right|+\left\|w_{\star}\right\|_{\infty}=: R_{1}
$$

for every $x \in \mathbb{R}^{d}$ and $0 \leqslant t \leqslant 1$. Due to (A7), we have $f \in C^{1}\left(\mathbb{R}^{N}, \mathbb{R}^{N}\right)$ which implies

$$
\begin{aligned}
|Q(x)| & \leqslant \int_{0}^{1}\left|D f\left(v_{\infty}+t w_{\star}(x)\right)\right|+\left|D f\left(v_{\infty}\right)\right| d t \\
& \leqslant \sup _{z \in B_{R_{1}}(0)}|D f(z)|+\left|D f\left(v_{\infty}\right)\right|<\infty
\end{aligned}
$$

for all $x \in \mathbb{R}^{d}$. We deduce $Q \in C_{\mathrm{b}}\left(\mathbb{R}^{d}, \mathbb{R}^{N, N}\right) \subset L^{\infty}\left(\mathbb{R}^{d}, \mathbb{C}^{N, N}\right)$ by taking the suprema over $x \in \mathbb{R}^{d}$.

4. We finally verify (3.21): Let us choose $K_{1}=K_{1}\left(A, f, v_{\infty}, d, p, \varepsilon\right)>0$ such that

$$
K_{1}\left(\sup _{z \in B_{K_{1}}\left(v_{\infty}\right)}\left|D^{2} f(z)\right|\right) \leqslant \varepsilon \min \left\{\frac{b_{0}}{\kappa a_{1}}, \frac{b_{0}}{C_{0, \varepsilon}}, \beta_{\infty}\right\}=: K(\varepsilon)
$$

is satisfied, with the constants $C_{0, \varepsilon}=C_{0, \varepsilon}(A, d, p, \varepsilon, \kappa)$ from Theorem 2.10, $b_{0}:=$ $-s\left(D f\left(v_{\infty}\right)\right)$ and $a_{1}$ from (2.7), $\beta_{\infty}$ from (A11), and

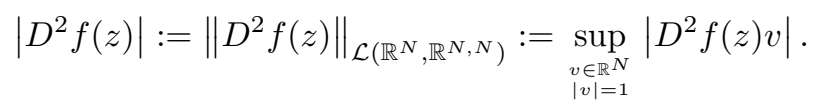


Since $f \in C^{2}\left(\mathbb{R}^{N}, \mathbb{R}^{N}\right)$ by (A7), inequalities (2.9) and (4.2) lead to

$$
\begin{aligned}
|Q(x)| & =\left|\int_{0}^{1} D f\left(v_{\infty}+t w_{\star}(x)\right)-D f\left(v_{\infty}\right) d t\right| \\
& =\left|\int_{0}^{1} \int_{0}^{1} D^{2} f\left(v_{\infty}+s t w_{\star}(x)\right)\left[t w_{\star}(x)\right] d s d t\right| \\
& \leqslant \int_{0}^{1} \int_{0}^{1} \sup _{|x| \geqslant R_{0}}\left|D^{2} f\left(v_{\infty}+s t\left(v_{\star}(x)-v_{\infty}\right)\right)\right| d s \cdot t \sup _{|x| \geqslant R_{0}}\left|v_{\star}(x)-v_{\infty}\right| d t \\
& \leqslant \frac{K_{1}}{2}\left(\sup _{z \in B_{K_{1}}\left(v_{\infty}\right)}\left|D^{2} f(z)\right|\right) \leqslant \frac{\varepsilon}{2} \min \left\{\frac{b_{0}}{\kappa a_{1}}, \frac{b_{0}}{C_{0, \varepsilon}}, \beta_{\infty}\right\}
\end{aligned}
$$

for every $|x| \geqslant R_{0}$. Taking the supremum over $|x| \geqslant R_{0}$ yields condition (3.21). This justifies to apply Theorem 3.5 which shows $w_{\star}=v_{\star}-v_{\infty} \in W_{\theta}^{1, p}\left(\mathbb{R}^{d}, \mathbb{R}^{N}\right)$.

4.2. Exponential decay of higher order derivatives. For estimating higher order derivatives, recall the Sobolev embedding for $0 \leqslant l \leqslant k$,

$$
W^{k, p}\left(\mathbb{R}^{d}, \mathbb{R}^{N}\right) \subseteq W^{l, q}\left(\mathbb{R}^{d}, \mathbb{R}^{N}\right), \quad \text { if } 1<p<q \leqslant \infty, \quad \frac{d}{p}-k \leqslant \frac{d}{q}-l,
$$

where at least one of the inequalities ' $\leqslant$ ' is strict. Moreover, the embedding is continuous, i.e.

$$
\exists C_{p, q, k, l}>0:\|u\|_{W^{l, q}\left(\mathbb{R}^{d}\right)} \leqslant C_{p, q, k, l}\|u\|_{W^{k, p}\left(\mathbb{R}^{d}\right)} \forall u \in W^{k, p}\left(\mathbb{R}^{d}\right) .
$$

For the Sobolev embedding we refer to [2, Thm.5.4], [24, Ch.6], [4, Ch.8] as general reference, and to [35, Thm.3, Exer.24] for the compact version used in (4.3). For the corresponding weighted spaces it is important to note that

$$
u \in W_{\theta}^{k, p}\left(\mathbb{R}^{d}, \mathbb{R}^{N}\right) \Longrightarrow \theta D^{\alpha} u \in W^{k-|\alpha|, p}\left(\mathbb{R}^{d}, \mathbb{R}^{N}\right) \quad \text { for } \quad 0 \leqslant|\alpha| \leqslant k .
$$

Here $\theta$ is chosen as in Theorem 2.8 for some $0<\varepsilon<1$. By definition, $u \in W_{\theta}^{k, p}\left(\mathbb{R}^{d}, \mathbb{R}^{N}\right)$ implies $D^{\alpha} u \in W_{\theta}^{k-|\alpha|, p}\left(\mathbb{R}^{d}, \mathbb{R}^{N}\right)$ for every $0 \leqslant|\alpha| \leqslant k$. Since $\theta$ belongs to $C^{\infty}\left(\mathbb{R}^{d}, \mathbb{R}\right)$ and satisfies $\left\|\frac{D^{\gamma} \theta}{\theta}\right\|_{L^{\infty}} \leqslant C(\gamma)$ for every $\gamma \in \mathbb{N}_{0}^{d}$, we obtain $\theta D^{\alpha} u \in W^{k-|\alpha|, p}\left(\mathbb{R}^{d}, \mathbb{R}^{N}\right)$ from

$$
\begin{aligned}
& \left\|\theta D^{\alpha} u\right\|_{W^{k-|\alpha|, p}}^{p}=\sum_{|\beta| \leqslant k-|\alpha|}\left\|D^{\beta}\left(\theta D^{\alpha} u\right)\right\|_{L^{p}}^{p} \\
= & \sum_{|\beta| \leqslant k-|\alpha|}\left\|\sum_{|\gamma| \leqslant|\beta|}\left(\begin{array}{l}
\beta \\
\gamma
\end{array}\right)\left(D^{\gamma} \theta\right)\left(D^{\alpha+\beta-\gamma} u\right)\right\|_{L^{p}}^{p} \\
\leqslant & \sum_{|\beta| \leqslant k-|\alpha|}\left(\sum_{|\gamma| \leqslant|\beta|}\left(\begin{array}{c}
\beta \\
\gamma
\end{array}\right)\left\|\frac{D^{\gamma} \theta}{\theta}\right\|_{L^{\infty}}\left\|\theta D^{\alpha+\beta-\gamma} u\right\|_{L^{p}}\right)^{p} \leqslant C\|u\|_{W_{\theta}^{k, p}}^{p} .
\end{aligned}
$$

Finally, recall the generalized Hölder's inequality

$$
\left\|\prod_{j=1}^{\ell} u_{j}\right\|_{L^{p}\left(\mathbb{R}^{d}, \mathbb{R}\right)} \leq \prod_{j=1}^{\ell}\left\|u_{j}\right\|_{L^{p_{j}\left(\mathbb{R}^{d}, \mathbb{R}\right)},}
$$

for $u_{j} \in L^{p_{j}}\left(\mathbb{R}^{d}, \mathbb{R}\right), 1 \leqslant p, p_{j} \leqslant \infty$ and $\sum_{j=1}^{\ell} \frac{1}{p_{j}}=\frac{1}{p}$.

The following corollary shows, that if we assume more regularity for the solution $v_{\star}$ of $(2.8)$ in Theorem 2.8, i.e. $v_{\star} \in C^{3}\left(\mathbb{R}^{d}, \mathbb{R}^{N}\right)$, then $v_{\star}$ even belongs to 
$W_{\theta}^{2, p}\left(\mathbb{R}^{d}, \mathbb{R}^{N}\right)$. The proof is based on the results of Theorem 2.8 and on a further application of Theorem 3.5. For the proof it is crucial that we allow inhomogeneities $g$ in Theorem 3.5. The argument can be continued to higher order weighted Sobolev spaces.

Corollary 4.1 (Exponential decay of $v_{\star}$ with higher regularity). Let the assumptions (A4), (A6)-(A9) and (A11) be satisfied for $\mathbb{K}=\mathbb{R}$ and for some $1<p<$ $\infty$. Moreover, let $a_{\max }=\rho(A)$ denote the spectral radius of $A,-a_{0}=s(-A)$ the spectral bound of $-A$ and $-b_{0}=s\left(D f\left(v_{\infty}\right)\right)$ the spectral bound of $D f\left(v_{\infty}\right)$. Further, let $\theta(x)=\exp \left(\mu \sqrt{|x|^{2}+1}\right)$ denote a weight function for some $\mu \in \mathbb{R}$. Then, for every $0<\varepsilon<1$ there is a constant $K_{1}=K_{1}\left(A, f, v_{\infty}, d, p, \varepsilon\right)>0$ with the following property: Every classical solution $v_{\star}$ of

$$
A \triangle v(x)+\langle S x, \nabla v(x)\rangle+f(v(x))=0, x \in \mathbb{R}^{d},
$$

with $v_{\star} \in C^{3}\left(\mathbb{R}^{d}, \mathbb{R}^{N}\right)$ and

$$
\sup _{|x| \geqslant R_{0}}\left|v_{\star}(x)-v_{\infty}\right| \leqslant K_{1} \text { for some } R_{0}>0
$$

satisfies

$$
v_{\star}-v_{\infty} \in W_{\theta}^{2, p}\left(\mathbb{R}^{d}, \mathbb{R}^{N}\right)
$$

for every exponential decay rate

$$
0 \leqslant \mu \leqslant \varepsilon \frac{\sqrt{a_{0} b_{0}}}{a_{\max } p} .
$$

If additionally, $p \geqslant \frac{d}{2}, f \in C^{k-1}\left(\mathbb{R}^{N}, \mathbb{R}^{N}\right)$, and $v_{\star} \in C^{k+1}\left(\mathbb{R}^{d}, \mathbb{R}^{N}\right)$ for some $k \in \mathbb{N}$ with $k \geqslant 3$, then $v_{\star}$ even satisfies $v_{\star}-v_{\infty} \in W_{\theta}^{k, p}\left(\mathbb{R}^{d}, \mathbb{R}^{N}\right)$.

Proof. Let $v_{\star}$ be a classical solution of (4.6) satisfying (4.7). Again this implies $v_{\star} \in C_{\mathrm{b}}\left(\mathbb{R}^{d}, \mathbb{R}^{N}\right)$.

1. The additional regularity $v_{\star} \in C^{3}\left(\mathbb{R}^{d}, \mathbb{R}^{N}\right)$ allows us to apply $D_{i}=\frac{\partial}{\partial x_{i}}$ to equation (4.6)

$$
0=A \triangle D_{i} v_{\star}(x)+\left\langle S x, \nabla D_{i} v_{\star}(x)\right\rangle+D f\left(v_{\star}(x)\right) D_{i} v_{\star}(x)+\sum_{j=1}^{d} S_{j i} D_{j} v_{\star}, x \in \mathbb{R}^{d} .
$$

For $w_{\star}:=D_{i} v_{\star} \in C^{2}\left(\mathbb{R}^{d}, \mathbb{R}^{N}\right)$ we obtain using $S_{i i}=0$,

$$
\begin{aligned}
0 & =A \triangle w_{\star}(x)+\left\langle S x, \nabla w_{\star}(x)\right\rangle+D f\left(v_{\star}(x)\right) w_{\star}(x)+\sum_{\substack{j=1 \\
j \neq i}}^{d} S_{j i} D_{j} v_{\star} \\
& =\left[\mathcal{L}_{Q} w_{\star}\right](x)+g(x), x \in \mathbb{R}^{d}
\end{aligned}
$$

with the settings

$$
B_{\infty}:=-D f\left(v_{\infty}\right), Q(x):=D f\left(v_{\star}(x)\right)-D f\left(v_{\infty}\right), g(x):=\sum_{\substack{j=1 \\ j \neq i}}^{d} S_{j i} D_{j} v_{\star} .
$$


2. We now apply Theorem 3.5 with $B_{\infty}, Q, g$ from (4.8), $\theta_{2}=\theta, \mu_{2}=\mu, \mu_{1}<0$ and $\lambda=0$. The assumptions (A4) and (A6) are directly satisfied while $\left(\mathrm{A} 9_{B_{\infty}}\right)$, $\left(\mathrm{A} 11_{B_{\infty}}\right)$ follow from (A9), (A11). In the following let $0<\varepsilon<1$ be fixed and let $\theta_{1}, \theta_{2} \in C\left(\mathbb{R}^{d}, \mathbb{R}\right)$ be given by (3.19) satisfying $\mu_{1}<0 \leqslant \mu_{2}$ and (3.20). The relation $w_{\star} \in W_{\text {loc }}^{2, p}\left(\mathbb{R}^{d}, \mathbb{C}^{N}\right) \cap L_{\theta_{1}}^{p}\left(\mathbb{R}^{d}, \mathbb{C}^{N}\right)$ is a consequence of Theorem 2.8 which implies $D_{i} v_{\star} \in L_{\theta}^{p}\left(\mathbb{R}^{d}, \mathbb{R}^{N}\right) \subseteq L_{\theta_{1}}^{p}\left(\mathbb{R}^{d}, \mathbb{R}^{N}\right)$ since $\theta_{1}$ is decreasing. Moreover, $-\mathcal{L}_{Q} w_{\star}=g$ holds in $L_{\text {loc }}^{p}\left(\mathbb{R}^{d}, \mathbb{C}^{N}\right)$ by construction. Then, $Q \in L^{\infty}\left(\mathbb{R}^{d}, \mathbb{C}^{N, N}\right)$ follows from (4.8) since $v_{\star}$ is bounded, and we also have $g \in L_{\theta}^{p}\left(\mathbb{R}^{d}, \mathbb{C}^{N}\right)$ since Theorem 2.8 shows $v_{\star} \in W_{\theta}^{1, p}\left(\mathbb{R}^{d}, \mathbb{R}^{N}\right)$ which leads to the estimate

$$
\|g\|_{L_{\theta}^{p}} \leqslant \sum_{\substack{j=1 \\ j \neq i}}^{d}\left|S_{j i}\right|\left\|D_{j} v_{\star}\right\|_{L_{\theta}^{p}} \leqslant C .
$$

3. We now verify condition (3.21): Let us choose $K_{1}=K_{1}\left(A, f, v_{\infty}, d, p, \varepsilon\right)>0$ such that (4.2) holds with $\frac{K(\varepsilon)}{2}$ instead of $K(\varepsilon)$ on the right-hand side. Since $f \in C^{2}\left(\mathbb{R}^{N}, \mathbb{R}^{N}\right)$ by (A7), equations (2.9) and (4.2) imply for all $|x| \geqslant R_{0}$

$$
\begin{aligned}
|Q(x)| & =\left|D f\left(v_{\star}(x)\right)-D f\left(v_{\infty}\right)\right| \\
& \leqslant \int_{0}^{1} \mid D^{2} f\left(v_{\infty}+s\left(v_{\star}(x)-v_{\infty}\right)|d s| v_{\star}(x)-v_{\infty} \mid\right. \\
& \leqslant K_{1}\left(\sup _{z \in B_{K_{1}}\left(v_{\infty}\right)}\left|D^{2} f(z)\right|\right) \leqslant \frac{\varepsilon}{2} \min \left\{\frac{b_{0}}{\kappa a_{1}}, \frac{b_{0}}{C_{0, \varepsilon}}, \beta_{\infty}\right\} .
\end{aligned}
$$

Taking the supremum over $|x| \geqslant R_{0}$ we have shown (3.21) with $R_{0}$ from (2.9). By applying Theorem 3.5 we obtain $w_{\star}=D_{i} v_{\star} \in W_{\theta}^{1, p}\left(\mathbb{R}^{d}, \mathbb{R}^{N}\right)$ for every $i=$ $1, \ldots, d$, thus $v_{\star}-v_{\infty} \in W_{\theta}^{2, p}\left(\mathbb{R}^{d}, \mathbb{R}^{N}\right)$.

4. For the final assertion we consider $f \in C^{k-1}\left(\mathbb{R}^{N}, \mathbb{R}^{N}\right)$ and $v_{\star} \in C^{k+1}\left(\mathbb{R}^{d}, \mathbb{R}^{N}\right)$ for some $k \in \mathbb{N}$ with $k \geqslant 3$ and show $v_{\star}-v_{\infty} \in W_{\theta}^{k, p}\left(\mathbb{R}^{d}, \mathbb{R}^{N}\right)$ by induction with respect to $k$. Let $\alpha \in \mathbb{N}_{0}^{d}$ be a multi-index of length $|\alpha|=k-1$ for some $k \geqslant 3$. Applying $D^{\alpha}$ to (4.6) yields that $w_{\star}:=D^{\alpha} v_{\star}$ satisfies

$$
0=\left[\mathcal{L}_{Q} w_{\star}\right](x)+g(x), x \in \mathbb{R}^{d}
$$

with $B_{\infty}$ and $Q(x)$ as in (4.8), and $g(x):=g_{1}(x)+g_{2}(x)$ defined by

$$
\begin{aligned}
g_{1}(x) & :=\sum_{i=1}^{d} \sum_{\substack{j=1 \\
e_{j} \leqslant \alpha}}^{d} S_{i j}\left(\begin{array}{c}
\alpha \\
e_{j}
\end{array}\right) D^{\alpha-e_{j}+e i} v_{\star}(x), \\
g_{2}(x) & :=\sum_{\ell=2}^{k-1} \sum_{\pi \in \mathcal{P}_{\ell, k-1}}\left(D^{\ell} f\right)\left(v_{\star}(x)\right)\left[D^{\left|\pi_{1}\right|} v_{\star}(x) h_{\pi_{1}}, \ldots, D^{\left|\pi_{\ell}\right|} v_{\star}(x) h_{\pi_{\ell}}\right] .
\end{aligned}
$$


The first term $g_{1}$ arises from the Leibniz rule applied to $\left\langle S x, \nabla v_{\star}(x)\right\rangle$,

$$
\begin{aligned}
& D^{\alpha}\left(\left\langle S x, \nabla v_{\star}(x)\right\rangle\right)=\sum_{i=1}^{d} \sum_{j=1}^{d} S_{i j} D^{\alpha}\left(x_{j} D_{i} v_{\star}(x)\right) \\
= & \left\langle S x, \nabla D^{\alpha} v_{\star}(x)\right\rangle+\sum_{i=1}^{d} \sum_{\substack{j=1 \\
e_{j} \leqslant \alpha}}^{d} S_{i j}\left(\begin{array}{c}
\alpha \\
e_{j}
\end{array}\right) D^{\alpha-e_{j}+e_{i}} v_{\star}(x) \\
= & \left\langle S x, \nabla D^{\alpha} v_{\star}(x)\right\rangle+g_{1}(x) .
\end{aligned}
$$

The second term is obtained by applying Faá di Bruno's formula for multivariate calculus,

$$
\begin{aligned}
& D^{\alpha}\left(f\left(v_{\star}(x)\right)\right)=\sum_{\ell=1}^{k-1} \sum_{\pi \in \mathcal{P}_{\ell, k-1}}\left(D^{\ell} f\right)\left(v_{\star}(x)\right)\left[D^{|\pi|} v_{\star}(x) h_{\pi}\right] \\
= & D f\left(v_{\star}(x)\right) D^{\alpha} v_{\star}(x)+\sum_{\ell=2}^{k-1} \sum_{\pi \in \mathcal{P}_{\ell, k-1}}\left(D^{\ell} f\right)\left(v_{\star}(x)\right)\left[D^{|\pi|} v_{\star}(x) h_{\pi}\right] \\
= & D f\left(v_{\star}(x)\right) D^{\alpha} v_{\star}(x)+g_{2}(x), x \in \mathbb{R}^{d} .
\end{aligned}
$$

Here $\mathcal{P}_{\ell, k-1}$ denotes the set of all $\ell$-partitions of the set $\langle k-1\rangle=\{1, \ldots, k-1\}$, given by

$$
\mathcal{P}_{\ell, k-1}=\left\{\pi=\left\{\pi_{1}, \ldots, \pi_{\ell}\right\} \subset 2^{\langle k-1\rangle}: \bigcup_{j=1}^{\ell} \pi_{j}=\langle k-1\rangle, \pi_{i} \cap \pi_{j}=\emptyset \forall i \neq j\right\} .
$$

Moreover, we used short-hands for multilinear terms

$$
\begin{gathered}
\left(h_{1}, \ldots, h_{k-1}\right)=(\underbrace{e_{1}, \ldots, e_{1}}_{\alpha_{1}}, \ldots, \underbrace{e_{d}, \ldots, e_{d}}_{\alpha_{d}}), e_{j} j \text {-th unit vector in } \mathbb{R}^{d}, \\
h_{\rho}=\left(h_{\rho_{1}}, \ldots h_{\rho_{\nu}}\right) \text { for index sets } \rho=\left\{\rho_{1}, \ldots \rho_{\nu}\right\} \subseteq\langle k-1\rangle, \\
{\left[D^{|\pi|} v_{\star}(x) h_{\pi}\right]=\left[D^{\left|\pi_{1}\right|} v_{\star}(x) h_{\pi_{1}}, \ldots, D^{\left|\pi_{\ell}\right|} v_{\star}(x) h_{\pi_{\ell}}\right], \pi=\left\{\pi_{1}, \ldots, \pi_{\ell}\right\} \subset 2^{\langle k-1\rangle} .}
\end{gathered}
$$

For $\ell=1$ the only partition is $\pi_{1}=\{\langle k-1\rangle\}$ and $D f\left(v_{\star}\right)\left[D^{k-1} v_{\star} h_{\pi_{1}}\right]$ agrees with $D f\left(v_{\star}\right) D^{\alpha} v_{\star}$. Below we show $g \in L_{\theta}^{p}\left(\mathbb{R}^{d}, \mathbb{R}^{N}\right)$. Then Theorem 3.5 applies to (4.9) and yields $w_{\star}=D^{\alpha} v_{\star} \in W_{\theta}^{1, p}\left(\mathbb{R}^{d}, \mathbb{R}^{N}\right)$ and thus our assertion $v_{\star}-v_{\infty} \in$ $W_{\theta}^{k, p}\left(\mathbb{R}^{d}, \mathbb{R}^{N}\right)$.

5. To verify $g \in L_{\theta}^{p}\left(\mathbb{R}^{d}, \mathbb{R}^{N}\right)$, consider first $g_{1}$. By the first part of the Corollary (base case $k=3$ ) and by the induction hyperthesis (induction step $k>3$ ) we have $v_{\star}-v_{\infty} \in W_{\theta}^{k-1, p}\left(\mathbb{R}^{d}, \mathbb{R}^{N}\right)$. The indices $\gamma:=\alpha-e_{j}+e_{i}$ with $|\alpha|=k-1$ and $e_{j} \leqslant \alpha$ satisfy $|\gamma|=k-1$, hence $D^{\alpha-e_{j}+e_{i}} v_{\star} \in L_{\theta}^{p}\left(\mathbb{R}^{d}, \mathbb{R}^{N}\right)$, and we deduce $g_{1} \in L_{\theta}^{p}\left(\mathbb{R}^{d}, \mathbb{R}^{N}\right)$.

Next we consider $g_{2}$. We show $\theta g_{2} \in L^{p}\left(\mathbb{R}^{d}, \mathbb{R}^{N}\right)$ by using the generalized Hölder's inequality (4.5) with $p_{j}:=\frac{k-1}{\left|\pi_{j}\right|} p$ and $u_{j}:=\theta\left|D^{\left|\pi_{j}\right|} v_{\star} h_{\pi_{j}}\right|$ for $j=$ $1, \ldots, \ell$. Note that $\sum_{j=1}^{\ell} \frac{1}{p_{j}}=\frac{1}{p}$ since $\sum_{j=1}^{\ell}\left|\pi_{j}\right|=k-1$. We obtain

$$
\left\|\theta\left(D^{\ell} f\right)\left(v_{\star}\right)\left[D^{\left|\pi_{1}\right|} v_{\star} h_{\pi_{1}}, \ldots, D^{\left|\pi_{\ell}\right|} v_{\star} h_{\pi_{\ell}}\right]\right\|_{L^{p}}
$$




$$
\begin{aligned}
& \leqslant\left\|\left(D^{\ell} f\right)\left(v_{\star}\right)\right\|_{L^{\infty}}\left\|\prod_{j=1}^{\ell} \theta^{\frac{1}{j}}\left|D^{\left|\pi_{j}\right|} v_{\star} h_{\pi_{j}}\right|\right\|_{L^{p}} \\
& \leqslant\left\|\left(D^{\ell} f\right)\left(v_{\star}\right)\right\|_{L^{\infty}} \prod_{j=1}^{\ell}\left\|\theta^{\frac{1}{j}} D^{\left|\pi_{j}\right|} v_{\star} h_{\pi_{j}}\right\|_{L^{p_{j}}} \\
& \leqslant\left\|\left(D^{\ell} f\right)\left(v_{\star}\right)\right\|_{L^{\infty}} \prod_{j=1}^{\ell}\left\|\theta D^{\left|\pi_{j}\right|} v_{\star} h_{\pi_{j}}\right\|_{L^{p_{j}}},
\end{aligned}
$$

where we used $\theta(x) \geqslant 1$ and $\theta^{\frac{1}{j}}(x) \leqslant \theta(x)$. Note that $v_{\star} \in C_{\mathrm{b}}\left(\mathbb{R}^{d}, \mathbb{R}^{N}\right), f \in$ $C^{k-1}\left(\mathbb{R}^{N}, \mathbb{R}^{N}\right)$ and $\ell \leqslant k-1$ imply the total derivative $\left(D^{\ell} f\right)\left(v_{\star}\right)$ to be bounded on $\mathbb{R}^{d}$. It remains to verify that $\theta D^{\left|\pi_{j}\right|} v_{\star} h_{\pi_{j}} \in L^{p_{j}}\left(\mathbb{R}^{d}, \mathbb{R}^{N}\right)$ : From $v_{\star}-v_{\infty} \in$ $W_{\theta}^{k-1, p}\left(\mathbb{R}^{d}, \mathbb{R}^{N}\right),(4.4)$ we infer $\theta D^{\gamma}\left(v_{\star}-v_{\infty}\right) \in W^{k-1-|\gamma|, p}\left(\mathbb{R}^{d}, \mathbb{R}^{N}\right)$ for all $0 \leqslant$ $|\gamma| \leqslant k-1$. Using $1 \leqslant\left|\pi_{j}\right| \leqslant k-2$ this proves the first assertion in

$$
\theta D^{\left|\pi_{j}\right|} v_{\star} h_{\pi_{j}} \in W^{k-1-\left|\pi_{j}\right|, p}\left(\mathbb{R}^{d}, \mathbb{R}^{N}\right) \subset L^{p_{j}}\left(\mathbb{R}^{d}, \mathbb{R}^{N}\right) .
$$

The second assertion ' $\subset$ ' follows from the Sobolev embedding (4.3), provided that $1<p<p_{j}<\infty$ and $\frac{d}{p}-\left(k-1-\left|\pi_{j}\right|\right) \leqslant \frac{d}{p_{j}}$. The first inequality is implied by $1 \leqslant\left|\pi_{j}\right| \leqslant k-2$,

$$
1<p<\frac{k-1}{k-2} p \leqslant \frac{k-1}{\left|\pi_{j}\right|} p=p_{j}<\infty,
$$

while the second is implied by our assumptions $p \geqslant \frac{d}{2}$ and $k \geqslant 3$,

$$
\frac{d}{p}-\frac{d}{p_{j}}=\frac{d}{p}\left(1-\frac{\left|\pi_{j}\right|}{k-1}\right) \leqslant 2\left(1-\frac{\left|\pi_{j}\right|}{k-1}\right) \leqslant(k-1)\left(1-\frac{\left|\pi_{j}\right|}{k-1}\right)=k-1-\left|\pi_{j}\right| .
$$

REMARK 4.2 (Higher regularity of the profile $v_{\star}$ ). Collecting the results of Theorem 2.8 and Corollary 4.1, we obtain

$$
f \in C^{\max \{2, k-1\}}\left(\mathbb{R}^{N}, \mathbb{R}^{N}\right), v_{\star} \in C^{k+1}\left(\mathbb{R}^{d}, \mathbb{R}^{N}\right) \Longrightarrow v_{\star}-v_{\infty} \in W_{\theta}^{k, p}\left(\mathbb{R}^{d}, \mathbb{R}^{N}\right)
$$

for any $k \in \mathbb{N}$, provided that $1<p<\infty$ from (A4) satisfies $p \geqslant \frac{d}{2}$ if $k \geqslant 3$.

4.3. Pointwise exponential decay. For the pointwise estimates we use the embedding in $L^{\infty}$. The particular choice $q=\infty$ and $l=0$ in (4.4) leads to

$$
W^{k, p}\left(\mathbb{R}^{d}\right) \subseteq L^{\infty}\left(\mathbb{R}^{d}\right), \quad k \geqslant 0,1<p<\infty, d<k p
$$

and to the inequality

$$
\|u\|_{L^{\infty}\left(\mathbb{R}^{d}\right)} \leqslant C_{p, \infty, k, 0}\|u\|_{W^{k, p}\left(\mathbb{R}^{d}\right)} \forall u \in W^{k, p}\left(\mathbb{R}^{d}\right) .
$$

Corollary 4.3 (Pointwise exponentially decaying estimates). Let the assumptions of Corollary 4.1 be satisfied. Moreover, let $f \in C^{\max \{2, k-1\}}\left(\mathbb{R}^{N}, \mathbb{R}^{N}\right), v_{\star} \in$ $C^{k+1}\left(\mathbb{R}^{d}, \mathbb{R}^{N}\right)$ for some $k \in \mathbb{N}$ and let $p \geqslant \frac{d}{2}$ if $k \geqslant 3$. Then the function $v_{\star}-v_{\infty} \in W_{\theta}^{k, p}\left(\mathbb{R}^{d}, \mathbb{R}^{N}\right)$ satisfies the following estimate

$$
\left|D^{\alpha}\left(v_{\star}(x)-v_{\infty}\right)\right| \leqslant C \exp \left(-\mu \sqrt{|x|^{2}+1}\right) \quad \forall x \in \mathbb{R}^{d}
$$

for every exponential decay rate $0 \leqslant \mu \leqslant \varepsilon \frac{\sqrt{a_{0} b_{0}}}{a_{\max } p}$ and for every multi-index $\alpha \in \mathbb{N}_{0}^{d}$ satisfying $d<(k-|\alpha|) p$. 
Proof. The proof follows from (4.4) and the Sobolev embedding (4.14).

REMARK 4.4. In case of $d \in\{2,3\}$ and $p=2$ it is sufficient to choose $k=4$ to obtain pointwise estimates for $D^{\alpha} v_{\star}$ of order $0 \leqslant|\alpha| \leqslant 2$. This requires to assume $f \in C^{3}\left(\mathbb{R}^{N}, \mathbb{R}^{N}\right)$ and $v_{\star} \in C^{5}\left(\mathbb{R}^{d}, \mathbb{R}^{N}\right)$. Note that the authors of [5] consider the case $d=p=2$ and assume $f \in C^{4}\left(\mathbb{R}^{N}, \mathbb{R}^{N}\right)$ for their stability analysis of rotating patterns. Our results show that $f \in C^{3}\left(\mathbb{R}^{N}, \mathbb{R}^{N}\right)$ is sufficient to guarantee [5, Ass.1] which, therefore, can be omitted.

4.4. Application to complex-valued systems. The next corollary extends the results from Theorem 2.8, Corollary 4.1 and Corollary 4.3 to complex-valued systems of type (2.5) which appear in several applications.

Corollary 4.5 (Exponential decay of $v_{\star}$ for $\mathbb{K}=\mathbb{C}$ ). Let the assumptions (A4) and (A6) be satisfied for $\mathbb{K}=\mathbb{C}$ and for some $1<p<\infty$. Moreover, let $g: \mathbb{R} \rightarrow \mathbb{C}^{N, N}$ satisfy the following properties

$\left(\mathrm{A} 7_{g}\right) \quad g \in C^{2}\left(\mathbb{R}, \mathbb{C}^{N, N}\right)$,

$\left(\mathrm{A} 9_{g}\right) \quad A, g(0) \in \mathbb{C}^{N, N}$ are simultaneously diagonalizable (over $\mathbb{C}$ ),

$\left(\mathrm{A} 11_{g}\right)$ There exists $\beta_{\infty}>0$ such that for all $w \in \mathbb{C}^{N}$ with $|w|=1$

$$
\operatorname{Re}\langle w,-g(0) w\rangle \geqslant \beta_{\infty},
$$

and define

$$
f: \mathbb{C}^{N} \rightarrow \mathbb{C}^{N}, \quad f(u)=g\left(|u|^{2}\right) u .
$$

Further, let $a_{\max }=\rho(A)$ denote the spectral radius of $A,-a_{0}=s(-A)$ the spectral bound of $-A,-b_{0}=s(g(0))$ the spectral bound of $g(0)$ and let $\theta(x)=\exp \left(\mu \sqrt{|x|^{2}+1}\right)$ a weight function with $\mu \in \mathbb{R}$. Then, for every $0<\varepsilon<1$ there is a constant $K_{1}=K_{1}(A, g, d, p, \varepsilon)>0$ with the following property: Every classical solution $v_{\star} \in C^{k}\left(\mathbb{R}^{d}, \mathbb{C}^{N}\right)$ of

$$
A \triangle v(x)+\langle S x, \nabla v(x)\rangle+f(v(x))=0, x \in \mathbb{R}^{d},
$$

such that $g \in C^{\max \{2, k-1\}}\left(\mathbb{R}, \mathbb{C}^{N, N}\right)$ for some $k \in \mathbb{N}, p \geqslant \frac{d}{2}$ if $k \geqslant 3$, and

$$
\sup _{|x| \geqslant R_{0}}\left|v_{\star}(x)\right| \leqslant K_{1} \text { for some } R_{0}>0,
$$

satisfies

$$
v_{\star} \in W_{\theta}^{k, p}\left(\mathbb{R}^{d}, \mathbb{C}^{N}\right)
$$

for every exponential decay rate

$$
0 \leqslant \mu \leqslant \varepsilon \frac{\sqrt{a_{0} b_{0}}}{a_{\max } p} .
$$

Moreover, $v_{\star}$ satisfies the following pointwise estimate

$$
\left|D^{\alpha} v_{\star}(x)\right| \leqslant C \exp \left(-\mu \sqrt{|x|^{2}+1}\right) \quad \forall x \in \mathbb{R}^{d}
$$

for every exponential decay rate $0 \leqslant \mu \leqslant \varepsilon \frac{\sqrt{a_{0} b_{0}}}{a_{\max } p}$ and for every multi-index $\alpha \in \mathbb{N}_{0}^{d}$ satisfying $d<(k-|\alpha|) p$. 
Proof. We transform the $N$-dimensional complex-valued system (4.18) into the $2 N$-dimensional real-valued system

$$
\mathbf{A} \triangle \mathbf{v}(x)+\langle S x, \nabla \mathbf{v}(x)\rangle+\mathbf{f}(\mathbf{v}(x))=0, x \in \mathbb{R}^{d},
$$

For this purpose, we decompose $A=A_{1}+i A_{2}$ with $A_{1}, A_{2} \in \mathbb{R}^{N, N}, v=v_{1}+i v_{2}$ with $v_{1}, v_{2}: \mathbb{R}^{d} \rightarrow \mathbb{R}^{N}, f_{1}, f_{2}: \mathbb{R}^{2 N} \rightarrow \mathbb{R}^{N}$ with $f_{1}\left(u_{1}, u_{2}\right)=\operatorname{Re} f\left(u_{1}+i u_{2}\right)$, $f_{2}\left(u_{1}, u_{2}\right)=\operatorname{Im} f\left(u_{1}+i u_{2}\right), g=g_{1}+i g_{2}$ with $g_{1}, g_{2}: \mathbb{R} \rightarrow \mathbb{R}^{N, N}$. Moreover, we define $\mathbf{A} \in \mathbb{R}^{2 N, 2 N}, \mathbf{v} \in \mathbb{R}^{2 N}$ and $\mathbf{f}: \mathbb{R}^{2 N} \rightarrow \mathbb{R}^{2 N}$ by

$$
\mathbf{A}:=\left(\begin{array}{cc}
A_{1} & -A_{2} \\
A_{2} & A_{1}
\end{array}\right), \mathbf{v}:=\left(\begin{array}{c}
v_{1} \\
v_{2}
\end{array}\right), \mathbf{f}(\mathbf{v}):=\left(\begin{array}{c}
f_{1}(\mathbf{v}) \\
f_{2}(\mathbf{v})
\end{array}\right)=\left(\begin{array}{cc}
g_{1}\left(|\mathbf{v}|^{2}\right) & -g_{2}\left(|\mathbf{v}|^{2}\right) \\
g_{2}\left(|\mathbf{v}|^{2}\right) & g_{1}\left(|\mathbf{v}|^{2}\right)
\end{array}\right) \mathbf{v} .
$$

Let us apply Theorem 2.8 to the $2 N$-dimensional problem (4.20) and check its assumptions. First, we collect the following relations of $A$ and $\mathbf{A}$ :

$$
\begin{aligned}
& \lambda \in \sigma(A) \quad \Longleftrightarrow \quad \lambda, \bar{\lambda} \in \sigma(\mathbf{A}), \\
& Y^{-1} A Y=\Lambda_{A} \quad \Longleftrightarrow \quad\left(\begin{array}{cc}
i Y & \bar{Y} \\
Y & -\overline{i Y}
\end{array}\right)^{-1} \mathbf{A}\left(\begin{array}{cc}
i Y & \bar{Y} \\
Y & -\overline{i Y}
\end{array}\right)=\left(\begin{array}{cc}
\Lambda_{A} & 0 \\
0 & \overline{\Lambda_{A}}
\end{array}\right), \\
& \operatorname{Re}\langle v, A v\rangle=\langle\mathbf{v}, \mathbf{A v}\rangle,|v|=|\mathbf{v}|,|A v|=|\mathbf{A v}| .
\end{aligned}
$$

Since $A$ satisfies (A4) for some $1<p<\infty$ and $\mathbb{K}=\mathbb{C}$, we deduce from (4.23), that $\mathbf{A}$ satisfies (A4) for the same $1<p<\infty$ and $\mathbb{K}=\mathbb{R}$. In particular, we have $\gamma_{A}=\gamma_{\mathbf{A}}$ in (A4). Note that if $A$ satisfies (A1), (A2), (A3) for $\mathbb{K}=\mathbb{C}$ then A satisfies (A1), (A2), (A3) for $\mathbb{K}=\mathbb{R}$, as follows from (4.22), (4.21), (4.23). Assumption (A6) is not affected by the transformation. From $\left(\mathrm{A} 7_{g}\right)$ we deduce that $\mathbf{f} \in C^{2}\left(\mathbb{R}^{2 N}, \mathbb{R}^{2 N}\right)$, so that assumption (A7) is satisfied for $\mathbb{K}=\mathbb{R}$. Obviously, $\mathbf{f}\left(v_{\infty}\right)=0$ holds for $v_{\infty}=0 \in \mathbb{R}^{2 N}$, so that condition (A8) is satisfied. Since $A$ and $g(0)$ are simultaneously diagonalizable (over $\mathbb{C}$ ), cf. $\left(\mathrm{A} 9_{g}\right)$, we deduce from $(4.22)$ that $\mathbf{A}$ and

$$
D \mathbf{f}(0)=\left(\begin{array}{cc}
g_{1}(0) & -g_{2}(0) \\
g_{2}(0) & g_{1}(0)
\end{array}\right)
$$

are simultaneously diagonalizable (over $\mathbb{C}$ ). This proves assumption (A9) for $\mathbb{K}=\mathbb{R}$. Finally, $\left(\mathrm{A} 11_{g}\right)$ implies $(\mathrm{A} 11)$ with $\beta_{\infty}$ given by $\left(\mathrm{A} 11_{g}\right)$. Every classical solution $v_{\star}$ of (4.18) satisfying $v_{\star} \in C_{\mathrm{b}}\left(\mathbb{R}^{d}, \mathbb{C}^{N}\right)$ and (4.19) leads to a classical solution

$$
\mathbf{v}_{\star}:=\left(\begin{array}{c}
\operatorname{Re} v_{\star} \\
\operatorname{Im} v_{\star}
\end{array}\right)
$$

of (4.20) satisfying $\mathbf{v}_{\star} \in C_{\mathrm{b}}\left(\mathbb{R}^{d}, \mathbb{R}^{2 N}\right)$ and (4.19). Summarizing, Theorem 2.8 yields $\mathbf{v}_{\star} \in W_{\theta}^{1, p}\left(\mathbb{R}^{d}, \mathbb{R}^{2 N}\right)$, and thus $v_{\star} \in W_{\theta}^{1, p}\left(\mathbb{R}^{d}, \mathbb{C}^{N}\right)$.

In Section 6 we will apply this result to the cubic-quintic complex GinzburgLandau equation which is of the form (4.17). Other examples fitting into this class include the Schrödinger and the Gross-Pitaevskii equation.

\section{Exponential decay of eigenfunctions}

Consider the eigenvalue problem

$$
A \triangle v(x)+\langle S x, \nabla v(x)\rangle+D f\left(v_{\star}(x)\right) v(x)=\lambda v(x), x \in \mathbb{R}^{d}, d \geqslant 2 .
$$

We are interested in classical solutions $(\lambda, v)$ of (5.1), i.e. $\lambda \in \mathbb{C}$ and $v \in$ $C^{2}\left(\mathbb{R}^{d}, \mathbb{C}^{N}\right)$ solves (5.1) pointwise (cf. Definition 2.6). 
5.1. Sobolev and pointwise estimates of eigenfunctions. The following theorem states that every classical solution $v$ of the eigenvalue problem (5.1) decays exponentially in space, provided its associated (isolated) eigenvalue $\lambda$ satisfies $\operatorname{Re} \lambda>-\beta_{\infty}$. The proof is similar to those of Theorem 2.8 and Corollary 4.1, but now it is crucial that Theorem 3.5 can be employed for cases where $\lambda \neq 0, g \neq 0$ and $\mathbb{K}=\mathbb{C}$.

THEOREM 5.1 (Exponential decay of eigenfunctions). (1) Let the assumptions (A4), (A6)-(A9) and (A11) be satisfied for $\mathbb{K}=\mathbb{R}$ and for some $1<p<\infty$. Moreover, let $a_{\max }=\rho(A)$ denote the spectral radius of $A,-a_{0}=s(-A)$ the spectral bound of $-A,-b_{0}=s\left(D f\left(v_{\infty}\right)\right)$ the spectral bound of $D f\left(v_{\infty}\right)$ and let $\beta_{\infty}$ be from (A11). Further, let

$$
\theta_{j}(x)=\exp \left(\mu_{j} \sqrt{|x|^{2}+1}\right), \quad x \in \mathbb{R}^{d}, \quad j=1,2
$$

denote a weight function for $\mu_{1}, \mu_{2} \in \mathbb{R}$. Then, for every $0<\varepsilon<1$ there is a constant $K_{1}=K_{1}\left(A, f, v_{\infty}, d, p, \varepsilon\right)>0$ such that for every classical solution $v_{\star}$ of (2.8) satisfying (2.9) the following property holds: Every classical solution $v$ of the eigenvalue problem

$$
A \triangle v(x)+\langle S x, \nabla v(x)\rangle+D f\left(v_{\star}(x)\right) v(x)=\lambda v(x), x \in \mathbb{R}^{d},
$$

with $\lambda \in \mathbb{C}$ and $\operatorname{Re} \lambda \geqslant-(1-\varepsilon) \beta_{\infty}$, such that $v \in L_{\theta_{1}}^{p}\left(\mathbb{R}^{d}, \mathbb{C}^{N}\right)$ for some exponential growth rate

$$
-\sqrt{\varepsilon \frac{\gamma_{A} \beta_{\infty}}{2 d|A|^{2}}} \leqslant \mu_{1} \leqslant 0
$$

satisfies

$$
v \in W_{\theta_{2}}^{1, p}\left(\mathbb{R}^{d}, \mathbb{C}^{N}\right)
$$

for every exponential decay rate

$$
0 \leqslant \mu_{2} \leqslant \varepsilon \frac{\sqrt{a_{0} b_{0}}}{a_{\max } p} .
$$

(2) If additionally, $p \geqslant \frac{d}{2}, f \in C^{k}\left(\mathbb{R}^{N}, \mathbb{R}^{N}\right)$, $v_{\star} \in C^{k+1}\left(\mathbb{R}^{d}, \mathbb{R}^{N}\right)$ and $v \in C^{k+1}\left(\mathbb{R}^{d}, \mathbb{C}^{N}\right)$ for some $k \in \mathbb{N}$ with $k \geqslant 2$, then $v \in W_{\theta}^{k, p}\left(\mathbb{R}^{d}, \mathbb{C}^{N}\right)$ holds. Moreover, $v$ satisfies the pointwise estimate

$$
\left|D^{\alpha} v(x)\right| \leqslant C \exp \left(-\mu_{2} \sqrt{|x|^{2}+1}\right), \quad x \in \mathbb{R}^{d}
$$

for every exponential decay rate $0 \leqslant \mu_{2} \leqslant \varepsilon \frac{\sqrt{a_{0} b_{0}}}{a_{\max } p}$ and for every multi-index $\alpha \in \mathbb{N}_{0}^{d}$ satisfying $d<(k-|\alpha|) p$.

REMARK 5.2. In case of $d \in\{2,3\}$ and $p=2$ it is sufficient to choose $k=5$ to obtain pointwise estimates for $v$ of order $0 \leqslant|\alpha| \leqslant 2$. This requires to assume $f \in C^{5}\left(\mathbb{R}^{N}, \mathbb{R}^{N}\right), v_{\star} \in C^{6}\left(\mathbb{R}^{d}, \mathbb{R}^{N}\right)$ and $v \in C^{6}\left(\mathbb{R}^{d}, \mathbb{C}^{N}\right)$. Moreover, note that the space $L_{\theta_{1}}^{p}\left(\mathbb{R}^{d}, \mathbb{C}^{N}\right)$ does not only allow bounded but even exponentially growing eigenfunctions.

ProOF. (1) Let $v_{\star}$ be a classical solution of (2.8) satisfying (2.9) and let $v$ be a classical solution of (5.2) satisfying $v \in L_{\theta_{1}}^{p}\left(\mathbb{R}^{d}, \mathbb{C}^{N}\right)$ with $\theta_{1}$ from (3.19) and $\mu_{1}$ such that (5.3). Then $v$ satisfies

$$
0=\lambda v(x)-\left(A \triangle v(x)+\langle S x, \nabla v(x)\rangle-B_{\infty} v(x)+Q(x) v(x)\right)
$$




$$
=\left[\left(\lambda I-\mathcal{L}_{Q}\right) v\right](x), x \in \mathbb{R}^{d}
$$

with $B_{\infty}:=-D f\left(v_{\infty}\right)$ and $Q(x):=D f\left(v_{\star}(x)\right)-D f\left(v_{\infty}\right)$ as in (4.8). Now, $v \in$ $W_{\theta_{2}}^{1, p}\left(\mathbb{R}^{d}, \mathbb{C}^{N}\right)$ follows from Theorem 3.5 with $\mu_{1}<0, g=0$ and $\lambda \in \mathbb{C}$ with $\operatorname{Re} \lambda \geqslant-(1-\varepsilon) \beta_{\infty}$. Note, that the assumptions of Theorem 3.5 are satisfied as shown in the proof of Corollary 4.1.

(2) Assuming more smoothness for $f, v, v_{\star}$, we prove $v \in W_{\theta_{2}}^{k, p}\left(\mathbb{R}^{d}, \mathbb{C}^{N}\right)$ by induction on $k$. Let $\alpha \in \mathbb{N}_{0}^{d}$ be a multi-index of length $|\alpha|=k-1$. Similar to the proof of Corollary 4.1, an application of $D^{\alpha}$ to (5.2) yields an inhomogenous equation for $w:=D^{\alpha} v$,

$$
\left[\left(\lambda I-\mathcal{L}_{Q}\right) w\right](x)=g(x), x \in \mathbb{R}^{d}
$$

with matrices $B_{\infty}$ and $Q(x)$ as in (4.8), $\lambda \in \mathbb{C}$ with $\operatorname{Re} \lambda \geqslant-(1-\varepsilon) \beta_{\infty}$, and $g(x):=g_{1}(x)+g_{2}(x)$ where

$$
\begin{aligned}
g_{1} & :=\sum_{i=1}^{d} \sum_{\substack{j=1 \\
e_{j} \leqslant \alpha}}^{d} S_{i j}\left(\begin{array}{c}
\alpha \\
e_{j}
\end{array}\right) D^{\alpha-e_{j}+e_{i}} v \\
g_{2} & :=\sum_{\substack{\beta \leqslant \alpha \\
|\beta| \geqslant 1}}\left(\begin{array}{c}
\alpha \\
\beta
\end{array}\right) \sum_{\ell=1}^{|\beta|} \sum_{\pi \in \mathcal{P}_{\ell,|\beta|}}\left(D^{\ell+1} f\right)\left(v_{\star}\right)\left[D^{\left|\pi_{1}\right|} v_{\star} h_{\pi_{1}}, \ldots, D^{\left|\pi_{\ell}\right|} v_{\star} h_{\pi_{\ell}}, D^{\alpha-\beta} v\right] .
\end{aligned}
$$

In this expression, the multilinear argument $h$ is defined as in (4.13) with $\alpha$ replaced by $\beta$. Below we prove $g_{1}, g_{2} \in L_{\theta_{2}}^{p}\left(\mathbb{R}^{d}, \mathbb{C}^{N}\right)$, so that Theorem 3.5 implies $w=D^{\alpha} v \in$ $W_{\theta_{2}}^{1, p}\left(\mathbb{R}^{d}, \mathbb{C}^{N}\right)$ and therefore, $v \in W_{\theta_{2}}^{k, p}\left(\mathbb{R}^{d}, \mathbb{C}^{N}\right)$.

First we consider $g_{1}$ : By the first part of this Corollary (base case $k=2$ ) and by the induction hyperthesis (induction step $k>2$ ) we have $v \in W_{\theta_{2}}^{k-1, p}\left(\mathbb{R}^{d}, \mathbb{C}^{N}\right)$. As in the proof of Corollary 4.1, we then deduce $g_{1} \in L_{\theta_{2}}^{p}\left(\mathbb{R}^{d}, \mathbb{R}^{N}\right)$. Finally, we show $g_{2} \in L_{\theta_{2}}^{p}\left(\mathbb{R}^{d}, \mathbb{R}^{N}\right)$ by applying Hölder's inequality (4.5) with $p_{j}:=\frac{k}{\left|\pi_{j}\right|} p$ and $u_{j}:=\theta_{2}^{\frac{1}{j}}\left|D^{\left|\pi_{j}\right|} v_{\star} h_{\pi_{j}}\right|$ for $j=1, \ldots, \ell, p_{\ell+1}:=\frac{k}{k-|\beta|} p$ and $u_{\ell+1}:=\left|D^{\alpha-\beta} v\right|$. Note that $\sum_{j=1}^{\ell+1} \frac{1}{p_{j}}=\frac{1}{p}$ follows from $\sum_{j=1}^{\ell}\left|\pi_{j}\right|=|\beta|$. We obtain

$$
\begin{aligned}
& \left\|\theta_{2}\left(D^{\ell+1} f\right)\left(v_{\star}\right)\left[D^{\left|\pi_{1}\right|} v_{\star} h_{\pi_{1}}, \ldots, D^{\left|\pi_{\ell}\right|} v_{\star} h_{\pi_{\ell}}, D^{\alpha-\beta} v\right]\right\|_{L^{p}} \\
\leqslant & \left\|\left(D^{\ell+1} f\right)\left(v_{\star}\right)\right\|_{L^{\infty}}\left\|\left|D^{\alpha-\beta} v\right| \prod_{j=1}^{\ell} \theta_{2}^{\frac{1}{j}}\left|D^{\left|\pi_{j}\right|} v_{\star} h_{\pi_{j}}\right|\right\|_{L^{p}} \\
\leqslant & \left\|\left(D^{\ell+1} f\right)\left(v_{\star}\right)\right\|_{L^{\infty}}\left\|D^{\alpha-\beta} v\right\|_{L^{p_{\ell+1}}} \prod_{j=1}^{\ell}\left\|\theta_{2}^{\frac{1}{j}} D^{\left|\pi_{j}\right|} v_{\star} h_{\pi_{j}}\right\|_{L^{p_{j}}} \\
\leqslant & \left\|\left(D^{\ell+1} f\right)\left(v_{\star}\right)\right\|_{L^{\infty}}\left\|D^{\alpha-\beta} v\right\|_{L^{p_{\ell+1}}} \prod_{j=1}^{\ell}\left\|\theta_{2} D^{\left|\pi_{j}\right|} v_{\star} h_{\pi_{j}}\right\|_{L^{p_{j}}}
\end{aligned}
$$

since $\theta_{2}(x) \geqslant 1$ and $j \geqslant 1$ imply $\theta_{2}^{\frac{1}{j}}(x) \leqslant \theta_{2}(x)$. Note that $v_{\star} \in C_{\mathrm{b}}\left(\mathbb{R}^{d}, \mathbb{R}^{N}\right)$, $f \in C^{k}\left(\mathbb{R}^{N}, \mathbb{R}^{N}\right)$ and $\ell \leqslant|\beta| \leqslant|\alpha|=k-1$ imply the boundedness of $\left(D^{\ell+1} f\right)\left(v_{\star}\right)$. As in the proof of Corollary 4.1, both $\theta_{2} D^{\left|\pi_{j}\right|} v_{\star} h_{\pi_{j}} \in L^{p_{j}}\left(\mathbb{R}^{d}, \mathbb{R}^{N}\right)$ and $\theta_{2} D^{\alpha-\beta} v \in$ $L^{p_{\ell+1}}\left(\mathbb{R}^{d}, \mathbb{C}^{N}\right)$ follow from the Sobolev embedding (4.3): Since $f \in C^{k-1}\left(\mathbb{R}^{N}, \mathbb{R}^{N}\right)$ 
and $\quad v_{\star} \quad \in \quad C^{k+1}\left(\mathbb{R}^{d}, \mathbb{R}^{N}\right), \quad$ Corollary $4.1 \quad$ implies that $v_{\star}-v_{\infty} \in W_{\theta_{2}}^{k, p}\left(\mathbb{R}^{d}, \mathbb{R}^{N}\right)$, therefore $\theta_{2} D^{\left|\pi_{j}\right|} v_{\star} h_{\pi_{j}} \in W^{k-\left|\pi_{j}\right|, p}\left(\mathbb{R}^{d}, \mathbb{R}^{N}\right)$. The Sobolev embedding (4.3) shows

$$
\theta_{2} D^{\left|\pi_{j}\right|} v_{\star} h_{\pi_{j}} \in W^{k-\left|\pi_{j}\right|, p}\left(\mathbb{R}^{d}, \mathbb{R}^{N}\right) \subseteq L^{p_{j}}\left(\mathbb{R}^{d}, \mathbb{R}^{N}\right) \forall 1 \leqslant\left|\pi_{j}\right| \leqslant|\beta|,
$$

provided that $1<p<p_{j}<\infty$ and $\frac{d}{p}-\left(k-\left|\pi_{j}\right|\right) \leqslant \frac{d}{p_{j}}$. These conditions are obviously satisfied, since $p \geqslant \frac{d}{2}, 1 \leqslant\left|\pi_{j}\right| \leqslant k-1$. Next, since $v \in W_{\theta_{2}}^{k-1, p}\left(\mathbb{R}^{d}, \mathbb{C}^{N}\right)$, (4.4) implies $\theta_{2} D^{\gamma} v \in W^{k-1-|\gamma|, p}\left(\mathbb{R}^{d}, \mathbb{C}^{N}\right)$ for all $0 \leqslant|\gamma| \leqslant k-1$. For $\gamma=\alpha-\beta$ with $|\alpha|=k-1$ and $\beta \leqslant \alpha$ we have $k-1-|\gamma|=|\beta|$ and therefore, the Sobolev embedding (4.3) implies

$$
\theta_{2} D^{\alpha-\beta} v \in W^{|\beta|, p}\left(\mathbb{R}^{d}, \mathbb{R}^{N}\right) \subset L^{p_{\ell+1}}\left(\mathbb{R}^{d}, \mathbb{R}^{N}\right),
$$

provided that $1<p<p_{\ell+1}<\infty$ and $\frac{d}{p}-|\beta| \leqslant \frac{d}{p_{\ell+1}}$. These conditions are satisfied by the same arguments as above. This concludes the proof of $g_{2} \in L_{\theta_{2}}^{p}\left(\mathbb{R}^{d}, \mathbb{R}^{N}\right)$.

Finally, the pointwise estimates follow when combining our previous Sobolev estimates with (4.4) and the embedding (4.14), in a similar manner as in Corollary 4.3

REMARK 5.3 (Higher regularity of the eigenfunction $v$ ). Collecting the results of Theorem 5.1 and Remark 4.2, we obtain

$$
f \in C^{\max \{2, k\}}, v_{\star}, v \in C^{k+1} \Longrightarrow v_{\star}-v_{\infty}, v \in W_{\theta_{2}}^{k, p}
$$

for any $k \in \mathbb{N}$, provided that $1<p<\infty$ from (A4) satisfies $p \geqslant \frac{d}{2}$ if $k \geqslant 2$. We also recall the role of the parameter $\varepsilon$ in the exponential estimates. Theorem 5.1 shows that every eigenfunction associated to an eigenvalue $\lambda$ with $\operatorname{Re} \lambda>-\beta_{\infty}$ decays exponentially in space. Usually, one expects this behavior even for $\operatorname{Re} \lambda>-b_{0}$. The rate of decay is controlled by $\varepsilon \in(0,1)$. If $\operatorname{Re} \lambda \geqslant-(1+\varepsilon) \beta_{\infty}$ is close to $-\beta_{\infty}$ we may take $\varepsilon$ close to 0 and obtain a small rate $\mu_{2}$ of decay according to (5.4). On the other hand, if $\lambda$ is close to the imaginary axis we may take $\varepsilon$ close to 1 and obtain a higher rate of decay.

5.2. Eigenfunctions belonging to eigenvalues on the imaginary axis. Some classical solutions of the eigenvalue problem (5.1) are due to equivariance of the underlying equations and can be expressed in terms of the rotating wave itself. The following result proved in [26, Thm.9.4], specifies these eigenfunctions.

THEOREM 5.4 (Point spectrum on the imaginary axis). Let $S \in \mathbb{R}^{d, d}$ be skewsymmetric and let $U \in \mathbb{C}^{d, d}$ denote the unitary matrix satisfying $\Lambda_{S}=\bar{U}^{T} S U$ with $\Lambda_{S}=\operatorname{diag}\left(\lambda_{1}^{S}, \ldots, \lambda_{d}^{S}\right)$ and eigenvalues $\lambda_{1}^{S}, \ldots, \lambda_{d}^{S}$ of $S$. Moreover, let $v_{\star} \in$ $C^{3}\left(\mathbb{R}^{d}, \mathbb{R}^{N}\right)$ be a classical solution of $(2.8)$, then $v: \mathbb{R}^{d} \rightarrow \mathbb{C}^{N}$ given by

$$
\begin{aligned}
v(x) & =\left\langle C^{\mathrm{rot}} x+C^{\mathrm{tra}}, \nabla v_{\star}(x)\right\rangle \\
& =\sum_{i=1}^{d-1} \sum_{j=i+1}^{d} C_{i j}^{\mathrm{rot}}\left(x_{j} D_{i}-x_{i} D_{j}\right) v_{\star}(x)+\sum_{l=1}^{d} C_{l}^{\mathrm{tra}} D_{l} v_{\star}(x)
\end{aligned}
$$

is a classical solution of the eigenvalue problem (5.1) if $C^{\text {rot }} \in \mathbb{C}^{d, d}, C^{\text {tra }} \in \mathbb{C}^{d}$ either satisfy

$$
\lambda=-\lambda_{l}^{S}, \quad C^{\mathrm{rot}}=0, \quad C^{\mathrm{tra}}=U e_{l}
$$


for some $l=1, \ldots, d$, or

$$
\lambda=-\left(\lambda_{n}^{S}+\lambda_{m}^{S}\right), \quad C^{\mathrm{rot}}=U\left(I_{n m}-I_{m n}\right) U^{T}, \quad C^{\text {tra }}=0
$$

for some $n=1, \ldots, d-1$ and $m=n+1, \ldots, d$. Here $I_{n m} \in \mathbb{R}^{d, d}$ denotes the matrix having the entries 1 at the $n$-th row and $m$-th column and 0 otherwise. All the eigenvalues above lie on the imaginary axis.

A direct consequence of Theorem 5.1 and Theorem 5.4 is that the eigenfunctions $v$ from $(5.7)$ belong to $W_{\theta_{2}}^{1, p}\left(\mathbb{R}^{d}, \mathbb{C}^{N}\right)$ and decay exponentially in space, [26, Thm.9.8].

Corollary 5.5 (Exponential decay of eigenfunctions for eigenvalues on $i \mathbb{R}$ ). Let all assumptions of the statements (1) and (2) of Theorem 5.1 be satisfied. Then the classical solution

$$
v(x)=\left\langle C^{\mathrm{rot}} x+C^{\mathrm{tra}}, \nabla v_{\star}(x)\right\rangle, \quad x \in \mathbb{R}^{d}
$$

of the eigenvalue problem (5.2) with $\lambda, C^{\text {rot }}$ and $C^{\text {tra }}$ from Theorem 5.4 lies in $W_{\theta_{2}}^{k, p}\left(\mathbb{R}^{d}, \mathbb{C}^{N}\right)$ for every exponential decay rate (5.4). Moreover, the function $v \in$ $W_{\theta_{2}}^{k, p}\left(\mathbb{R}^{d}, \mathbb{C}^{N}\right)$ satisfies the pointwise estimate (5.5).

Proof. In order to apply Theorem 5.1 to $v(x)=\left\langle C^{\text {rot }} x+C^{\text {tra }}, \nabla v_{\star}(x)\right\rangle$, we observe that the map $x \mapsto\left\langle C^{\text {rot }} x+C^{\text {tra }}, \nabla v_{\star}(x)\right\rangle$ is of class $C^{k+1}$ since $v_{\star} \in$ $C^{k+2}\left(\mathbb{R}^{d}, \mathbb{R}^{N}\right)$. In particular, $\beta_{\infty}>0$ allows to deal with eigenvalues $\lambda \in i \mathbb{R}$.

REMARK 5.6. Later on, we numerically approximate the spectrum of the linearization $\mathcal{L}$ for (2.12). For this purpose we decompose the $L^{p}$-spectrum $\sigma(\mathcal{L})$ of $\mathcal{L}$ into the disjoint union of point spectrum $\sigma_{\text {point }}(\mathcal{L})$ and essential spectrum $\sigma_{\text {ess }}(\mathcal{L})$

$$
\sigma(\mathcal{L})=\sigma_{\text {point }}(\mathcal{L}) \cup \sigma_{\text {ess }}(\mathcal{L}) .
$$

The point spectrum of $\mathcal{L}$ is affected by the symmetries of the group action and contains the eigenvalues described in Theorem 5.4. In particular, it contains the spectrum of $S$ and the sum of its different eigenvalues, i.e.

$$
\sigma_{\text {point }}^{\text {part }}(\mathcal{L}):=\sigma(S) \cup\left\{\lambda_{1}+\lambda_{2} \mid \lambda_{1}, \lambda_{2} \in \sigma(S), \lambda_{1} \neq \lambda_{2}\right\} . \subseteq \sigma_{\text {point }}(\mathcal{L})
$$

The associated eigenfunctions are explictly known, see (5.7), and they are exponentially localized as shown in Corollary 5.5. In general, $\sigma_{\text {point }}(\mathcal{L})$ contains further eigenvalues. Neither these additional eigenvalues nor their associated eigenfunctions can usually be determined explicitly. The essential spectrum of $\mathcal{L}$ depends on the asymptotic behavior of the wave at infinity. Under the same assumptions as in Theorem 2.8 one derives a dispersion relation for rotating waves, see [26, Sec.9.5],

$$
\operatorname{det}\left(\lambda I_{N}+\omega^{2} A-D f\left(v_{\infty}\right)+i \sum_{l=1}^{m} n_{l} \sigma_{l} I_{N}\right)=0 \text { for some } \omega \in \mathbb{R}, n_{l} \in \mathbb{Z}
$$

which then yields information about the essential spectrum

$$
\sigma_{\mathrm{ess}}^{\text {part }}(\mathcal{L}):=\{\lambda \in \mathbb{C} \mid \lambda \text { satisfies }(5.10)\} \subseteq \sigma_{\mathrm{ess}}(\mathcal{L}) .
$$

Here, $S$ has the $(d-2 m)$-fold eigenvalue 0 and nontrivial eigenvalues $\pm i \sigma_{l}, l=$ $1, \ldots, m$ on the imaginary axis. For more details we refer to the examples in Section 6 and to $[26$, Ch.9,10]. 


\section{Rotating waves in reaction diffusion systems: The cubic-quintic complex Ginzburg-Landau equation}

Consider the cubic-quintic complex Ginzburg-Landau equation (QCGL), [19]

$$
u_{t}=\alpha \Delta u+u\left(\delta+\beta|u|^{2}+\gamma|u|^{4}\right)
$$

where $u: \mathbb{R}^{d} \times[0, \infty) \rightarrow \mathbb{C}, d \in\{2,3\}, \alpha, \beta, \gamma, \delta \in \mathbb{C}$ with $\operatorname{Re} \alpha>0$ and $f: \mathbb{C} \rightarrow \mathbb{C}$ given by

$$
f(u):=u\left(\delta+\beta|u|^{2}+\gamma|u|^{4}\right) .
$$

The real-valued version of (6.1) reads as follows

$$
\mathbf{u}_{t}=\mathbf{A} \triangle \mathbf{u}+\mathbf{f}(\mathbf{u}) \quad \text { with } \quad \mathbf{A}:=\left(\begin{array}{cc}
\alpha_{1} & -\alpha_{2} \\
\alpha_{2} & \alpha_{1}
\end{array}\right), \quad \mathbf{u}=\left(\begin{array}{l}
u_{1} \\
u_{2}
\end{array}\right)
$$

and $\mathbf{f}: \mathbb{R}^{2} \rightarrow \mathbb{R}^{2}$ given by

$$
\mathbf{f}\left(\begin{array}{l}
u_{1} \\
u_{2}
\end{array}\right):=\left(\begin{array}{l}
\left(u_{1} \delta_{1}-u_{2} \delta_{2}\right)+\left(u_{1} \beta_{1}-u_{2} \beta_{2}\right)|\mathbf{u}|^{2}+\left(u_{1} \gamma_{1}-u_{2} \gamma_{2}\right)|\mathbf{u}|^{4} \\
\left(u_{1} \delta_{2}+u_{2} \delta_{1}\right)+\left(u_{1} \beta_{2}+u_{2} \beta_{1}\right)|\mathbf{u}|^{2}+\left(u_{1} \gamma_{2}+u_{2} \gamma_{1}\right)|\mathbf{u}|^{4}
\end{array}\right)
$$

where $|\mathbf{u}|^{2}=u_{1}^{2}+u_{2}^{2}, u=u_{1}+i u_{2}, \alpha=\alpha_{1}+i \alpha_{2}, \beta=\beta_{1}+i \beta_{2}, \gamma=\gamma_{1}+i \gamma_{2}$, $\delta=\delta_{1}+i \delta_{2}$.

This equation describes different aspects of signal propagation in heart tissue, superconductivity, superfluidity, nonlinear optical systems, see [23], photonics, plasmas, physics of lasers, Bose-Einstein condensation, liquid crystals, fluid dynamics, chemical waves, quantum field theory, granular media and is used in the study of hydrodynamic instabilities, see [21]. It shows a variety of coherent structures like stable and unstable pulses, fronts, sources and sinks in $1 \mathrm{D}$, see $[3,36,38,39]$, vortex solitons, see [13], spinning solitons, see [14], dissipative ring solitons, see [33], rotating spiral waves, propagating clusters, see [30], and exploding dissipative solitons, see [34] in 2D as well as scroll waves and spinning solitons in $3 \mathrm{D}$, see [22].

We are interested in exponentially localized rotating wave solutions $u_{\star}: \mathbb{R}^{d} \times[0, \infty) \rightarrow \mathbb{C}$ of $(6.1)$ and $\mathbf{u}_{\star}: \mathbb{R}^{d} \times[0, \infty) \rightarrow \mathbb{R}^{2}$ of $(6.3)$. Note that, given some skew-symmetric $S \in \mathbb{R}^{d, d}$ and some vector $x_{\star} \in \mathbb{R}^{d}$, the function $u_{\star}(x, t)=v_{\star}\left(e^{-t S}\left(x-x_{\star}\right)\right)$ with $v_{\star}: \mathbb{R}^{d} \rightarrow \mathbb{C}$ is a rotating wave of $(6.1)$ if and only if $\mathbf{u}_{\star}(x, t)=\mathbf{v}_{\star}\left(e^{-t S}\left(x-x_{\star}\right)\right)$ is a rotating wave of $(6.3)$, where $\mathbf{u}_{\star}=\left(\begin{array}{l}\operatorname{Re} u_{\star} \\ \operatorname{Im} u_{\star}\end{array}\right)$ and $\mathbf{v}_{\star}=\left(\begin{array}{l}\operatorname{Re} v_{\star} \\ \operatorname{Im} v_{\star}\end{array}\right)$. We are going to show that $v_{\star}$ (and $\mathbf{v}_{\star}$ ) are exponentially localized by applying Theorem 2.8 and Corollaries 4.1, 4.3 to the real-valued system (6.3) and Corollary 4.5 to the complex equation (6.1).

First, consider the assumptions (A1)-(A11) for $\mathbb{K}=\mathbb{R}$ : With A from (6.3) and f from (6.4), Assumption (A1) is satisfied for every $\alpha \in \mathbb{C}$, since

$$
Y^{-1} \mathbf{A} Y=\Lambda_{\mathbf{A}}, \Lambda_{\mathbf{A}}=\left(\begin{array}{cc}
\alpha & 0 \\
0 & \bar{\alpha}
\end{array}\right), Y=\left(\begin{array}{cc}
i & 1 \\
1 & i
\end{array}\right), \quad Y^{-1}=\frac{1}{2}\left(\begin{array}{cc}
-i & 1 \\
1 & -i
\end{array}\right) .
$$

Assumption (A2) follows from $\operatorname{Re} \alpha>0$, since $\sigma(\mathbf{A})=\{\alpha, \bar{\alpha}\}$. Assumption (A3) holds with $\beta_{\mathbf{A}}=\operatorname{Re} \alpha$ if $\operatorname{Re} \alpha>0$, since $\operatorname{Re}\langle w, \mathbf{A} w\rangle=\operatorname{Re} \alpha$ for $w \in \mathbb{R}^{2}$ with $|w|=1$. Condition (A5), which is equivalent to (A4), requires $\alpha \neq 0$ and $\frac{\operatorname{Re} \alpha}{|\alpha|}=\mu_{1}(\mathbf{A})>$ 
$\frac{|p-2|}{p}$. The latter condition is equivalent to

$$
|\arg \alpha|<\arctan \left(\frac{2 \sqrt{p-1}}{|p-2|}\right) \text { for some } 1<p<\infty,
$$

or alternatively to

$$
p_{\min }:=\frac{2|\alpha|}{|\alpha|+\operatorname{Re} \alpha}<p<\frac{2|\alpha|}{|\alpha|-\operatorname{Re} \alpha}=: p_{\max } .
$$

The condition (A6) is satisfied with $S \in \mathbb{R}^{d, d}$ given by

$$
S=\left(\begin{array}{cc}
0 & S_{12} \\
-S_{12} & 0
\end{array}\right) \quad \text { and } \quad S=\left(\begin{array}{ccc}
0 & S_{12} & S_{13} \\
-S_{12} & 0 & S_{23} \\
-S_{13} & -S_{23} & 0
\end{array}\right)
$$

for $d=2$ and $d=3$, respectively. Below we specify the entries $S_{12}, S_{13}, S_{23} \in$ $\mathbb{R}$ and the point $x_{\star} \in \mathbb{R}^{d}$, that will be the center of rotation if $d=2$ and a support vector of the axis of rotation if $d=3$, cf. (2.2). All this information come actually from a simulation. First we simulate the original system for some time. Then we switch to the freezing method, which yields the profile $v_{\star}$, its center of rotation, and its rotational velocities. For more details on the computation, see [26, Sec.10.3]. Some general theory and applications of the freezing method may be found in $[7,8,9,10,37]$. Note that in case $d=2$ we have a clockwise rotation, if $S_{12}>0$, and a counter clockwise rotation, if $S_{12}<0$. Assumption (A7) is obviously satisfied, even with $\mathbf{f} \in C^{\infty}\left(\mathbb{R}^{2}, \mathbb{R}^{2}\right)$, since every component of $\mathbf{f}$ is a polynomial. With $\mathbf{v}_{\infty}=(0,0)^{T}$, the assumption (A8) is satisfied, and for this choice we have

$$
D \mathbf{f}\left(\mathbf{v}_{\infty}\right)=\left(\begin{array}{cc}
\delta_{1} & -\delta_{2} \\
\delta_{2} & \delta_{1}
\end{array}\right)
$$

Assumption (A9) holds for the same transformation matrix $Y$ as in (6.5). The condition $\operatorname{Re} \delta<0$ implies both, Assumption (A10) and Assumption (A11) with $\beta_{\infty}=-\operatorname{Re} \delta$.

Next we consider assumptions $\left(\mathrm{A} 7_{g}\right),\left(\mathrm{A} 9_{g}\right)$ and $\left(\mathrm{A} 11_{g}\right)$ : Writing $f$ as $f(u)=$ $g\left(|u|^{2}\right) u$ with

$$
g: \mathbb{R} \rightarrow \mathbb{C}, \quad g(v)=\delta+\beta v+\gamma v^{2},
$$

Assumption $\left(\mathrm{A} 7_{g}\right)$ is obivously satisfied and we even have $g \in C^{\infty}(\mathbb{R}, \mathbb{C})$. Assumption $\left(\mathrm{A} 9_{g}\right)$ is satisfied with $g(0)=\delta$ for every $\alpha, \delta \in \mathbb{C}$ and assumption $\left(\mathrm{A} 11_{g}\right)$ with $\beta_{\infty}=-\operatorname{Re} \delta$ if $\operatorname{Re} \delta<0$. The assumptions (A1)-(A5) for $A=\alpha \in \mathbb{C}$ lead to the same requirements as in the real-valued case.

Our discussion shows that if we assume

$$
\operatorname{Re} \alpha>0, \quad \operatorname{Re} \delta<0, \quad p_{\min }=\frac{2|\alpha|}{|\alpha|+\operatorname{Re} \alpha}<p<\frac{2|\alpha|}{|\alpha|-\operatorname{Re} \alpha}=p_{\max },
$$

we can apply Theorem 2.8, Corollary 4.1 and Corollary 4.3 to the real-valued system (6.3), and Corollary 4.5 to the complex-valued equation (6.1). In both cases, the bound for the rate of the exponential decay reads

$$
0 \leqslant \mu \leqslant \varepsilon \frac{\nu}{p}, \quad \text { for } \nu=\frac{\sqrt{\operatorname{Re} \alpha(-\operatorname{Re} \delta)}}{|\alpha|} \text { and some } 0<\varepsilon<1,
$$

since $a_{0}=\operatorname{Re} \alpha, b_{0}=-\operatorname{Re} \delta$ and $a_{\max }=|\alpha|$. 
6.1. Spinning solitons. For the parameter values from [14], given by

$$
\alpha=\frac{1}{2}+\frac{1}{2} i, \quad \beta=\frac{5}{2}+i, \quad \gamma=-1-\frac{1}{10} i, \quad \delta=-\frac{1}{2},
$$

equation (6.1) exhibits so called spinning soliton solutions for space dimensions $d=2$ and $d=3$, see Figure 3 . The parameter values (6.10) satisfy the requirements from (6.8), and therefore our assumptions (A1)-(A11) for every $p$ with

$$
1.1716 \approx \frac{4}{2+\sqrt{2}}=p_{\min }<p<p_{\max }=\frac{4}{2-\sqrt{2}} \approx 6.8284,
$$

e.g. for $p=2,3,4,5,6$. Therefore, the solitons (and their derivatives) are exponentially localized in the sense of Theorem 2.8 and Corollary 4.1, i.e. $\mathbf{v}_{\star}$ belongs to $W_{\theta}^{2, p}\left(\mathbb{R}^{d}, \mathbb{R}^{2}\right)$ for $p \in\left(p_{\min }, p_{\max }\right)$ and for the weight function $\theta(x)=\exp \left(\mu \sqrt{|x|^{2}+1}\right)$ with exponential decay rate

$$
0 \leqslant \mu \leqslant \frac{\varepsilon}{\sqrt{2} p} .
$$

Corollary 4.1 implies that $\mathbf{v}_{\star}$ even belongs to $W_{\theta}^{k, p}\left(\mathbb{R}^{d}, \mathbb{R}^{2}\right)$ for every $k \geqslant 0$, provided $d=2, p \in\left(p_{\min }, p_{\max }\right)$ or $d=3, p \in\left[\frac{3}{2}, p_{\max }\right)$ since $\mathbf{f} \in C^{\infty}\left(\mathbb{R}^{2}, \mathbb{R}^{2}\right)$. Moreover, Corollary 4.3 shows that the solitons satisy the pointwise estimates (4.16). In Section 6.3 we will compare this with the rate of decay measured from numerical experiments.

Next, we discuss the numerical results from Figure 3 and explain how to compute the profile and velocities numerically. For all numerical computations including the eigenvalue computations we used Comsol Multiphysics 5.2, [1].

Figure $3(\mathrm{a})$ shows the spinning soliton in $\mathbb{R}^{2}$ as the solution of (6.1) on a circular disk of radius $R=20$ centered at the origin at time $t=150$. For the computation we used continuous piecewise linear finite elements with maximal stepsize $\triangle x=0.25$, the BDF method of order 2 with absolute tolerance atol $=10^{-5}$, relative tolerance rtol $=10^{-3}$ and maximal stepsize $\triangle t=0.1$, homogeneous Neumann boundary conditions and initial data

$$
u_{0}^{2 D}\left(x_{1}, x_{2}\right)=\frac{1}{5}\left(x_{1}+i x_{2}\right) \exp \left(-\frac{x_{1}^{2}+x_{2}^{2}}{49}\right), \quad x_{1}^{2}+x_{2}^{2} \leq R^{2} .
$$

Figure 3(b) shows isosurfaces of the spinning soliton in $\mathbb{R}^{3}$ obtained from the solution of $(6.1)$ on a cube $[-10,10]^{3}$ at time $t=100$. For the computation we used continuous piecewise linear finite elements with maximal stepsize $\triangle x=0.8$, the BDF method of order 2 with absolute tolerance atol $=10^{-4}$, relative tolerance rtol $=10^{-2}$ and maximal stepsize $\triangle t=0.1$, homogeneous Neumann boundary conditions and (discontinuous) initial data

$$
u_{0}^{3 D}\left(x_{1}, x_{2}, x_{3}\right)=u_{0}^{2 D}\left(x_{1}, x_{2}\right) \text { for }\left|x_{3}\right|<9 \text { and } 0 \text { otherwise. }
$$

Using these solutions as initial data, the freezing method from $[7,8]$ provides an approximate rotating wave with profile $\mathbf{w}_{\star}$ in the following format

$$
\mathbf{u}_{\star}(x, t)=\mathbf{w}_{\star}(\exp (-t S)(x-t E(t S) \tau)), \quad x \in \mathbb{R}^{d}, t \in \mathbb{R},
$$

where $S \in \mathbb{R}^{d, d}$ is skew symmetric, $\tau \in \operatorname{range}(S) \subset \mathbb{R}^{d}$ and $E$ is the analytic function $E(z)=\sum_{j=1}^{\infty} \frac{z^{j-1}}{j !}$ satisfying $E(z) z=\exp (z)-1, z \in \mathbb{C}$. In order to put 

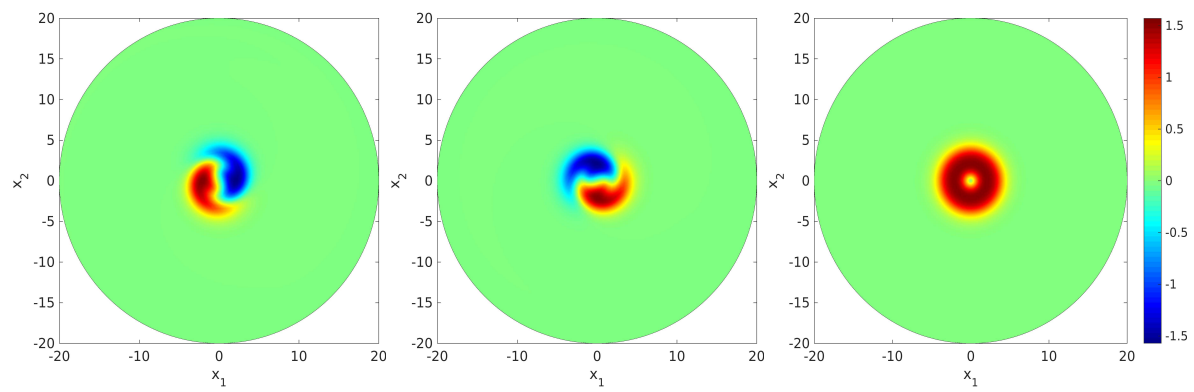

(a)
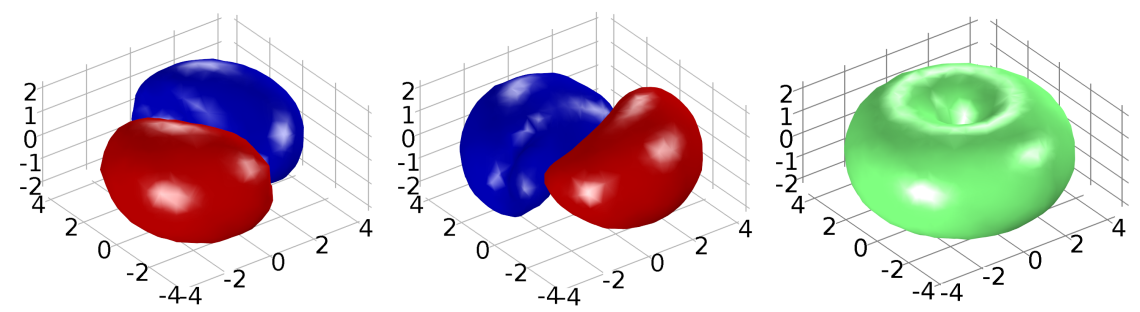

(b)

FIGURE 3. Spinning soliton of QCGL (6.1) for $d=2$ (a) and $d=3$ (b) with real part (left), imaginary part (middle) and absolute value (right). The colorbar in (a) reaches from -1.6 (blue) to 1.6 (red). The isosurfaces in (b) have values -0.5 (blue), 0.5 (red) and 0.5 (green).

this into the standard form (2.2) used in our theory, we determine the position vector $x_{\star} \in \mathbb{R}^{d}$ by solving

$$
S x_{\star}+\tau=0 .
$$

Then we may write

$$
\begin{aligned}
\exp (-t S)(x-t E(t S) \tau) & =\exp (-t S)\left(x+E(t S)(t S) x_{\star}\right) \\
& =\exp (-t S)\left(x-x_{\star}\right)+x_{\star}
\end{aligned}
$$

so that (6.13) turns into

$$
\mathbf{u}_{\star}(x, t)=\mathbf{w}_{\star}\left(\exp (-t S)\left(x-x_{\star}\right)+x_{\star}\right)=\mathbf{v}_{\star}\left(\exp (-t S)\left(x-x_{\star}\right)\right) .
$$

Thus the numerical profile $\mathbf{w}_{\star}$ is a slightly shifted version of the profile $\mathbf{v}_{\star}$ used for the theory. In practice, we solve (6.14) directly for $d=2$ and by rank-deficient least squares for $d=3$, see [26, Ex.10.8] for details. 
Using for $d=2$ the data at time $t=400$, one obtains the following values for $S_{12}, \tau$ and the center of rotation $x_{\star}^{2 D}$ of the spinning soliton

$$
\begin{aligned}
& S_{12}=1.0286, \quad \tau=\left(\begin{array}{c}
-0.0054 \\
-0.0071
\end{array}\right), \\
& x_{\star}^{2 D}:=-S^{-1} \tau=\frac{1}{S_{12}}\left(\begin{array}{c}
\tau_{2} \\
-\tau_{1}
\end{array}\right)=\left(\begin{array}{c}
-0.0069 \\
0.0052
\end{array}\right) .
\end{aligned}
$$

The rotating wave $\mathbf{u}_{\star}: \mathbb{R}^{2} \times[0, \infty) \rightarrow \mathbb{R}^{2}$ satisfies

$$
\mathbf{u}_{\star}(x, t)=\mathbf{w}_{\star}\left(e^{-t S}\left(x-x_{\star}^{2 D}\right)+x_{\star}^{2 D}\right)=\mathbf{v}_{\star}\left(e^{-t S}\left(x-x_{\star}^{2 D}\right)\right) .
$$

In case $d=3$ at time $t=500$ the rotational velocities $S_{12}, S_{13}, S_{23}$ and the translational vector $\tau$ of the spinning soliton are found to be

$$
\left(\begin{array}{l}
S_{12} \\
S_{13} \\
S_{23}
\end{array}\right)=\left(\begin{array}{c}
0.6888 \\
-0.0043 \\
-0.0043
\end{array}\right), \quad \tau=\left(\begin{array}{c}
0.0023 \\
-0.0415 \\
0.0005
\end{array}\right)
$$

The axis of rotation is $\left\{x_{\star}^{3 D}+r x_{\text {rot }}^{3 D}, r \in \mathbb{R}\right\}$ with support vector $x_{\star}^{3 D}$ and the direction $x_{\text {rot }}^{3 D}$ spanning the null space of $S$, is given by

$$
\begin{aligned}
& x_{\mathrm{rot}}^{3 D}:=\left(\begin{array}{c}
S_{23} \\
-S_{13} \\
S_{12}
\end{array}\right)=\left(\begin{array}{c}
-0.0043 \\
0.0043 \\
0.6888
\end{array}\right), \\
& x_{\star}^{3 D}:=\frac{1}{S_{12}^{2}+S_{13}^{2}+S_{23}^{2}}\left(\begin{array}{c}
S_{12} \tau_{2}+S_{13} \tau_{3} \\
-S_{12} \tau_{1}+S_{23} \tau_{3} \\
-S_{13} \tau_{1}-S_{23} \tau_{2}
\end{array}\right)=\left(\begin{array}{l}
-0.0602 \\
-0.0033 \\
-0.0004
\end{array}\right) .
\end{aligned}
$$

As above, the rotating wave $\mathbf{u}_{\star}: \mathbb{R}^{3} \times[0, \infty) \rightarrow \mathbb{R}^{2}$ satisfies

$$
\mathbf{u}_{\star}(x, t)=\mathbf{w}_{\star}\left(e^{-t S}\left(x-x_{\star}^{3 D}\right)+x_{\star}^{3 D}\right)=\mathbf{v}_{\star}\left(e^{-t S}\left(x-x_{\star}^{3 D}\right)\right) .
$$

The periods of rotation for the spinning solitons in $\mathbb{R}^{2}$ and $\mathbb{R}^{3}$ are determined by

$$
T^{2 D}=\frac{2 \pi}{\left|S_{12}\right|}=6.1085 \text { and } \quad T^{3 D}=\frac{2 \pi}{\left|\sqrt{S_{12}^{2}+S_{13}^{2}+S_{23}^{2}}\right|}=9.1216 .
$$

6.2. Spectrum and eigenfunctions at spinning solitons. Consider the eigenvalue problem for the real-valued version of the QCGL-equation

$$
\mathcal{L} \mathbf{v}(x)=\mathbf{A} \triangle \mathbf{v}(x)+\langle S x, \nabla \mathbf{v}(x)\rangle+D \mathbf{f}\left(\mathbf{v}_{\star}(x)\right) \mathbf{v}(x)=\lambda \mathbf{v}(x), x \in \mathbb{R}^{d},
$$

where $d \in\{2,3\}, \mathbf{v}: \mathbb{R}^{d} \rightarrow \mathbb{C}^{2}, \mathbf{A} \in \mathbb{R}^{2,2}$ is from (6.3), f $: \mathbb{R}^{2} \rightarrow \mathbb{R}^{2}$ from (6.4) and $S \in \mathbb{R}^{d, d}$ from (6.7).

Recall from Section 6.1 that the Ginzburg-Landau equation (6.3) exhibits spinning soliton solutions for space dimensions $d=2$ and $d=3$ and for the parameter values from (6.10).

Below we approximate solutions $(\lambda, \mathbf{v})$ of the eigenvalue problem (6.18) and apply Theorem 5.1 and Corollary 5.5 to (6.18). Instead of (6.18) we solve the eigenvalue problem

$$
\mathbf{A} \triangle \mathbf{w}(x)+\left\langle S\left(x-x_{\star}\right), \nabla \mathbf{w}(x)\right\rangle+D \mathbf{f}\left(\mathbf{w}_{\star}(x)\right) \mathbf{w}(x)=\lambda \mathbf{w}(x), x \in \mathbb{R}^{d},
$$


with $d \in\{2,3\}, S \in \mathbb{R}^{d, d}$ and $\tau \in \mathbb{R}^{d}$ from (6.16) and (6.17). Note that (6.19) is just the shifted version of (6.18) where $\mathbf{w}(x)=\mathbf{v}\left(x-x_{\star}\right), x \in \mathbb{R}^{d}$. Hence the eigenvalues are the same.

We use the following values

$$
\begin{aligned}
& \mathbf{v}_{\infty}=\left(\begin{array}{l}
0 \\
0
\end{array}\right), \quad \sigma\left(D \mathbf{f}\left(\mathbf{v}_{\infty}\right)\right)=\{\delta, \bar{\delta}\}=\left\{-\frac{1}{2}\right\}, \\
& b_{0}=-s\left(D \mathbf{f}\left(\mathbf{v}_{\infty}\right)\right)=-\operatorname{Re} \delta=\frac{1}{2} .
\end{aligned}
$$

The spectrum of $S \in \mathbb{R}^{d, d}$ is given by

$$
\begin{array}{llll}
(6.21) & d=2: & \sigma(S)=\left\{ \pm \sigma_{1} i\right\}, & \\
(6.22) & d=3: & \sigma(S)=\left\{0, \pm \sigma_{1} i\right\}, & \sigma_{1}=\sqrt{S_{12}^{2}+S_{13}^{2}+S_{23}^{2}}=0.6888 .
\end{array}
$$

For the solution of the eigenvalue problem (6.19) we use in both cases, $d=2$ and $d=3$, continuous piecewise linear finite elements with maximal stepsize $\triangle x=0.25$ (if $d=2$ ) and $\triangle x=0.8$ (if $d=3$ ), homogeneous Neumann boundary conditions and the following parameters for the eigenvalue solver

$$
\text { neigs }=800, \quad \sigma=-1, \quad \text { etol }=10^{-7},
$$

i.e. we approximate neigs $=800$ eigenvalues that are located nearest to $\sigma=-1$ and satisfy the eigenvalue tolerance etol $=10^{-7}$. As above, the profile $\mathbf{w}_{\star}$ and the pair $\left(S, x_{\star}\right)$ in $(6.19)$ are obtained from simulating the freezing system until $t=400$ for $d=2$ and until $t=500$ for $d=3$. With the data from the last time instance we then solve the eigenvalue problem (6.19).

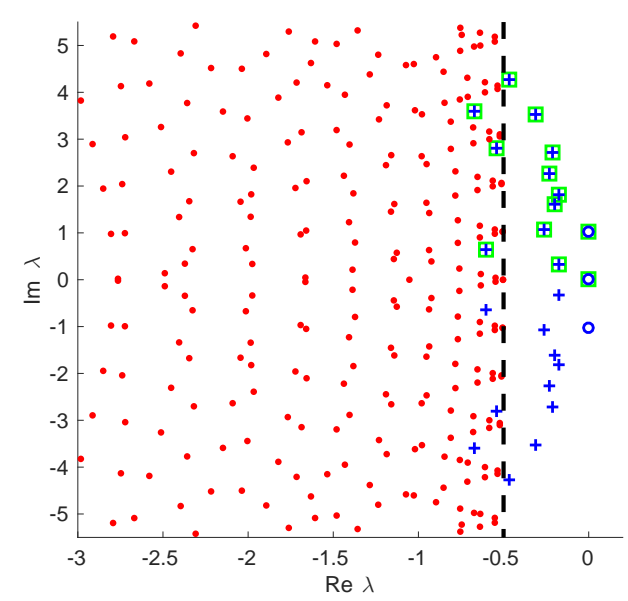

(a)

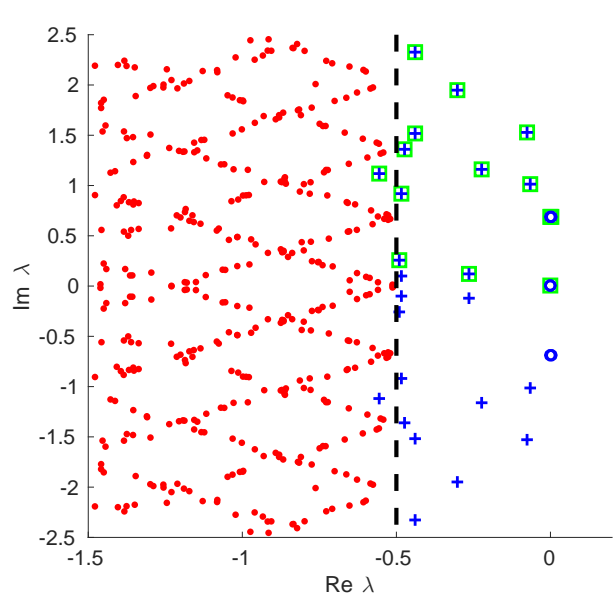

(b)

Figure 4. Numerical spectra of QCGL (6.1) linearized about a spinning soliton for $d=2(\mathrm{a})$, and $d=3(\mathrm{~b})$.

Figure 4 shows the numerical approximation $\sigma^{\text {approx }}(\mathcal{L})$ of the spectrum $\sigma(\mathcal{L})=$ $\sigma_{\text {point }}(\mathcal{L}) \cup \sigma_{\text {ess }}(\mathcal{L})$ of $\mathcal{L}$ obtained by linearizing about the spinning soliton $\mathbf{v}_{\star}$ for $d=2(\mathrm{a})$, and $d=3$ (b). Let us discuss the numerical spectra in more detail and compare them with our theoretical results: 
a) Essential spectrum: We replace $\mathbf{v}_{\star}$ in $\mathcal{L}$ by its limiting value zero and find a dispersion relation $(5.10)$, leading to

$$
\sigma_{\text {ess }}^{\text {part }}(\mathcal{L})=\left\{\lambda=-\omega^{2} \alpha_{1}+\delta_{1}+i\left(\mp \omega^{2} \alpha_{2} \pm \delta_{2}-n \sigma_{1}\right) \mid \omega \in \mathbb{R}, n \in \mathbb{Z}\right\}
$$

with $\sigma_{1}$ from (6.21) for $d=2$ and from (6.22) for $d=3$, cf. Remark 5.6 and [26, Thm.9.10]. Recall the inclusion $\sigma_{\text {ess }}^{\text {part }}(\mathcal{L}) \subseteq \sigma_{\text {ess }}(\mathcal{L})$ and taking the parameter values (6.10) into account, (6.24) reads as

$$
\sigma_{\text {ess }}^{\text {part }}(\mathcal{L})=\left\{\lambda=-\frac{1}{2}\left(\omega^{2}+1\right)+i\left(\mp \frac{1}{2} \omega^{2}-n \sigma_{1}\right) \mid \omega \in \mathbb{R}, n \in \mathbb{Z}\right\} \subseteq \sigma_{\text {ess }}(\mathcal{L}) .
$$

In both cases the part $\sigma_{\text {ess }}^{\text {part }}(\mathcal{L})$ of the essential spectrum forms a zig-zag-structure that can be considered as the union of infinitely many copies of cones. The tips of the cones $-\frac{1}{2}-i n \sigma_{1}, n \in \mathbb{Z}$ lie on the line $\delta_{1}+i \mathbb{R}=-\frac{1}{2}+i \mathbb{R}$. Therefore, the distance between two neighboring tips equals $\sigma_{1}$. The gap between the whole essential spectrum and the imaginary axis equals $b_{0}=\frac{1}{2}$, since $\operatorname{Re} \sigma_{\text {ess }}(\mathcal{L}) \leqslant-b_{0}=$ $\operatorname{Re} \delta=-\frac{1}{2}$. The inclusion $\sigma_{\text {ess }}^{\text {part }}(\mathcal{L}) \subseteq \sigma_{\text {ess }}(\mathcal{L})$ is proved in [27, Thm.9.10] for the $L^{p}$-spectrum of $\mathcal{L}$. We believe that even equality holds, i.e. $\sigma_{\mathrm{ess}}^{\text {part }}(\mathcal{L})=\sigma_{\text {ess }}(\mathcal{L})$, but this has not been proved so far.

Let us now consider the numerical results: The red dots in Figure 4 represent the approximation $\sigma_{\text {ess }}^{\text {appox }}(\mathcal{L})$ of the essential spectrum $\sigma_{\text {ess }}(\mathcal{L})$. They approximate the collection of cones in the essential spectrum. As expected, the tips are approximatively located on $-\frac{1}{2}+i \mathbb{R}$, indicated by the black dashed line. Our results show that the distance between two neighboring tips of the cones agrees with $\sigma_{1}$ from (6.21) for $d=2$ and from (6.22) for $d=3$. In particular, the approximation suggests that we have equality in (6.24). The case $d=2$ has been also treated in [5, Sec.8]. b) Point spectrum: From Theorem 5.4 and Remark 5.6 we have the relation

$$
\sigma_{\text {point }}^{\text {part }}(\mathcal{L})=\left\{0, \pm i \sigma_{1}\right\} \subseteq \sigma_{\text {point }}(\mathcal{L})
$$

with $\sigma_{1}$ from (6.21) for $d=2$ and (6.22) for $d=3$ (cf. [26, Thm.9.4] for more details). The eigenvalues $0, \pm i \sigma_{1}$ are located on the imaginary axis and have (at least) algebraic multiplicities 1 for $d=2$ and 2 for $d=3$, respectively, see Theorem 5.4. The numerical results below suggest that we do not have equality in (6.25), i.e. in general there are further isolated eigenvalues which have to be determined numerically.

Let us now consider the associated numerical results: The blue circles and the blue plus signs in Figure 4 represent an approximation $\sigma_{\text {point }}^{\text {approx }}(\mathcal{L})$ of the point spectrum $\sigma_{\text {point }}(\mathcal{L})$. The approximate eigenvalues $0, \pm i \sigma_{1}$ are visualized by blue circles. The plus signs indicate further isolated eigenvalues which belong to the point spectrum, but cannot be determined explicitly. In case $d=2$, there are 11 additional complex-conjugate pairs of isolated eigenvalues, of which 8 pairs are located to the right of the vertical black dashed line $-\frac{1}{2}+i \mathbb{R}$ and 3 pairs to the left in between the zig-zags. We emphasize that these three pairs are not numerical artifacts but are robust to spatial mesh refinement and to increase of spatial domain. Similarly, in case $d=3$, we find 12 additonal complex-conjugate pairs of isolated eigenvalues, of which 11 pairs are located to the right of the vertical line $-\frac{1}{2}+i \mathbb{R}$ and 1 pair to the left in between the zig-zags. Numerical values of the isolated eigenvalues are given in Table 1 below.

c) Eigenfunctions: The eigenfunctions associated to eigenvalues from the essential spectrum are explicitly known and bounded but never localized, i.e. they do not 


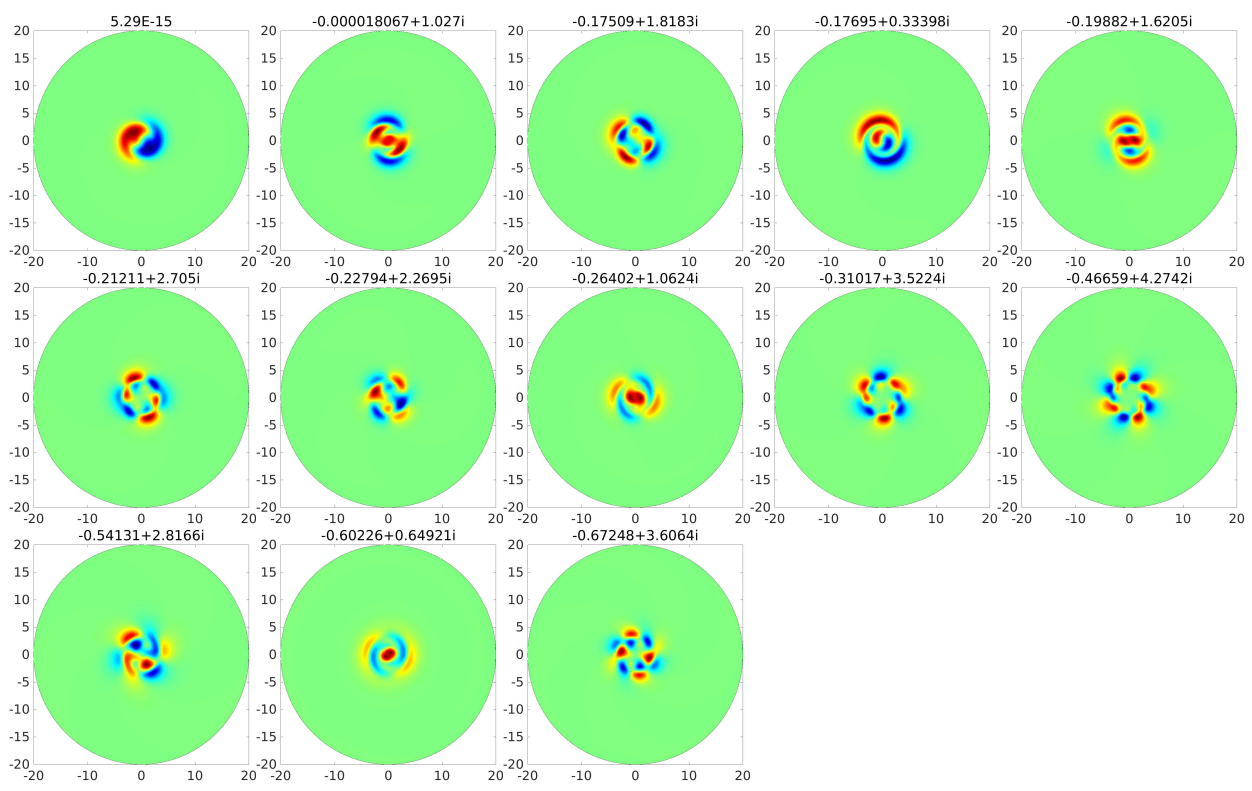

FIGURE 5. Real parts of eigenfunctions of 2D-QCGL (6.1) for a spinning soliton.

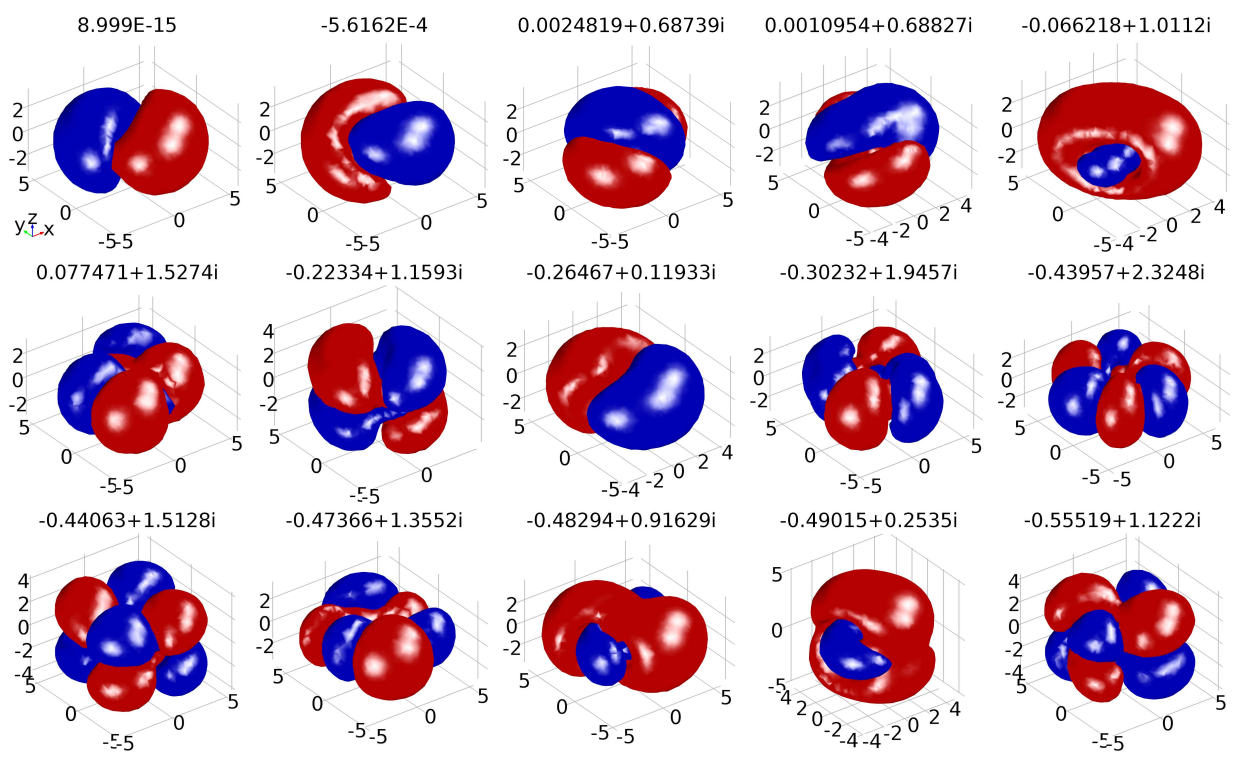

Figure 6. Isosurfaces of real parts of eigenfunctions of 3D-QCGL (6.1) for a spinning soliton.

decay in space, see [26, Thm.7.9 and 9.10]. In contrast to this, all eigenfunctions associated to eigenvalues from the point spectrum, in particular those on the imaginary axis, are exponentially localized. For eigenvalues $\lambda$ with $\operatorname{Re} \lambda>-\beta_{\infty}=-\frac{1}{2}$ this follows from our theory. To be more precise, let us introduce angular derivatives 
by

$$
D^{(1,2)}:=x_{2} D_{1}-x_{1} D_{2}, \quad D^{(1,3)}:=x_{3} D_{1}-x_{1} D_{3}, \quad D^{(2,3)}:=x_{3} D_{2}-x_{2} D_{3} .
$$

Then Theorem 5.4 yields eigenfunctions associated to eigenvalues from $\sigma_{\text {point }}^{\text {part }}(\mathcal{L})$ as follows

$$
\begin{aligned}
\lambda_{1} & =0, & & \mathbf{v}_{\mathbf{1}}=D^{(1,2)} \mathbf{v}_{\star}, \\
\lambda_{2,3} & = \pm i \sigma_{1}, & & \mathbf{v}_{\mathbf{2}, \mathbf{3}}=D_{1} \mathbf{v}_{\star} \pm i D_{2} \mathbf{v}_{\star}
\end{aligned}
$$

for $d=2$, see [26, Ex.9.6], and by

$$
\begin{aligned}
\lambda_{1}=0, & \mathbf{v}_{\mathbf{1}}=S_{12} D^{(1,2)} \mathbf{v}_{\star}+S_{13} D^{(1,3)} \mathbf{v}_{\star}+S_{23} D^{(2,3)} \mathbf{v}_{\star}, \\
\lambda_{2}=0, & \mathbf{v}_{\mathbf{2}}=S_{23} D_{1} \mathbf{v}_{\star}-S_{13} D_{2} \mathbf{v}_{\star}+S_{12} D_{3} \mathbf{v}_{\star}, \\
\lambda_{3,4}= \pm i \sigma_{1}, \quad \mathbf{v}_{\mathbf{3}, 4}= & \left(\sigma_{1} S_{13} \pm i S_{12} S_{23}\right) D_{1} \mathbf{v}_{\star} \\
& +\left(\sigma_{1} S_{23} \pm i S_{12} S_{13}\right) D_{2} \mathbf{v}_{\star} \\
& \pm i\left(S_{13}^{2}+S_{23}^{2}\right) D_{3} \mathbf{v}_{\star}, \\
\lambda_{5,6}= \pm i \sigma_{1}, \quad \mathbf{v}_{\mathbf{5}, \mathbf{6}}= & -\left(S_{13}^{2}+S_{23}^{2}\right) D^{(1,2)} \mathbf{v}_{\star} \\
& -\left(-S_{12} S_{13} \pm i \sigma_{1} S_{23}\right) D^{(1,3)} \mathbf{v}_{\star} \\
& +\left(S_{12} S_{23} \pm i \sigma_{1} S_{13}\right) D^{(2,3)} \mathbf{v}_{\star}
\end{aligned}
$$

for $d=3$, see [26, Ex.9.7]. We next study the asymptotic behavior of eigenfunctions with eigenvalues in $\sigma_{\text {point }}(\mathcal{L})$. As shown in the previous section, Theorem 5.1 and Corollary 5.5 imply that all eigenfunctions with eigenvalues $\operatorname{Re} \lambda>-\frac{1}{2}$ are exponentially localized, in the $L^{p}$ - and in the pointwise sense. The maximal exponential rate of decay for the eigenfunctions will depend on $\lambda$ as we will see in Section 6.3 below. Note that Theorem 5.1 does not apply to eigenvalues satisfying $\operatorname{Re} \lambda \leqslant-\frac{1}{2}$.

Let us now discuss the numerical results: In Figure 4, there are some isolated eigenvalues labeled by a green square. Their eigenfunctions are visualized in Figure 5 for $d=2$ and in Figure 6 for $d=3$. Both pictures show the real parts of the first component of the associated eigenfunction $\mathbf{w}: \mathbb{R}^{d} \rightarrow \mathbb{C}^{2}$. The first two eigenfunctions in Figure 5 are approximations of $\mathbf{v}_{\mathbf{1}}, \mathbf{v}_{\mathbf{2}}$ from (6.26). Their corresponding eigenvalues approximate $\lambda_{1}, \lambda_{2}$ from (6.26), as specified in the title of the figure. Similarly, the first four eigenfunctions in Figure 6 approximate $\mathbf{v}_{\mathbf{1}}, \mathbf{v}_{\mathbf{2}}, \mathbf{v}_{\mathbf{3}}, \mathbf{v}_{\mathbf{5}}$ from (6.27). Their associated eigenvalues are approximations of $\lambda_{1}, \lambda_{2}, \lambda_{3}, \lambda_{5}$ from (6.27) and again specified in the title. Note that the first eigenfunction in Figure 5 and in Figure 6 agrees with a slightly shifted version of the rotational term $\mathbf{v}_{\mathbf{1}}(x)=\left\langle S x, \nabla \mathbf{v}_{\star}(x)\right\rangle$ which arises in the rotating wave equation (1.5). The eigenfunctions $3-10$ from Figure 5 and $5-14$ from Figure 6 belong to the eigenvalues in green boxes carrying a plus sign and satisfying $\operatorname{Re} \lambda>-\frac{1}{2}$. They are ordered with decaying real parts. All eigenfunctions with eigenvalues satisfying $\operatorname{Re} \lambda>-\frac{1}{2}$ seem to decay exponentially, as expected by Theorem 5.1. The last three eigenfunctions in Figure 5 and the last eigenfunction in Figure 6 show those eigenfunctions whose eigenvalues are marked by a green box but satisfy $\operatorname{Re} \lambda \leqslant-\frac{1}{2}$. In this case Theorem 5.1 is not applicable. However, even these eigenfunctions seem to have exponential decay in space. Finally, we note that we found further isolated eigenvalues inside the zig-zag structure, see Figure 4(b), the eigenfunctions of which seem to decay exponentially in space as well. 
6.3. Rate of exponential decay for spinning solitons and their eigenfunctions. Let us consider the rates of exponential decay for spinning solitons and their associated eigenfunctions in more detail. For this purpose we compare theoretical decay rates (short: TDR), guaranteed by Theorem 2.8 and 5.1, with numerical decay rates (short: NDR) computed from our numerical results by linear regression.

a) Decay rates of spinning solitons: The maximal rate of exponential decay for the profiles of the spinning solitons, which one obtains from Corollary 4.3, is given by, cf. (6.9), (6.6),

$$
0 \leqslant \mu \leqslant \frac{\varepsilon \nu}{p}<\frac{\nu}{p}=: \mu^{\text {pro }}(p)<\frac{\nu}{\max \left\{p_{\min }, \frac{d}{2}\right\}}=: \mu_{\max }^{\text {pro }} .
$$

Taking the parameter values (6.10) into account, (6.28) implies the following upper bounds for the theoretical decay rates

$$
\mu^{\text {pro }}(p)=\frac{1}{\sqrt{2} p} \approx \frac{0.7071}{p}, \quad \mu_{\max }^{\text {pro }}= \begin{cases}\frac{\sqrt{2}+1}{4} \approx 0.6036 & , \mathrm{~d}=2 \\ \frac{\sqrt{2}}{3} \approx 0.4714 & , \mathrm{~d}=3 .\end{cases}
$$

We compare this with the numerical exponential decay rates for the profile:

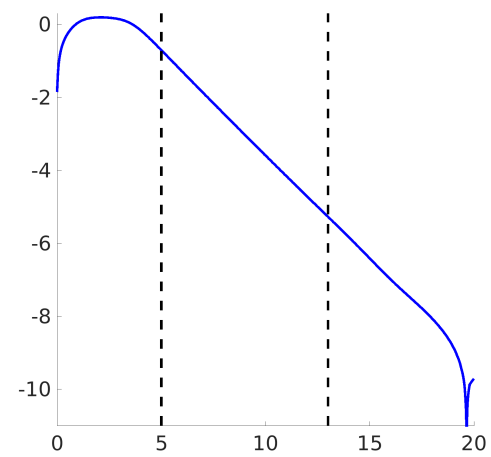

(a)

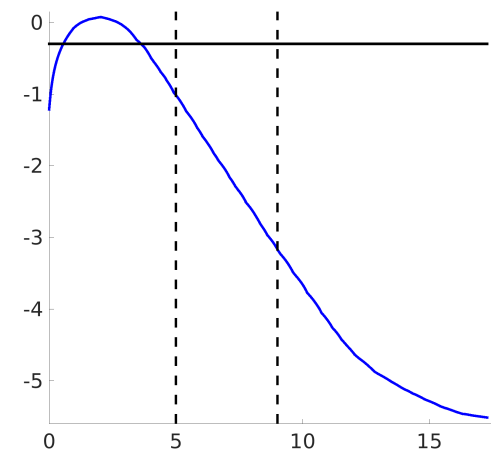

(b)

Figure 7. Numerical exponential decay rate of the spinning soliton profiles for $d=2$ (a) and $d=3$ (b) arising in the QCGL (6.3). The black line indicates the level 0.5 use for the isosurfaces in Figure $3(\mathrm{~b})$.

Figure 7 shows the absolute value of the spinning soliton profile along a straight line in radial direction, for $d=2$ in (a) and $d=3$ in (b). To be more precise, Figure 7 (a) shows the function

$$
[0,20] \rightarrow \mathbb{R}, \quad r \mapsto \log _{10}\left|\mathbf{w}_{\star}\left(r \cos \frac{\pi}{2}, r \sin \frac{\pi}{2}\right)\right|
$$

in case of $d=2$. Similarly, Figure 7 (b) shows the function

$$
[0,10 \sqrt{3}] \rightarrow \mathbb{R}, \quad r \mapsto \log _{10}\left|\mathbf{w}_{\star}\left(\frac{r}{\sqrt{3}}, \frac{r}{\sqrt{3}}, \frac{r}{\sqrt{3}}\right)\right|
$$

in case of $d=3$. The functions are almost linear at least in the regions enclosed by the black dashed lines, which are $[5,13]$ for $d=2$ and $[5,9]$ for $d=3$. In case $d=2$ the observed NDR is slightly below the TDR. This is attributed to the fact that the NDR is affected by the size of the bounded domain and by the choice of 
boundary conditions. Summarizing, this indicates that the heat kernel estimates from $[\mathbf{2 6}, \mathbf{2 7}$, which form the origin of these decay rates, are quite accurate.

b) Decay rates of eigenfunctions: The maximal rate of exponential decay for the eigenfunctions, obtained from Theorem 5.1, will now depend on $\lambda$, since

$$
\operatorname{Re} \lambda \geqslant-(1-\varepsilon) \beta_{\infty}=-(1-\varepsilon)(-\operatorname{Re} \delta) \quad \Longleftrightarrow \quad \varepsilon \leqslant \frac{\operatorname{Re} \lambda-\operatorname{Re} \delta}{-\operatorname{Re} \delta}=: \varepsilon(\lambda) .
$$

This gives us the bounds

$$
0 \leqslant \frac{\varepsilon \nu}{p} \leqslant \frac{\varepsilon(\lambda) \nu}{p}=: \mu^{\mathrm{eig}}(p, \lambda)<\frac{\varepsilon(\lambda) \nu}{\max \left\{p_{\min }, \frac{d}{2}\right\}}=: \mu_{\max }^{\mathrm{eig}}(\lambda) .
$$

With parameter values (6.10) the bounds (6.31) lead to

$$
\mu^{\mathrm{eig}}(p, \lambda)=\frac{2\left(\operatorname{Re} \lambda+\frac{1}{2}\right)}{\sqrt{2} p}, \quad \mu_{\max }^{\mathrm{eig}}(\lambda)=\frac{\sqrt{2}+1}{2}\left(\operatorname{Re} \lambda+\frac{1}{2}\right) .
$$

This shows, that the decay rate is maximal for eigenvalues on the imaginary axis and decreases linearly to 0 as $\operatorname{Re} \lambda$ approaches $-\frac{1}{2}$, cf. Figure 4 . Recall, that Theorem 5.1 does not apply for $\operatorname{Re} \lambda \leqslant-\frac{1}{2}$. For the isolated eigenvalues labeled by a green square in Figure 4, the TDR's $\mu_{\max }^{\mathrm{eig}}(\lambda)$ of the associated eigenfunctions are given in the third columns of Table 1 . We compare with the numerical exponential decay rates for the eigenfunctions:

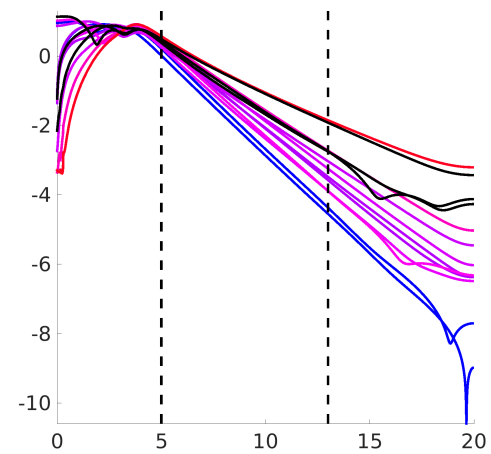

(a)

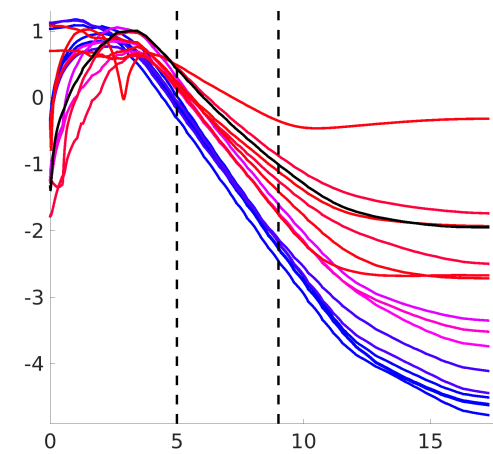

(b)

FiguRE 8. Numerical rate of exponential decay of the eigenfunctions for $d=2$ (a) and $d=3$ (b) of (6.19) linearized at a spinning soliton.

Figure 8 shows the absolute value of the eigenfunctions along the lines from (6.29) and (6.30) with $\mathbf{w}$ instead of $\mathbf{w}_{\star}$. The eigenfunctions are associated to the eigenvalues in green boxes in Figure 4. The color of the graphs vary with $\operatorname{Re} \lambda$ of the associated eigenvalue. Varying $\operatorname{Re} \lambda$ from 0 to $-\frac{1}{2}$, the graphs change color from blue to red. A red graph indicates that $\operatorname{Re} \lambda$ is near $-\frac{1}{2}$ and that the TDR is small. Finally, a black graph indicates an eigenvalue $\operatorname{Re} \lambda \leqslant-\frac{1}{2}$, in which case we do not have a TDR. All eigenfunctions are approximately linear in the regions enclosed by the black dashed lines, which are again $[5,13]$ for $d=2$, and $[5,9]$ for $d=3$. Moreover, we observe that the decay rate of the eigenfunctions decreases when the 
eigenvalue moves to the left of the imaginary axis. We note that even those eigenfunctions the eigenvalues of which satisfy $\operatorname{Re} \lambda \leqslant-\frac{1}{2}$, have exponential decay. Once more, we used linear regression on 1000 radially equispaced points to estimate the NDR. The numerical values are collected in the second columns of Table 1. Again the TDR's are surprisingly close to the NDR's with difference increasing towards $\operatorname{Re} \lambda=-\frac{1}{2}$.

\begin{tabular}{|r|c|c|}
\hline eigenvalue & NDR & TDR \\
\hline $5.29 \cdot 10^{-15}$ & 0.5713 & 0.6036 \\
$-0.00002 \pm 1.0270 i$ & 0.5730 & 0.6035 \\
$-0.17509 \pm 1.8183 i$ & 0.5001 & 0.3922 \\
$-0.17695 \pm 0.3340 i$ & 0.4815 & 0.3900 \\
$-0.19882 \pm 1.6205 i$ & 0.4139 & 0.3636 \\
$-0.21211 \pm 2.7050 i$ & 0.4652 & 0.3475 \\
$-0.22794 \pm 2.2695 i$ & 0.5155 & 0.3284 \\
$-0.26402 \pm 1.0624 i$ & 0.5355 & 0.2849 \\
$-0.31017 \pm 3.5224 i$ & 0.4044 & 0.2291 \\
$-0.46659 \pm 4.2742 i$ & 0.2984 & 0.0403 \\
$-0.54131 \pm 2.8166 i$ & 0.2972 & - \\
$-0.60226 \pm 0.6492 i$ & 0.3982 & - \\
$-0.67248 \pm 3.6064 i$ & 0.3889 & - \\
\hline
\end{tabular}

\begin{tabular}{|r|c|c|}
\hline eigenvalue & NDR & TDR \\
\hline $8.999 \cdot 10^{-15}$ & 0.5387 & 0.4714 \\
$-5.6162 \cdot 10^{-4}$ & 0.5478 & 0.4714 \\
$0.00110 \pm 0.68827 i$ & 0.5507 & 0.4714 \\
$0.00248 \pm 0.6874 i$ & 0.5398 & 0.4714 \\
$-0.06622 \pm 1.0112 i$ & 0.4899 & 0.4090 \\
$-0.07747 \pm 1.5274 i$ & 0.5355 & 0.3984 \\
$-0.22334 \pm 1.1593 i$ & 0.4756 & 0.2608 \\
$-0.26467 \pm 0.1193 i$ & 0.4785 & 0.2219 \\
$-0.30232 \pm 1.9457 i$ & 0.4649 & 0.1864 \\
$-0.43957 \pm 2.3248 i$ & 0.3595 & 0.0570 \\
$-0.44063 \pm 1.5128 i$ & 0.3310 & 0.0560 \\
$-0.47366 \pm 1.3552 i$ & 0.4781 & 0.0248 \\
$-0.48294 \pm 0.9163 i$ & 0.4145 & 0.0161 \\
$-0.48506 \pm 0.0991 i$ & 0.2126 & 0.0141 \\
$-0.49015 \pm 0.2535 i$ & 0.3307 & 0.0093 \\
$-0.55519 \pm 1.1222 i$ & 0.3581 & - \\
\hline
\end{tabular}

TABLE 1. Numerical (NDR) and theoretical (TDR) exponential decay rates of QCGL (6.1) for the eigenfunctions of the linearization at a spinning soliton for $d=2$ (left) and $d=3$ (right).

\section{References}

[1] Comsol Multiphysics 5.2, http://www.comsol.com, 2015.

[2] R. A. Adams. Sobolev Spaces, volume 65 of Pure and applied mathematics ; 65. Acad. Press, New York [u.a.], 1975.

[3] V. V. Afanasjev, N. Akhmediev, and J. M. Soto-Crespo. Three forms of localized solutions of the quintic complex ginzburg-landau equation. Phys. Rev. E, 53:1931-1939, Feb 1996.

[4] H. W. Alt. Lineare Funktionalanalysis. Springer-Verlag Berlin Heidelberg, Berlin, Heidelberg, 2006.

[5] W.-J. Beyn and J. Lorenz. Nonlinear stability of rotating patterns. Dyn. Partial Differ. Equ., $5(4): 349-400,2008$.

[6] W.-J. Beyn and J. Lorenz. Rotating Patterns on Finite Disks. unpublished, 2014.

[7] W.-J. Beyn, D. Otten, and J. Rottmann-Matthes. Stability and Computation of Dynamic Patterns in PDEs. In Current Challenges in Stability Issues for Numerical Differential Equations, Lecture Notes in Mathematics, pages 89-172. Springer International Publishing, 2014.

[8] W.-J. Beyn and V. Thümmler. Freezing solutions of equivariant evolution equations. SIAM J. Appl. Dyn. Syst., 3(2):85-116 (electronic), 2004.

[9] W.-J. Beyn and V. Thümmler. Phase conditions, symmetries and PDE continuation. In $N u$ merical continuation methods for dynamical systems, Underst. Complex Syst., pages 301-330. Springer, Dordrecht, 2007.

[10] W.-J. Beyn and V. Thümmler. Dynamics of patterns in nonlinear equivariant PDEs. GAMMMitt., 32(1):7-25, 2009.

[11] A. Cialdea and V. Maz'ya. Criterion for the $L^{p}$-dissipativity of second order differential operators with complex coefficients. J. Math. Pures Appl. (9), 84(8):1067-1100, 2005. 
[12] A. Cialdea and V. Maz'ya. Criteria for the $L^{p}$-dissipativity of systems of second order differential equations. Ric. Mat., 55(2):233-265, 2006.

[13] L.-C. Crasovan, B. A. Malomed, and D. Mihalache. Stable vortex solitons in the twodimensional ginzburg-landau equation. Phys. Rev. E, 63:016605, Dec 2000.

[14] L.-C. Crasovan, B. A. Malomed, and D. Mihalache. Spinning solitons in cubic-quintic nonlinear media. Pramana-journal of Physics, 57:1041-1059, 2001.

[15] K.-J. Engel and R. Nagel. One-parameter semigroups for linear evolution equations, volume 194 of Graduate Texts in Mathematics. Springer-Verlag, New York, 2000. With contributions by S. Brendle, M. Campiti, T. Hahn, G. Metafune, G. Nickel, D. Pallara, C. Perazzoli, A. Rhandi, S. Romanelli and R. Schnaubelt.

[16] B. Fiedler and A. Scheel. Spatio-temporal dynamics of reaction-diffusion patterns. In Trends in nonlinear analysis, pages 23-152. Springer, Berlin, 2003.

[17] K. Gustafson. The angle of an operator and positive operator products. Bull. Amer. Math. Soc., 74:488-492, 1968.

[18] K. Gustafson. Antieigenvalue analysis : with applications to numerical analysis, wavelets, statistics, quantum mechanics, finance and optimization. World Scientific, Hackensack, NJ [u.a.], 2012.

[19] L. D. Landau and V. L. Ginzburg. On the theory of superconductivity. Journal of Experimental and Theoretical Physics (USSR), 20:1064, 1950.

[20] G. Metafune, D. Pallara, and V. Vespri. $L^{p}$-estimates for a class of elliptic operators with unbounded coefficients in $\mathbf{R}^{N}$. Houston J. Math., 31(2):605-620 (electronic), 2005.

[21] A. Mielke. The Ginzburg-Landau equation in its role as a modulation equation. In Handbook of dynamical systems, Vol. 2, pages 759-834. North-Holland, Amsterdam, 2002.

[22] D. Mihalache, D. Mazilu, L.-C. Crasovan, B. A. Malomed, and F. Lederer. Three-dimensional spinning solitons in the cubic-quintic nonlinear medium. Phys. Rev. E, 61:7142-7145, Jun 2000.

[23] J. D. Moores. On the ginzburg-landau laser mode-locking model with fifth-order saturable absorber term. Optics Communications, 96(13):65-70, 1993.

[24] S. M. Nikol'skij. Approximation of Functions of Several Variables and Imbedding Theorems, volume 205 of Die Grundlehren der mathematischen Wissenschaften ; 205. Springer, Berlin [u.a.], 1975.

[25] F. W. J. Olver, D. W. Lozier, R. F. Boisvert, and C. W. Clark, editors. NIST handbook of mathematical functions. U.S. Department of Commerce National Institute of Standards and Technology, Washington, DC, 2010. With 1 CD-ROM (Windows, Macintosh and UNIX).

[26] D. Otten. Spatial decay and spectral properties of rotating waves in parabolic systems. $\mathrm{PhD}$ thesis, Bielefeld University, 2014, www.math.uni-bielefeld.de/ dotten/files/diss/Diss_ DennyOtten.pdf. Shaker Verlag, Aachen.

[27] D. Otten. Exponentially weighted resolvent estimates for complex Ornstein-Uhlenbeck systems. J. Evol. Equ., 15(4):753-799, 2015.

[28] D. Otten. A new $L^{p}$-Antieigenvalue Condition for Ornstein-Uhlenbeck Operators. Preprint, http://arxiv.org/abs/1510.00864, 2015 (submitted).

[29] D. Otten. The Identification Problem for complex-valued Ornstein-Uhlenbeck Operators in $L^{p}\left(\mathbb{R}^{d}, \mathbb{C}^{N}\right)$. Semigroup Forum, http://dx.doi.org/10.1007/s00233-016-9804-y, pages 1$38,2016$.

[30] N. Rosanov, S. Fedorov, and A. Shatsev. Motion of clusters of weakly coupled two-dimensional cavity solitons. Journal of Experimental and Theoretical Physics, 102:547-555, 2006.

[31] B. Sandstede and A. Scheel. Absolute versus convective instability of spiral waves. Phys. Rev. E (3), 62(6, part A):7708-7714, 2000.

[32] B. Sandstede and A. Scheel. Superspiral structures of meandering and drifting spiral waves. Phys. Rev. Lett., 86:171-174, Jan 2001.

[33] J. Soto-Crespo, N. Akhmediev, C. Mejía-Cortés, and N. Devine. Dissipative ring solitons with vorticity. Opt. Express, 17(6):4236-4250, Mar 2009.

[34] J. M. Soto-Crespo, N. Akhmediev, and A. Ankiewicz. Pulsating, creeping, and erupting solitons in dissipative systems. Phys. Rev. Lett., 85:2937-2940, Oct 2000.

[35] T. Tao. Real analysis. Lecture Notes, https://terrytao.wordpress.com/2009/04/30/ 245c-notes-4-sobolev-spaces/, 2009.

[36] O. Thual and S. Fauve. Localized structures generated by subcritical instabilities. J. Phys. France, 49(11):1829-1833, 1988. 
[37] V. Thümmler. Numerical bifurcation analysis of relative equilibria with Femlab. in Proceedings of the COMSOL Users Conference (Comsol Anwenderkonferenz), Frankfurt, Femlab GmbH, Goettingen, Germany, 2006.

[38] S. Trillo and W. Torruellas. Spatial Solitons. Springer Series in Optical Sciences. Springer, 2010 .

[39] W. van Saarloos and P. C. Hohenberg. Fronts, pulses, sources and sinks in generalized complex Ginzburg-Landau equations. Phys. D, 56(4):303-367, 1992.

[40] S. Zelik and A. Mielke. Multi-pulse evolution and space-time chaos in dissipative systems. Mem. Amer. Math. Soc., 198(925):vi+97, 2009.

[41] W. P. Ziemer. Weakly differentiable functions, volume 120 of Graduate Texts in Mathematics. Springer-Verlag, New York, 1989. Sobolev spaces and functions of bounded variation.

Department of Mathematics, Bielefeld University, 33501 Bielefeld, Germany

E-mail address: beyn@math.uni-bielefeld.de

URL: http://www.math.uni-bielefeld.de/〜beyn/AG_Numerik/

Department of Mathematics, Bielefeld University, 33501 Bielefeld, Germany

E-mail address: dotten@math.uni-bielefeld.de

$U R L:$ http://www.math.uni-bielefeld.de/ dotten/ 